\title{
Dysphagia Research Society 29th Anniversary Annual Meeting March 9-12, 2021
}

\section{President's Message}

Dear Colleagues:

The 29th Annual Meeting of the Dysphagia Research Society was held virtually on March 9-12, 2021. Our meeting was an outstanding success! Julie Barkmeier-Kraemer, conference co-chair, and I owe that success to all of you who took time away from your busy schedules to present your work and attend the conference. Many of you told me that the meeting was a refreshing break from pandemicrelated isolation and helped to stimulate scientific ideas in the area of swallowing and swallowing disorders.

While we could not be together this year, our virtual meeting was informative and inspiring. Thank you so much to the program committee (Jacqui Allen, Arash Babaei, Laura Baijens, Michelle Ciucci, Margaret Fynes, Kate Hutcheson, Sudarshan Jadcherla, Georgia Malandraki, Koichiro Matsuo, Anna Miles, Ashli O'Rourke, Emily Plowman, Jo Puntil, Nicole Rogus-Pulia, Allison Schaser, Catriona Steele, Rinki Desai, Emily Zimmerman) for planning such a stellar meeting. Julie and I also owe a great deal of thanks to our immediate Past President Susan Langmore. Many of her ideas for the cancelled 28th annual meeting were carried over into the 29th annual meeting. Thank you also to the DRS Executive Committee (Susan Langmore, Jacqui Allen, Georgia Malandraki)—what a great group of people work with - additional members who made up the Board of Directors (Nicole Rogus-Pulia, Catriona Steele, Martin Brodsky, Ashli O'Rourke, Johanna Kalf, Bronwyn Jones, Barbara Sonies, Cara Donohue) - and those others who chaired our committees (Catriona Steele, Julie Barkmeier-Kraemer, Ashli O'Rourke, Kendrea Garand, Michelle Troche, Sudarshan Jadcherla, Anna Miles, Rinki Desai, Joan Arvedson). We also could not have had such a successful meeting without our management staff at Badger Bay who kicked into high gear and moved mountains to make this all happen. We had a complex, ambitious meeting with many moving parts and they carried it off very well.

We brought the world together this year through the internet-we had a record number of registrants for the meeting at 622 , with global representation from 27 countries. Our vision was a meeting with highly engaging content that maximized interaction and scientific exchange. To do this, our virtual meeting environment was designed to allow you to establish profiles, upload photos, visit exhibits, attend poster sessions, watch oral presentations, engage in live questions/ answer sessions, leave comments, set up your own small meetings, and many other valuable features. We know that you made use of these functions, and this created a very dynamic experience. Some examples from our meeting metrics were the sharing of 250 photos, 1803 messages on our Community Board, 38 discussion topics, 101 participants in 6 small meetings, and over 500, 000 sponsor impressions (when an attendee clicked either on the sponsor banner or navigated to the sponsors customized resources). Meeting impact on social media was also impressive, with almost 6000 people reached on Facebook, over 700 likes on Instagram, and almost 22, 000 impressions on Twitter. For Julie and me, our favorite things were the small group discussions in the poster sessions, the rich question/answer time, the new faces from our young investigators, the diversity of speakers, topics, perspectives, and scientific orientations. We are sure that you share these favorites and have other observations to add to this list. The meeting abstracts published in this issue of Dysphagia provide an important resource to all who study and treat dysphagia.

The DRS has had many noteworthy accomplishments in 2020-2021, including our focus on diversity, equity, and inclusion; our increased social media presence to promote awareness of dysphagia; resources developed by the COVID-19 Taskforce, including our first-ever DRS publication in Dysphagia (Miles et al. 2021, 36(2):170-182); increases in our membership numbers; revision in membership categories to reflect more equity between researchers and clinicians; an improved evaluation process for our strategic plan; improvements in standardizing our awards program; a much-needed major revision of our bylaws; and new standing committees: Mentorship and Career Development and Finance. An accomplishment this year that is important to highlight is the establishment of the DRS Institute for Education (DRSIE) under the leadership of Sudarshan Jadcherla, along with receipt of external grants to allow development of the DRSIE's mission. The DRSIE's goal is to serve as a catalyst to advance the Society's research and educational mission and to provide new ideas and initiatives to enhance the society's growth.

I was privileged to be your president this year and leave the DRS in the capable hands of Jacqui Allen, DRS President for 2021-2022. I know that this year's Executive Committee, with the addition of Mark Nicosia as Secretary/Treasurer and Georgia Malandraki as President- 
Elect, will take the Society to greater heights with greater accomplishments. I look forward to seeing what the future brings in the area of greater diversity, equity, and inclusion, in international partnerships with dysphagia societies around the world, and with a larger number of quality educational opportunities through the DRS Institute for Education. Look for webinars and a fall conference coming soon. I look forward to seeing all of you at the 30th meeting to be held in Puerto Rico!

\section{Oral Presentations}

\section{Taste Stimulation and Brain Activity: A Mechanism for Neuroplastic Change?}

\section{$\underline{\text { Angela M. Dietsch }}^{1,}{ }^{2}$, Ross M. Westemeyer ${ }^{1}$, Douglas H. Schultz}

Purpose: Taste stimulation has been posited as a potential therapeutic tool for enhancing neuroplasticity and dysphagia recovery, but evidence has been confounded by inconsistencies across stimulus properties. To address this gap, we examined brain activity associated with precisely formulated and dosed taste stimuli while also accounting for participants' genetic taster status (GTS), which we previously reported as a significant modulator of swallowing physiology and underlying neural activity across tastant types.

Method(s): Twenty-one healthy adults distributed across sexes and GTS underwent functional magnetic resonance imaging (fMRI) during taste stimulation trials. Five tastants (unflavored, sour, sweetsour, lemon, and orange suspensions) were administered in timingregulated and temperature-controlled $3 \mathrm{ml}$ doses via a customized pump/tubing system. Whole brain analysis of fMRI data assessed main effects of tastant type as well as interaction effects between tastant and GTS.

Result(s): We identified differences in activity associated with tastant type in the pre- and post-central gyri, cingulate, and insular cortices bilaterally. These represent key sensory, motor, and sensorimotor integration areas involved in swallowing. Although the sweet-sour, lemon, and orange solutions had identical concentrations of citric acid and sweetener, lemon and/or orange elicited dramatically different patterns of activation than did sour and sweet-sour trials in the majority of regions. For most areas, lemon and/or orange trials yielded reductions in BOLD signal compared to unflavored trials whereas sour and sweet-sour trials elicited increases in BOLD within that region. An interaction effect between tastant type and GTS was observed in the right superior temporal gyrus, an area previously linked to swallowing effort.

Conclusions (Including Clinical Relevance): These results suggest that specific properties within very similar taste stimuli may differentially affect neural activity in swallowing-relevant regions. The findings may help explain disparities in the previous studies of taste effects on brain activity and swallow function. Further, it provides critical foundational information for defining optimal stimuli to increase brain activity in regions, which could enhance neuroplasticity and recovery when combined with traditional motor-based dysphagia therapy.

Relevant Financial Relationships: Angela Dietsch: Has affiliations to disclose;University of Nebraska-Lincoln:Salary/Stipend:Employment I Ross Westemeyer: Has affiliations to disclose;University of Nebraska-Lincoln:Salary/Stipend:Employment I Douglas Schultz: Has affiliations to disclose;University of Nebraska-Lincoln:Salary/ Stipend:Employment.

Relevant Non-financial Relationships: Angela Dietsch: Nothing to Disclose I Ross Westemeyer: Nothing to Disclose I Douglas Schultz: Nothing to Disclose.

\section{Psychometric Validation of the Dynamic Imaging Grade of Swallowing Toxicity (DIGEST) for Use in Individuals with Amyotrophic Lateral Sclerosis}

Emily K. Plowman $^{1,8}$, Justine Dallal York ${ }^{3,8}$, Lauren DiBiase ${ }^{3,8}$, Tara Segalewitz $^{2,8}$, Kayla Croft ${ }^{2,8}$, Lawrence, Shelby ${ }^{2,8}$, Nicole E. Herndon $^{2,5}$, Katherine A. Hutcheson ${ }^{7}$, Amber Anderson ${ }^{3,8}$, Jennifer Chapin ${ }^{8}$, James Wymer ${ }^{4}$, Terrie Vasilopoulos ${ }^{6}$

Purpose: The DIGEST scale is a validated instrument to index swallowing efficiency and safety in head and neck cancer. Given the paucity of valid functional outcomes of global dysphagia, we sought to psychometrically validate the DIGEST scale in people with ALS (pALS).

Method(s): 100 pALS participated and underwent: (1) standardized videofluoroscopy, (2) Functional Oral Intake Scale (FOIS), (3) Eating Assessment Tool-10 (EAT-10) and (4) ALS Functional Rating ScaleRevised Bulbar subscale (ALSFRS-Bulbar). Duplicate blinded ratings were performed/for DIGEST and MBSImP ${ }^{\mathrm{TM}}$ scales. Weighted kappa, ANOVA's (Tukey's HSD, Welch's correction), and Chi square analyses were performed.

Result(s): Mean age was $64.4(\mathrm{SD}=10.4), 50 \%$ were male, and average ALS duration was 28.2 months $(\mathrm{SD}=22.2)$. Intra-rater reliability was excellent (kappa $=0.92-1.0$ ), as was inter-rater reliability $($ kappa $=0.94)$ for DIGEST total, efficiency, and safety scores. Criterion validity: DIGEST scores significantly discriminated levels of pharyngeal pathophysiology $\left(\operatorname{MBSImP}^{\mathrm{TM}}: F(3,96)=24.7\right.$, $p<0.0001$ ), patient-rated dysphagia (EAT-10: $\mathrm{F}(3,40)=20.9$, $p<0.0001$ ), and oral intake status (FOIS $\chi^{2}: 25.4, \mathrm{df}=3$, $p<0.001)$. Construct validity: DIGEST scores significantly discriminated levels of ALS disease bulbar severity (ALSFRS-Bulbar: $F(3,93)=20.8, p<0.0001)$. There were robust pairwise mean differences between DIGEST scores for MBSImP ${ }^{\mathrm{TM}}$, EAT-10, and ALSFRS-Bulbar scores. However, DIGEST 2 vs. 3 scores (moderate vs. severe dysphagia) were not significantly different $(p>0.05)$.

Conclusions (Including Clinical Relevance): Our data indicate that the DIGEST scale is valid and reliable to distinguish normal versus impaired swallowing, as well as between mild versus moderate or severe dysphagia. However, the DIGEST did not differentiate between moderate and severe dysphagia levels. Overall, these data support the clinical utility of this instrument in ALS as a global metric of pharyngeal swallowing function.

Relevant Financial Relationships: Emily Plowman: Has affiliations to disclose;University of Florida:Salary/Stipend:Employment;Biohaven:Consulting fee:Consulting;ALS Association:Grant:Other Activities;National Institutes of Health:Grant:Other Activities I Justine Dallal York: Has affiliations to disclose;University of Florida:Salary/Stipend:Employment I Lauren DiBiase: Has affiliations to disclose;University of Florida:Salary/Stipend:Employment । Tara Segalewitz: Nothing to Disclose I Kayla Croft: Nothing to Disclose I Shelby Lawrence: Has affiliations to disclose;University of Florida:Salary/Stipend:Employment I Nicole Herndon: Has affiliations to disclose;University of Florida- UF Health:Salary/ Stipend:Employment I Katherine Hutcheson: Has affiliations to disclose;MD Anderson Cancer Center:Salary/Stipend:Employment;MD Anderson Institutional Research Grant:Grant:Other Activities;National Cancer Institute:Grant:Other Activities;National Institute of Dental and Craniofacial Research:Grant:Other Activities;PatientCentered Outcomes Research Institute:Grant:Other Activities;Thrive Foundation:Grant:Other Activities I Amber Anderson: Has affiliations to disclose;University of Florida:Salary/Stipend:Employment I Jennifer Chapin: Has affiliations to disclose;ALS Association:Salary/ Stipend:Employment I James Wymer: Has affiliations to disclose;University of Florida:Salary/Stipend:Employment । Terrie 
Vasilopoulos: Has affiliations to disclose;University of Florida:Salary/Stipend:Employment.

Relevant Non-financial Relationships: Emily Plowman: Has a NonFinancial Disclosure Affliation;Dysphagia Research Society:Professional:Membership;American Speech-Language-Hearing Association:Professional:Membership;Northeast ALS Consortium (NEALS):Professional:Volunteer membership on advisory committee or review panels I Justine Dallal York: Has a Non-Financial Disclosure Affliation;Dysphagia

Research Society:Professional:Membership;American Speech-LanguageHearing Association:Professional:Membership I Lauren DiBiase: Has a Non-Financial Disclosure Affliation;American Speech-LanguageHearing Association:Professional:Membership I Tara Segalewitz: Has a Non-Financial Disclosure Affliation;American Speech-LanguageHearing Association:Professional:Membership I Kayla Croft: Nothing to Disclose I Shelby Lawrence: Has a Non-Financial Disclosure Affliation;American Speech-Language-Hearing Association:Professional:Membership I Nicole Herndon: Has a Non-Financial Disclosure Affliation;American Speech-Language-Hearing Association:Professional:Membership I Katherine Hutcheson: Has a Non-Financial Disclosure Affliation;American Speech-Language-Hearing Association:Professional:Membership;Dysphagia

Research Society:Professional:Membership | Amber Anderson: Has a Non-Financial Disclosure Affliation;American Speech-Language-Hearing Association:Professional:Membership I Jennifer Chapin: Has a NonFinancial Disclosure Affliation;Dysphagia Research Society:Professional:Membership;American Speech-Language-Hearing Association:Professional:Membership I James Wymer: Nothing to Disclose I Terrie Vasilopoulos: Nothing to Disclose.

\section{Application of Capsaicin to the Oropharynx Reduces Bolus Size and Improves Swallow Safety in an Infant Animal Model}

Chloe E. Edmonds ${ }^{1}$, Christopher J. Mayerl ${ }^{1}$, Francois D. Gould ${ }^{2}$, Khaled Adjerid $^{1}$, Kendall E. Steer ${ }^{1}$, Laura E. Bond ${ }^{1}$, Rebecca Z. German $^{1}$

Purpose: Insight into neural mechanisms underlying sensory stimuli is critical for using sensorimotor integration in targeted dysphagia management. One specific sensory input to swallowing is via the internal branch of the superior laryngeal nerve (iSLN), which triggers a pharyngeal swallow when the bolus reaches a threshold volume. Afferent nerve fibers associated with the iSLN express the ion channel TRPV1, which is stimulated by capsaicin. Thus, the oral application of capsaicin is a useful model to explore the role of sensory stimulation in changing swallow physiology. Using an infant animal model with an iSLN lesion, we hypothesize that capsaicin will change swallow biomechanics such that bolus size will be reduced, which will ultimately improve swallow performance and safety in neurologically compromised infants.

Method(s): We used five infant pigs as a model for full-term, neonatal infants during bottle feeding. We recorded the infant pigs using high-speed videofluoroscopy at $100 \mathrm{~Hz}$ with and without capsaicin applied to the soft palate and valleculae. Prior to recording, we surgically transected the animals' iSLN and implanted radiopaque markers into relevant anatomical structures to track three-dimensional oropharyngeal kinematics. Bolus size was quantified by measuring the area of the liquid bolus in the lateral view at the initiation of the swallow, and swallow safety was evaluated using the Infant Mammalian Penetration Aspiration Scale.

Result(s): Capsaicin application significantly altered oropharyngeal kinematics in infant pigs with an iSLN lesion. Infant pigs swallowed smaller boluses after exposure to capsaicin. Further, lesioned animals treated with capsaicin experienced fewer instances of penetration and aspiration.

Conclusions (Including Clinical Relevance): Capsaicin, operating through the ion channel TRPV1, changed swallowing physiology at multiple levels: biomechanics, bolus formation, and performance. Our results suggest that swallowing deficits caused by iSLN lesion can be counteracted by agonists that increase sensory stimulation. This work illustrates the potential role of sensory stimulation as an intervention for dysphagia, when based on an understanding the neural mechanisms underlying swallowing.

Relevant Financial Relationships: Chloe Edmonds: Has affiliations to disclose;Northeast Ohio Medical University:Salary/Stipend:Employment I Christopher Mayerl: Has affiliations to disclose;Northeast Ohio Medical University:Salary/Stipend:Employment I Francois Gould: Has affiliations to disclose;Rowan School of Osteopathic Medicine:Salary/Stipend:Employment I Khaled Adjerid: Has affiliations to disclose;Northeast Ohio Medical University:Salary/ Stipend:Employment । Kendall Steer: Has affiliations to disclose;Northeast Ohio Medical University:Salary/ Stipend:Employment I Laura Bond: Has affiliations to disclose;Northeast Ohio Medical University:Salary/ Stipend:Employment I Rebecca German: Has affiliations to disclose;Northeast Ohio Medical University:Salary/ Stipend:Employment.

Relevant Non-financial Relationships: Chloe Edmonds: Nothing to Disclose | Christopher Mayerl: Has a Non-Financial Disclosure Affliation;Dysphagia Research Society:Professional:Membership । Francois Gould: Has a Non-Financial Disclosure Affliation;Dysphagia Research Society:Professional:Membership I Khaled Adjerid: Has a Non-Financial Disclosure Affliation;Dysphagia Research Society:Professional:Membership | Kendall Steer: Nothing to Disclose | Laura Bond: Nothing to Disclose I Rebecca German: Has a NonFinancial Disclosure Affliation;Dysphagia Research Society:Professional:Membership.

\section{Respiratory-Swallow Phase Patterning Linked to Swallowing Impairment in Head and Neck Cancer patients}

\section{Theresa Hopkins-Rossabi ${ }^{1}$, Bonnie Martin-Harris ${ }^{1}$}

Purpose: Healthy adults typically swallow during exhalation at midto-low lung volume, an essential element of airway protection that decreases the risk of intrusion of food or liquid into the airway. The risk of aspiration during swallowing is heightened by discoordination between respiration and swallowing, specifically in patients with HNC. The aim of this investigation was to determine the link between frequency of optimal respiratory-swallow phase pattern, swallowing impairment, and penetration/aspiration in HNC patients. The timeline for the onset of changes in respiratory-swallow phase patterns in the HNC population is not yet well understood. Therefore, the onset of the changes in respiratory-swallow phase patterns observed in HNC patients, the percentage of optimal phase patterns, swallowing impairment, and penetration/aspiration in one group of $\mathrm{HNC}$ patients prior to (pre-treatment [tx]) and another group of HNC patients following cancer treatment (post-tx) intervention were compared.

Method(s): A standard of care Modified Barium Swallow Study (MBSS) following the MBSImP ${ }^{\circledR}$ protocol with simultaneous recording of respiratory phase and lung volume at swallow onset was completed on 49 heterogeneous, prospectively seen HNC patients with dysphagia. Oral Total (OT) and Pharyngeal Total (PT) (MBSImP) and Penetration-aspiration maximum (PAS max) scores were measured. 
Result(s): A significant negative correlation between optimal respiratory and swallow coordination and 3 swallowing impairment measures (Penetration-aspiration Scale (PAS) max, Pharyngeal Total (PT), Oral Total (OT) scores with Spearman correlation coefficients of $-0.53(p<0.001),-0.50(p<0.001)$, and $-0.43(p=0.002)$, respectively. As optimal pattern decreased, swallowing impairment, and penetration-aspiration increased. Optimal respiratory-swallow pattern was significantly decreased $(p=0.03)$ in patients after cancer treatment compared to patients before cancer treatment.

Conclusions (Including Clinical Relevance): These findings indicate aberrant respiratory-swallow phase patterns and higher lung volumes at swallow initiation may signal swallow impairment and provide both an important diagnostic indicator and support retraining respiratory-swallow coordination as a treatment intervention toward reducing swallowing impairment in patients with HNC.

Relevant Financial Relationships: Theresa Hopkins-Rossabi: Nothing to Disclose I Bonnie Martin-Harris: Has affiliations to disclose;Northwestern University:Salary/Stipend:Employment;Veteran Administration:Salary/Stipend:Employment;Northern Speech Services:Consulting fee:Teaching and speaking;Medical University of South Carolina:Royalty:Consulting.

Relevant Non-financial Relationships: Theresa Hopkins-Rossabi: Nothing to Disclose I Bonnie Martin-Harris: Nothing to Disclose.

\section{Optogenetic Stimulation of Respiratory-Related Neurons Modulates Swallow and Swallow-Breathing Coordination in the Ventral Medulla}

\section{Alyssa D. Huff ${ }^{1}$, Marlusa Karlen-Amarante ${ }^{1}$, Jan Marino Ramirez}

Purpose: Swallow and breathing are highly coordinated behaviors in which disruption of this coordination leads to aspiration pneumonia, the leading cause of death in many adult and pediatric diseases. Little is known about the centrall coordination of these two behaviors. Previous work in the ventral respiratory column (VRC) has shown activity in neurons related to swallow, breathing, and both behaviors, suggesting coordination at a central network level. Evidence in the intermediate reticular nucleus ( $\mathrm{PiCo})$ as well as Pre-Bötzinger complex (PreBötC) suggest involvement in swallow patterning. We hypothesize that swallow predominately occurs during the expiratory phase of swallow due to suppression of the swallow pattern generator during inspiration. We believe manipulation of the PreBötC will alter swallow-breathing coordination, swallow duration, and sequential pattern of swallow.

Method(s): In in vivo adult mice, the ventral surface of the brainstem was exposed to place bilateral glass fiber optics connected to a blue $(447 \mathrm{~nm})$ laser over the ventral surface in the region of PreBötC or PiCo. Optogeneticly encoded mice of inhibitory (Vgat-Cre) or excitatory (Dbx1-ERT2Cre, Vglut2-Cre) neuron populations were used and activated by light stimulation. Swallow activity was measured by monopolar suction electrodes of the hypoglossal (XII) and vagus (X) nerves as well as cervical sympathetic nerve (cSN) and bipolar electromyogram (EMG) of the submental complex and laryngeal complex. Phases of breathing were measured via diaphragm EMG and swallow was stimulated orally by injection of $0.1 \mathrm{cc}$ of $2.5 \%$ citric acid water.

Result(s): We found during light stimulation at the level of the PreBötC swallow was restricted to occur during the start of postinspiration. The sequential pattern of swallow-related muscles was altered in the presence of Dbx 1cre and Vgat stimulation. During light stimulation of ChAT-cre and Vglut2-cre at the level of PiCo, we found a probability of evoking a swallow was 0.37 and 0.33 , respectively.
Conclusions (Including Clinical Relevance): We believe that the PreBötC is important in modulating swallow-breathing coordination and swallow sequence. These findings also suggest the importance of $\mathrm{PiCo}$ in swallow production. Understanding the role the ventral medulla plays in swallow behavior will allow for future therapies targeted at the neurocircuitry of swallow.

Relevant Financial Relationships: Alyssa Huff: Nothing to Disclose | Marlusa Karlen-Amarante: Nothing to Disclose | Jan Marino Ramirez: Nothing to Disclose.

Relevant Non-financial Relationships: Alyssa Huff: Nothing to Disclose I Marlusa Karlen-Amarante: Nothing to Disclose I Jan Marino Ramirez: Nothing to Disclose.

\section{Characterizing Swallows from People with Neurodegenerative Diseases Using HRCA Signals and Temporal Swallow Measurements}

\section{Cara Donohue $^{1}$, Yassin Khalifa ${ }^{2}$, Subashan Perera ${ }^{3}$, Ervin Sejdic $^{2,4}$, James L. Coyle ${ }^{1,5}$, Shitong Mao ${ }^{1}$}

Purpose: Dysphagia is highly prevalent in patients with neurodegenerative diseases (ND) and is frequently associated with adverse events, leading to faster disease progression. Swallowing in patients with ND should be monitored closely due to the progressive disease nature, likelihood of fatigue during meals, and potential day-to-day fluctuation. Undergoing instrumental swallow evaluations regularly can be challenging for patients with ND due to multifactorial health problems, physical mobility impairments, and transportation issues. High-resolution cervical auscultation (HRCA), which uses acoustic/ vibratory signals from noninvasive sensors attached to the anterior laryngeal framework during swallowing, has potential as a dysphagiascreening method. This study aimed to 1 . investigate HRCA's ability to differentiate swallows from healthy people and people with ND and 2. compare traditionally annotated temporal swallowing measures between the two groups. We hypothesized HRCA would accurately differentiate swallows and that there would be differences in temporal swallow measurements between groups.

Method(s): We analyzed 170 thin liquid swallows (20 patients with $\mathrm{ND)}$ and 171 thin liquid swallows (51 healthy age-matched adults). Participants underwent VFSSs with concurrent HRCA. We used a linear mixed model and supervised machine-learning classifiers to classify swallows and compared temporal swallow measurements between groups using a linear mixed model.

Result(s): Twenty-two HRCA signal features were statistically significant $(p<0.05)$ for predicting swallows between groups. Using HRCA signal features, logistic regression and decision trees classified swallows with $99 \%$ accuracy, $100 \%$ sensitivity, and $99 \%$ specificity. Statistically significant differences $(p<0.05)$ were found between groups for thin by cup swallows for hyoid onset to upper esophageal sphincter (UES) opening and duration of UES opening and for thin by spoon swallows for swallow reaction time, hyoid onset to UES opening, and laryngeal vestibular closure duration.

Conclusions (Including Clinical Relevance): HRCA and signal processing/machine learning techniques effectively differentiated between swallows from healthy people and people with ND. Differences in signal features may be related to differences in temporal swallow measurements, but further research is needed to clarify the relationship between HRCA signal features and specific swallow events in patients with ND.

Relevant Financial Relationships: Cara Donohue: Has affiliations to disclose; NIH: Salary/Stipend: Employment; University of Pittsburgh: Salary/Stipend: Employment I Yassin Khalifa: Has affiliations to disclose; NSF:Salary/Stipend:Employment;University of Pittsburgh:Salary/Stipend:Employment I Subashan Perera: Has affiliations 
to disclose;University of Pittsburgh:Salary/Stipend:Employment;NIH:Salary/Stipend:Employment I Ervin Sejdic: Has affiliations to disclose;University of Pittsburgh:Salary/Stipend:Employment;NIH:Salary/Stipend:Employment;NSF:Salary/Stipend:Employment I James Coyle: Has affiliations to disclose;University of Pittsburgh:Salary/Stipend:Employment;NIH:Salary/Stipend:Employment;NSF:Salary/Stipend:Employment I Shitong Mao: Nothing to Disclose.

Relevant Non-financial Relationships: Cara Donohue: Has a NonFinancial Disclosure Affliation;Dysphagia Research Society:Professional:Board membership;Dysphagia Research Society:Professional:Membership | Yassin Khalifa: Nothing to Disclose I Subashan Perera: Nothing to Disclose I Ervin Sejdic: Nothing to Disclose I James Coyle: Has a Non-Financial Disclosure Affliation;Dysphagia Research Society:Professional:Membership I Shitong Mao: Nothing to Disclose.

\section{Identifying Respiratory-Swallow Phase Patterning and Lung Volume at Swallow Initiation Using a Wearable Tri-Axis Accelerometer}

\section{Cagla Kantarcigil ${ }^{1}$, Hany Arafa ${ }^{2,3}$, Abigail B. Nellis ${ }^{1}$, John Rogers $^{2,3}$, Shuai Xu ${ }^{2,4}$, Bonnie Martin-Harris ${ }^{1,4}$}

Purpose: Recalibrating respiratory-swallow phase patterning through respiratory-swallow training results in significant airway protection and swallowing biomechanical benefits. However, identifying patterns of chest-abdominal wall movements outside of research settings is cumbersome, as it requires inductobands and specialized, nonportable devices. Wearable accelerometers that detect acceleration and motion across multiple axes could offer a feasible alternative; however, little is known about the utility of accelerometers in monitoring chest-abdominal wall patterns during breathing and swallowing. Thus, the aim of this study was to compare respiratory signals recorded during swallow trials using a tri-axis wearable accelerometer to the gold standard respiratory inductance plethysmography (RIP) recordings.

Method(s): RIP, nasal airflow, and accelerometry signals were acquired synchronously from 10 healthy young adults ( 5 males, mean age $=24$ ). Inductobands were placed around the rib cage $(\mathrm{RC})$ and abdomen $(\mathrm{AB})$. The accelerometer was affixed on the suprasternal notch. $\mathrm{RC}$ and $\mathrm{AB}$ movements were recorded during vital capacity and Spirobag maneuvers and swallow trials with $5,10,20$, and $40 \mathrm{ml}$ thin boluses. The summed $\mathrm{RC}$ and $\mathrm{AB}$ signal (2:1 $\mathrm{RC}$ to $\mathrm{AB}$ ratio) was calibrated using the known volume excursion provided by the Spirobag to estimate lung volume in $\mathrm{ml}$. Comparisons were made based on (1) number of swallows, (2) pre- and post-swallow respiratory phase, and (3) lung volume at swallow initiation. Nonparametric analyses examined the agreement between the accelerometer and RIP.

Result(s): A total of 214 swallows were analyzed. There was high percentage of agreement ( $89 \%$ ) and a strong, positive correlation $(r=0.92, p<0.05)$ between the number of swallows detected by the accelerometer and RIP. Agreement between the sensors for respiratory-swallow phase patterning was excellent $(100 \%, k=1.00)$. A Wilcoxon signed-rank test showed no statistically significant differences between estimated lung volumes at swallow initiation $(p=0.101)$

Conclusions (Including Clinical Relevance): Our findings suggest that the wearable accelerometer sensor detects swallow trials and respiratory-swallow phase patterning with high accuracy. These results also indicate lung volume at swallow initiation can be estimated using the accelerometer. Upon further testing, this sensor could offer an alternative for monitoring physiological signals in a clinical setting or a patient's home.

Relevant Financial Relationships: Cagla Kantarcigil: Has affiliations to disclose;Northwestern University:Salary/ Stipend:Employment | Hany Arafa: Has affiliations to disclose;Northwestern University:Salary/Stipend:Employment I Abigail Nellis: Has affiliations to disclose;Northwestern University:Salary/ Stipend:Employment | John Rogers: Has affiliations to disclose; Northwestern University:Salary/ Stipend:Employment;NIH:Grant:Other Activities;US Provisional Patent: Hold Patent on equipment:Ownership;Stock/equity for a small business interest in this technology:Ownership interest(e.g., stocks, stock options, or other ownership interest excluding diversified mutual funds):Ownership I Shuai $\mathrm{Xu}$ : Has affiliations to disclose;Northwestern University:Salary/ Stipend:Employment;NIH:Grant:Other Activities;US Provisional Patent: Hold Patent on equipment:Other Activities;Stock/equity for a small business interest in this technology:Ownership interest (e.g., stocks, stock options, or other ownership interest excluding diversified mutual funds):Ownership I Bonnie Martin-Harris: Has affiliations to disclose;Northwestern University:Salary/Stipend:Employment;NIH:Grant:Other Activities;VA:Grant:Other Activities;Northern Speech Services:Royalty:Other Activities;MUSC Foundation for Research Development:Royalty:Other Activities;US Provisional Patent: Hold Patent on equipment:Other Activities.

Relevant Non-financial Relationships: Cagla Kantarcigil: Has a Non-Financial Disclosure Affliation;Dysphagia Research Society:Professional:Membership | Hany Arafa: Nothing to Disclose | Abigail Nellis: Has a Non-Financial Disclosure Affliation;Dysphagia Research Society:Professional:Membership;ASHA:Professional:Membership | John Rogers: Nothing to Disclose I Shuai Xu: Nothing to Disclose I Bonnie Martin-Harris: Has a Non-Financial Disclosure Affliation;American Speech Language and Hearing Association:Professional:Membership;ABSSD:Professional:Membership;Dysphagia Research Society:Professional:Membership.

\section{Quantifying Bolus Residue and Its Risks in Children: A Videofluoroscopic Study}

\section{$\underline{\text { Isuru Dharmarathna }}^{1,2}$, Anna Miles ${ }^{3}$, Jacqueline Allen ${ }^{3}$}

Purpose: Post-swallow residue is a clinical sign of swallow impairment and has shown strong association with aspiration. Videofluoroscopy (VFSS) is considered the gold standard to identify pharyngeal residue. However, subjective binary observation (present or absent) fails to provide important information on volume or location of residue and lacks objectivity and reproducibility. Reliable judgement of changes in residue over time and treatment is, therefore, challenging. We aimed to (i) determine the reliability of quantifying pharyngeal residue in children using the bolus clearance ratio (BCR), (ii) determine associations between $\mathrm{BCR}$ and other timing and displacement measures of oropharyngeal swallowing, and (iii) investigate the association between BCR and aspiration in children. Method(s): In this single-center retrospective observational study, VFSS data were recorded at 30 frames per second for quantitative analysis using a specialized software. We obtained a set of quantitative and descriptive VFSS measures from 553 children (0-21 years) using a standard protocol. We measured BCR in this pediatric cohort and tested for inter-rater and intra-rater reliability using a random sample of 50 infants $(<9$ months) and 116 children $(>1$ year).

Result(s): Good inter-rater (ICC $=0.86,95 \%$ CI $0.74-0.961$, $p<0.001)$ and excellent intra-rater reliability were achieved for BCR (ICC $=0.97,95 \%$ CI $0.91-1.000, p=001)$. Significant correlations between $\mathrm{BCR}$ and pharyngeal constriction ratio (PCR) and total 
pharyngeal transit time (TPT) were reported $(p<0.05)$. Using binomial logistic regression modelling, we found BCR was predictive of aspiration in children $\left(\chi^{2}(13)=58.093, p<0.001,64.9 \%\right)$. Children with BCR of $\geq 0.1$ were four times more likely to aspirate. Conclusions (Including Clinical Relevance): BCR is a reliable clinically useful measure to quantify post-swallow residue in children, which can be used to identify and treat children with swallow impairments, as well as to measure outcomes of intervention. Due to its ability to predict aspiration in children, clinicians can use its threshold score to determine likelihood of aspiration, when it is not visible on VFSS.

Relevant Financial Relationships: Isuru Dharmarathna: Nothing to Disclose I Anna Miles: Nothing to Disclose | Jacqueline Allen: Nothing to Disclose.

Relevant Non-financial Relationships: Isuru Dharmarathna: Nothing to Disclose I Anna Miles: Nothing to Disclose I Jacqueline Allen: Nothing to Disclose.

\section{Identifying the Barriers and Enablers to Implementation of the International Dysphagia Diet Standardisation Initiative (IDDSI) and Provision of Texture-Modified Diets (TMDs) in New Zealand Age- Care Facilities}

\section{Xiaojing S. Wu ${ }^{1}$, Anna Miles ${ }^{2}$, Andrea Braakhuis ${ }^{1}$}

Purpose: Texture-modified diets (TMDs) are a common intervention for older adults with swallowing difficulties to improve swallow safety, efficiency, or enjoyment. The International Dysphagia Diet Standardization Initiative (IDDSI) provides a framework for terminology, definitions, and testing of TMDs. This observational mixed methods study used the Consolidated Framework for Implementation Research (CFIR) to evaluate the effectiveness of IDDSI adoption in aged-care facilities (ACFs) and identify barriers and enablers to facilitate future implementation.

Method(s): Five New Zealand ACFs who had adopted IDDSI $>12$ months previously were recruited. Evaluation tools were developed based on CFIR constructs, integrating data from (i) TMD nutritional menu audit, (ii) TMD IDDSI audit, (iii) mealtime observations, (iv) manager interviews, and (v) staff self-administrated surveys.

Result(s): All facility and kitchen managers were IDDSI aware and had access to online resources. IDDSI compliance for TMDs was higher for Level 5: Minced \& Moist (25-75\%) than Level 4: Puree $(0-75 \%)$ or Level 6: Soft \& Bite Sized $(0-25 \%)$. Three sites had changed to commercially compliant products post-IDDSI adoption. This had cost implications. Awareness of IDDSI amongst staff ranged from 5 to $79 \%$ and $<50 \%$ of staff surveyed felt sufficiently trained. Awareness was greater in large sites and where mandated by head office. Managers agreed staff needed more training. Staff were keen to learn more about IDDSI and all believed it would improve food safety and quality of care. Staff IDDSI knowledge was $<50 \%$. Managers had not mandated auditing, and they felt this had led to reduced perceived importance. Lack of a dedicated project lead and no speech pathology onsite were perceived barriers. Collaboration between care and kitchen staff and support from allied health assisted implementation.

Conclusions (Including Clinical Relevance): ACF staff were aware of IDDSI but TMDs were not all compliant and staff knowledge was poor. Using the CFIR, barriers and enablers identified from this study can improve future implementation effectiveness. Staff should have access to regular training. Multidisciplinary collaboration is essential. ACFs should consider TMD auditing on a regular basis. Successful implementation of IDDSI allows improvement of quality of care and patient safety but requires a systematic implementation plan.

Relevant Financial Relationships: Xiaojing Wu: Nothing to Disclose I Anna Miles: Has affiliations to disclose;University of Auckland:Salary/Stipend:Employment I Andrea Braakhuis: Has affiliations to disclose;University of Auckland:Salary/ Stipend:Employment.

Relevant Non-financial Relationships: Xiaojing Wu: Nothing to Disclose । Anna Miles: Has a Non-Financial Disclosure Affliation;DRS:Professional:Membership I Andrea Braakhuis: Nothing to Disclose.

\section{Increased Viscosity Improves Swallow Outcomes in Infants Through Altering the Physiology of Tongue Function, But Not Hyoid or Soft Palate Function}

\section{Christopher J. Mayerl ${ }^{1}$, Chloe E. Edmonds ${ }^{2}$, Alexis M.Myrla ${ }^{1}$, Bethany M. Stricklen $^{1}$, Emily A. Catchpole ${ }^{1}$, Laura E. Bond ${ }^{1}$, Rebecca Z. German ${ }^{1}$}

Purpose: One of the most ubiquitous interventions for treating dysphagic patients, including infants, is to thicken liquids. The outcomes of thickening liquids are well understood and include reduced rates of aspiration and increased weight gain, although they are associated with a reduced quality of life. Yet we have little understanding of how thickened liquids change the mechanisms and physiology governing performance. Without insight into the physiologic responses to thickened liquids, interventions to treat dysphagia will continue to merely circumvent physiology, rather than to improve the underlying physiology causing dysphagia. Using an animal model to examine the impact of thickened liquids on infant feeding, we hypothesized that thickened liquids would increase swallow safety by modifying tongue movements more than hyoid or soft palate mechanics.

Method(s): We used high-speed biplanar videofluoroscopy (100fps) to document infant pigs feeding on thin (IDDSI 1) and xanthan-gumthickened (IDDSI 3) liquids. We measured bolus size and swallow safety for each swallow under both conditions. We also tracked radioopaque markers on the hyoid, posterior tongue, and soft palate in three dimensions, and measured tongue kinematics during suckling and swallowing.

Result(s): As expected, bolus size and the frequency of aspiration decreased with thickened liquids. We found that the movements of the hyoid and soft palate did not differ during swallowing between viscosities, although the posterior tongue moved more at lower viscosity. However, behaviors associated with suction generation were higher at low viscosity.

Conclusions (Including Clinical Relevance): Our results suggest that although increasing viscosity improves swallow safety and reduces bolus size in infants, it does not alter the movements of the hyoid or soft palate during the swallow. Instead, the sensorimotor feedback associated with processing thickened liquids results in changes in tongue function, which alters sucking dynamics, as well as tongue movements during swallowing. Understanding the physiologic response to increased viscosity may lead to interventions that improve swallow safety without necessitating the need to thicken liquids.

Relevant Financial Relationships: Christopher Mayerl: Nothing to Disclose | Chloe Edmonds: Nothing to Disclose | Alexis Myrla: Nothing to Disclose I Bethany Stricklen: Nothing to Disclose I Emily Catchpole: Nothing to Disclose I Laura Bond: Nothing to Disclose I Rebecca German: Nothing to Disclose.

Relevant Non-financial Relationships: Christopher Mayerl: Nothing to Disclose I Chloe Edmonds: Nothing to Disclose I Alexis Myrla: Nothing to Disclose I Bethany Stricklen: Nothing to Disclose I Emily 
Catchpole: Nothing to Disclose I Laura Bond: Nothing to Disclose | Rebecca German: Nothing to Disclose.

\section{Variability in Paediatric Swallowing: A Videofluoroscopic Analysis}

\section{Laura Fuller $^{1,2}$, Anna Miles ${ }^{1}$, Isuru Dharmarathna ${ }^{1}$, Jacqueline Allen $^{1,3}$}

Purpose: Clinicians often report swallow variability or inconsistency in infants as concerning in feeding evaluations. However, little is known about whether this represents pathological incoordination or normal physiologic variance in a developing child. Our retrospective study explored quantitative fluoroscopic swallow measures in 50 bottle-fed infants (0-9 months) with feeding concerns. Our research questions were: is there variability in pharyngeal timing and displacement in infants referred for fluoroscopy? and is variability associated with aspiration risk?

Method(s): Measures were taken from a mid-feed, 20-s loop recorded at 30 frames per second. Each swallow within the 20-s loop ( $n=349$ swallows) was analysed using quantitative digital measures of timing, displacement, and coordination using specialist software $\left(\right.$ Swallowtail $\left.{ }^{\mathrm{TM}}\right)$. Two-blinded raters measured all swallows with strong inter-rater reliability (ICC $0.78,95 \%$ CI 0.73-0.83). Swallow frequency, suck-swallow ratio, residue, and penetration-aspiration were also rated on every swallow.

Result(s): Variability in pharyngeal timing and displacement was identified across all infants but did not correlate with aspiration $(p>0.05) .16$ infants $(32 \%)$ aspirated; however, aspiration did not occur on every swallow (rate $10-50 \%$ of swallows). Swallow frequency varied from 1 to 15 within the 20 -second loops. Suckswallow ratios varied from 1:1 to $6: 1$. In infants with $>3$ sucks per swallow, there was an associated increased incidence of aspiration $(p<0.001)$

Conclusions (Including Clinical Relevance): In summary, pharyngeal timing and displacement variability are present in young children referred with feeding difficulties but does not correlate with aspiration. Suck-swallow ratio is an important risk factor for aspiration that can be observed at bedside without radiation. These objective measures provide insight into infant swallowing physiology and deserve further exploration.

Relevant Financial Relationships: Laura Fuller: Nothing to Disclose I Anna Miles: Nothing to Disclose I Isuru Dharmarathna: Nothing to Disclose I Jacqueline Allen: Nothing to Disclose.

Relevant Non-financial Relationships: Laura Fuller: Nothing to Disclose I Anna Miles: Nothing to Disclose I Isuru Dharmarathna: Nothing to Disclose I Jacqueline Allen: Nothing to Disclose.

\section{Swallowing and Gastrointestinal Deficits in the Pink1-/ - Rat Model of Parkinson Disease}

\section{$\underline{\text { Maryann N. Krasko }}^{1,2}$, Cynthia A. Kelm-Nelson², Michelle R. Ciucci $^{1,2,3}$}

Purpose: Parkinson disease (PD) is a whole-body neurodegenerative disorder. Recent evidence suggests that CNS pathology may originate in the gastrointestinal (GI) tract during the prodromal phase. Early motor and non-motor signs such as dysphagia, delayed gastric emptying, and constipation may serve as potential biomarkers; however, because they are common, non-specific, or age related, they often go unreported, making the study of PD-GI dysfunction in the early stages difficult. To overcome this limitation, early-onset genetic animal models of PD, such as the Pink1-/- rat, are a useful tool for studying swallowing and digestive deficits in this prodromal phase. We hypothesized that Pink1-/- rats would show early and progressive oropharyngeal dysphagia, delayed gastric emptying, and constipation. Method(s): Thirty male rats were tested at 4 (Pink1-/ $-=15$, wildtype $(\mathrm{WT})$ control $=15)$ and $6($ Pink $1-/-=7, \mathrm{WT}=6)$ months of age. Videofluoroscopic studies (30 fps) of rats ingesting a peanutbutter-barium mixture were used to measure mastication rate (cycles/ s) and bolus area $\left(\mathrm{mm}^{2}\right)$. A second study tracked barium contents through the stomach, small intestine, caecum, and colon at hours 0-6 post-barium gavage. Data for number and weight (g) of fecal emissions over 24-h were also collected. Two- and three-way mixed model ANOVAs were performed for swallowing and GI data, respectively $(\alpha<0.05)$.

Result(s): Compared to WTs, Pink1-/- rats showed slower mastication rate $(p<0.001)$ and larger bolus size $(p<0.001)$ at both 4 and 6 months of age. There were no differences for gastric emptying $(p>0.05)$. While WTs showed the presence of contents in the colon at hour 2, Pink1-/- rats did not; at hour 3, Pink1-/- rats had fewer contents within the colon compared to WTs $(p<0.01)$. Pink1-/also had a lower fecal pellet count and higher fecal pellet weight after $24 \mathrm{~h}$ at 6 months $(p<0.01)$.

Conclusions (Including Clinical Relevance): Results demonstrate that oropharyngeal swallowing dysfunction occurs in Pink1-/- rats as early as 4 months of age. Delayed transit into the colon and constipation-like signs are also evident in this model. The presence of these early oropharyngeal and GI deficits in Pink1-/- rats are analogous to those observed in human PD and suggests that this model of PD may be useful in studying mechanisms underlying Parkinsonian pathology to advance identification and treatment.

Relevant Financial Relationships: Maryann Krasko: Has affiliations to disclose;University of Wisconsin-Madison:Grant:Employment I Cynthia Kelm-Nelson: Has affiliations to disclose;University of Wisconsin-Madison:Salary/Stipend:Employment I Michelle Ciucci: Has affiliations to disclose;University of Wisconsin-Madison:Salary/ Stipend:Employment;NIH:Grant:Employment.

Relevant Non-financial Relationships: Maryann Krasko: Has a Non-Financial Disclosure Affliation;Dysphagia Research Society:Other relationship:Membership | Cynthia Kelm-Nelson: Nothing to Disclose I Michelle Ciucci: Has a Non-Financial Disclosure Affliation;National Foundation of Swallowing Disorders:Other relationship:Board membership;Dysphagia Research Society:Other relationship:Volunteer membership on advisory committee or review panels.

\section{Respiratory-Swallow Training and Its Effects on Swallowing Outcomes in Parkinson's Disease: A Prospective Cohort Treatment Study}

\section{James A. Curtis ${ }^{1}$, Brianna Kiefer ${ }^{1,2}$, Jessica Huber ${ }^{2}$, Sonja M.} Molfenter $^{3}$, Michelle S. Troche ${ }^{1}$

Purpose: Respiratory-swallow coordination is frequently impaired in Parkinson's disease (PD) and is associated with compromised swallowing safety and efficiency. The aims of this study were to examine the effects of four sessions of respiratory-swallow training (RST) on respiratory-swallow coordination and swallowing outcomes in PD. 
Method(s): People with PD were recruited to complete two pre-RST evaluations (1 month apart), four once-weekly sessions of RST, an immediate post-RST evaluation, and a 1-month post-RST evaluation. Respiratory phase patterning, respiratory pause duration, pharyngeal residue, and penetration-aspiration were assessed using simultaneous respiratory inductive plethysmography, nasal airflow monitoring, surface electromyography, and flexible endoscopic evaluation of swallowing. Repeated measures parametric and non-parametric statistical analyses were used to examine post-RST changes in the proportion of swallows exhibiting the exhale-swallow-exhale pattern, average respiratory pause duration, and proportion of swallows exhibiting pharyngeal residue and penetration-aspiration.

Result(s): Twenty-four participants were recruited. Due to COVID19 , only 12 completed the protocol to the point of the immediate postRST assessment. There were no differences in any of the outcome measures during the delayed intervention control phase, indicating stability in function prior to RST. Results revealed large post-RST improvements in the accuracy $(p<0.0005, d=2.72)$ and consistency $(p=0.007, r=1.55)$ of respiratory phase patterning and a large decrease in average respiratory pause duration ( $p=0.018, d=0.74)$. There were also significant decreases in the frequency of penetrationaspiration $(p<0.0005, d=0.81)$, and in the presence of residue within the valleculae $(p=0.001, d=0.94)$, piriforms $(p=0.003$, $d=1.22)$, laryngeal vestibule $(p<0.0005, d=0.72)$, and vocal folds ( $p=0.003, d=0.16)$ immediately following RST.

Conclusions (Including Clinical Relevance): RST appears to be an efficacious skill-based treatment for swallowing rehabilitation in people with PD, dysphagia, and suboptimal respiratory-swallow coordination. In this cohort, four sessions of RST led to robust improvements in respiratory-swallow coordination, pharyngeal residue, and penetration-aspiration. Future research should compare the effects of RST versus other established dysphagia interventions on swallowing outcomes in PD.

Relevant Financial Relationships: James Curtis: Has affiliations to disclose;Teachers College, Columbia University:Salary/Stipend:Employment;American Brain Foundation:Grant:Independent contractor (Including contracted research);Parkinson's Foundation:Grant:Independent contractor (Including contracted research);American Academy of Neurology:Grant:Independent contractor (Including contracted research) I Brianna Kiefer: Has affiliations to disclose;NIH:Contracted Research:Independent contractor (Including contracted research) I Jessica Huber: Has affiliations to disclose;Purdue University:Salary/Stipend:Independent contractor (Including contracted research);SpeechVive:Royalty:Ownership । Sonja Molfenter: Has affiliations to disclose;New York University:Salary/Stipend:Employment I Michelle Troche: Has affiliations to disclose;Dysphagia Research Society:Honoraria:Other Activities;Medbridge Inc.:Royalty:Other Activities;Teachers College, Columbia University:Salary/Stipend:Employment;Michael J Fox Foundation:Grant:Independent contractor (Including contracted research);National Ataxia Foundation:Grant:Independent contractor (Including contracted research).

Relevant Non-financial Relationships: James Curtis: Has a NonFinancial Disclosure Affliation;Dysphagia Research Society:Professional:Membership;ASHA:Professional:Membership;Dysphagia

Research Society:Professional: Other volunteer activities I Brianna Kiefer: Has a Non-Financial Disclosure Affliation;Dysphagia Research Society:Professional:Membership | Jessica Huber: Has a Non-Financial Disclosure Affliation;Dysphagia Research Society:Professional:Membership I Sonja Molfenter: Has a Non-Financial Disclosure Affliation;Dysphagia Research Society:Professional:Membership;Dysphagia Research Society:Professional: Other volunteer activities I Michelle Troche: Has a Non-Financial Disclosure Affliation;Dysphagia Research Society:Professional:Membership.
Understanding Barriers and Facilitators to Speech Pathology Services in the Emergency Department

\section{Pranika Lal ${ }^{1,2}$, Laurelie Wishart ${ }^{3,2}$, Elizabeth Ward ${ }^{3,2}$, Maria Schwarz $^{1}$, Marnie Seabrook ${ }^{1}$, Anne Coccetti ${ }^{1}$}

Purpose: Early detection of dysphagia can reduce associated medical comorbidities and subsequent burden of care. As most patients admitted to acute care services present first via the Emergency Department (ED), timely speech-language pathology (SLP) involvement in the ED services may improve patient outcomes and help reduce length of hospitalisation and associated service costs. To date, however, there is limited research into the models of ED SLP dysphagia services that currently exist and the factors that have influenced establishment of these services. This study utilised qualitative methodology to understand current models of SLP service provision in Australian ED settings and explore factors which have influenced establishment, implementation and sustainability.

Method(s): Semi-structured interviews were conducted with 12 clinicians working in acute hospital facilities with a SLP ED service. Interview transcripts were first analysed using plain content analysis to identify key themes. Sub-analysis using the Consolidated Framework for Implementation Research (CFIR) model was undertaken on 5 interviews which showcased facilities with established/consolidated models of care.

Result(s): Participants described a spectrum of SLP ED service models, ranging from limited/ad-hoc SLP involvement in care, to formal, dedicated models with proactive SLP-led screening procedures. Nine CFIR constructs (including innovation source, external policy and incentives, networks and communications, stakeholders and reflecting and evaluating) were identified as facilitators, while 4 constructs (adaptability, cost, compatibility, available resources) were identified as current barriers to service provision. Education and networks within ED were recognised as key factors to promote sustainability of current services. Improved screening/referral processes, assessment of clinical populations other than stroke, and increased funding resources were identified as areas requiring improvement for future services.

Conclusions (Including Clinical Relevance): Findings demonstrate the diverse and still largely emerging nature of SLP ED models of care across Australia, and key factors that have influenced service provision/implementation, as well as areas for future service optimisation. Further research is needed to optimise service models for dysphagia management within the ED setting.

Relevant Financial Relationships: Pranika Lal: Has affiliations to disclose;Queensland Health:Salary/Stipend:Employment I Laurelie Wishart: Has affiliations to disclose;Queensland Health:Salary/ Stipend:Employment | Elizabeth Ward: Has affiliations to disclose;Queensland Health:Salary/Stipend:Employment I Maria Schwarz: Has affiliations to disclose;Queensland Health:Salary/ Stipend:Employment I Marnie Seabrook: Has affiliations to disclose;Queensland Health:Salary/Stipend:Employment | Anne Coccetti: Has affiliations to disclose;Queensland Health:Salary/ Stipend:Employment.

Relevant Non-financial Relationships: Pranika Lal: Has a Non-Financial Disclosure Affliation;Speech Pathology Australia:Professional:Membership | Laurelie Wishart: Has a NonFinancial Disclosure Affliation;Speech Pathology Australia:Professional:Membership;DRS:Professional:Membership I Elizabeth Ward: Has a Non-Financial Disclosure Affliation;Speech Pathology Australia:Professional:Membership;DRS:Professional:Membership | Maria Schwarz: Nothing to Disclose I Marnie Seabrook: Nothing to Disclose I Anne Coccetti: Nothing to Disclose. 


\section{Incidence and Risk Factors for Dysphagia Following Cervical Spine Injury}

James E. Babowice ${ }^{1}$, Jenna Rimko' ${ }^{1}$, Hanna Aberra ${ }^{1}$, Linda Xu ${ }^{1}$, Jennifer Chen $^{1}$, Andrea Alonso ${ }^{1}$, Elan Jeremitsky ${ }^{1}$, Eunice Chung ${ }^{1}$

Purpose: Dysphagia following cervical spine injury (CSI) results in substantial morbidity as a result of aspiration; however, the incidence and risk factors for its development are not well described. Our objective is to define the incidence of dysphagia in patients following CSI, identify risk factors for its development, and examine its impact on patient outcomes.

Method(s): Adults with CSI admitted to a level 1 trauma center from 2016 to 2019 were identified for inclusion. Patients with a pre-existing dysphagia diagnosis were then excluded. All patients with dysphagia following CSI based on speech evaluation or with documented aspiration were identified and then compared to CSI patients without dysphagia. Associations between clinical variables and subsequent dysphagia were identified using multivariable logistic regression.

Result(s): Of 713 patients with CSI, 97 developed dysphagia (13.6\%). Age greater than 60 was a significant demographic risk factor for dysphagia (OR 1.03, $p<0.01)$. Intra-cerebral hemorrhage (OR 2.44, $p<0.01$ ), facial fracture (OR 3.43, $p<0.01$ ), and spinal cord injury (OR 2.47, $p=0.03$ ) were injury complex features associated with significant increased risk. Injuries at cervical spine levels C4-C6 (OR 1.87, $p=0.03$ ) and anterior column vertebral injuries (OR 1.94, $p<0.01$ ) were significant anatomic risk factors. Intubation following cervical injury (OR 21.5, $p<0.01$ ) and cervical spine surgery (OR 3.33, $p<0.01$ ) were interventions associated with significantly increased risk. Dysphagia following cervical spine injury was associated with significantly increased risk of mortality (OR 4.10, $p<0.01)$

Conclusions (Including Clinical Relevance): In the largest sample examining dysphagia following CSI to date, significant demographic, anatomic, injury complex, and iatrogenic risk factors for the development of dysphagia were identified. Improved understanding of risk factors for dysphagia following CSI can improve screening of at risk patients prior to an aspiration event and promote harm reduction.

Relevant Financial Relationships: James Babowice: Nothing to Disclose I Jenna Rimko: Nothing to Disclose I Hanna Aberra: Nothing to Disclose I Linda Xu: Nothing to Disclose I Jennifer Chen: Nothing to Disclose I Andrea Alonso: Nothing to Disclose I Elan Jeremitsky: Nothing to Disclose I Eunice Chung: Nothing to Disclose.

Relevant Non-financial Relationships: James Babowice: Nothing to Disclose I Jenna Rimko: Nothing to Disclose I Hanna Aberra: Nothing to Disclose I Linda Xu: Nothing to Disclose I Jennifer Chen: Nothing to Disclose I Andrea Alonso: Nothing to Disclose I Elan Jeremitsky: Nothing to Disclose I Eunice Chung: Nothing to Disclose.

\section{Airway Protection Following Unilateral DBS in Parkinson's Disease: Does Hemisphere Make a Difference?}

\section{May Smith-Sherry $^{1,2}$, Nicole Herndon ${ }^{2}$, Karen Hegland ${ }^{1,2}$}

Purpose: Research on the effects of deep-brain stimulation in both the globus pallidus internus (GPi) and the subthalamic nucleus (STN) on swallowing in Parkinson's disease (PD) has yielded inconsistent findings. The target laterality (right versus left) has not been investigated, which may explain some of the previous inconsistencies. Lesion studies in other populations (i.e. stroke) have shown that lesions in the right cerebral hemisphere may be more highly associated with swallowing impairment compared to left-hemispheric lesions. Therefore, the aim of this study was to examine the effect of laterality of single-lead implants (either GPi or STN) on airway protection in $\mathrm{PD}$.

Method(s): A retrospective data analysis was conducted on patients with PD $(N=85)$ who underwent unilateral DBS (left 65\%) in either the GPI $(87 \%)$ or STN. Data were taken from clinical VFSS reports at baseline and post-implantation. A repeated measure ANCOVA was completed, with a dependent variable of highest PAS (per exam), a within-subjects factor of occasion (pre- vs post-surgical), betweensubjects factor laterality (left/right), and a covariate of DBS site (GPi vs STN). A post hoc 2-way Chi square test was used to assess whether proportions of subjects with increased, decreased, or unchanged PAS scores differed among hemisphere group.

Result(s): There were no significant main effects of occasion or laterality on PAS, as well as no significant interaction effects between these factors. The covariate, DBS site, did not have a significant impact. In both the left- and right-hemisphere groups, the mode PAS was 1 , before and after surgery. In the left-hemisphere group, 20 showed higher (worsened) PAS post-surgically, while 15 showed unchanged PAS and 20 showed lower (improved) PAS. In the righthemisphere group, 12 showed higher PAS post-surgically, while 10 showed unchanged PAS and 8 showed lower PAS. Differences between hemisphere groups were not significant.

Conclusions (Including Clinical Relevance): The current investigation did not reveal significant differences in PAS between left or right unilateral DBS, for either STN or GPi target. This indicates that regardless of laterality, unilateral DBS therapy may not impact swallowing safety. Study limitations include retrospective design, lack of stimulation settings data, and using only the PAS as the outcome measure. Future prospective studies should include a wider range of swallow-related outcome measures.

Relevant Financial Relationships: May Smith-Sherry: Has affiliations to disclose;University of Florida:Salary/ Stipend:Employment;UF Health:Salary/Stipend:Employment I Nicole Herndon: Has affiliations to disclose;UF Health:Salary/Stipend:Employment I Karen Hegland: Has affiliations to disclose;University of Florida:Salary/Stipend:Employment;NIH:Grant:Other Activities.

Relevant Non-financial Relationships: May Smith-Sherry: Has a Non-Financial Disclosure Affliation;DRS:Professional:Membership;ASHA:Professional:Membership I Nicole Herndon: Has a NonFinancial Disclosure Affliation;ASHA:Professional:Membership । Karen Hegland: Has a Non-Financial Disclosure Affliation;DRS:Professional:

Membership;ASHA:Professional:Membership.

\section{Effects of Two Head/Neck Strengthening Exercise Regimens on the Neuromuscular Control of Functional Swallowing in Healthy Older Adults}

\author{
Samantha Mitchell ${ }^{2}$, Robert B. Fujiki ${ }^{2}$, Abby Oliver $^{2}$, Bruce \\ $\overline{\text { Craig }}^{1}$, Georgia Malandraki ${ }^{2}$
}

Purpose: The head-lift exercise (HLE) (Shaker) and the newer recline exercise (RE) were recently shown to elicit similar biomechanical gains in older adults. However, whether and how the underlying neuromuscular control of functional swallowing changes after these regimens is not known and could provide insights on the physiological mechanisms they target. Thus, we examined and compared the effects of the HLE and the RE on this control in healthy older adults.

Method(s): A randomized clinical trial with 2 arms-a 6-week HLE or RE regimen - was conducted. Data were collected on 18 older 
adults (age range 60-82; HLE $n=9 ; \operatorname{RE} n=9$ ) pre-, post-treatment, and at 6-weeks follow-up, and included a VFSS and a surface electromyography (sEMG) study. Results of the VFSS showing hyolaryngeal excursion gains post both regimens have been published (Fujiki et al. 2019). This study presents the sEMG data. Surface EMG activity was collected from submental muscles during swallows of self-administered standardized volumes of liquids and solids. We evaluated neuromuscular control through the following outcome measures: normalized mean amplitude, burst duration, time to peak amplitude, and zero-lag cross correlations between the left and right sEMG signals.

Result(s): None of the outcome measures significantly changed across time for either group, and there were no significant differences between groups. Descriptively, amplitude decreased for more viscous boluses (cookie) post-exercise for both groups, but this decrease did not reach significance. In general, amplitude and time to peak amplitude were higher for more viscous boluses compared to thin liquid boluses $(p<0.0001)$.

Conclusions (Including Clinical Relevance): The neuromuscular control of functional swallowing did not significantly change post these regimens. When paired with the previously documented gains in hyolaryngeal excursion in this sample (Fujiki et al. 2019), we believe these results indicate that these regimens enabled the functional swallow mechanism to improve without the muscles needing to produce more energy/effort. This energy conservation offers physiological support for the use of these exercises as rehabilitative or preventative approaches. Interestingly timing did not change either, but that may be because these regimens do not target the speed of the swallow.

Relevant Financial Relationships: Samantha Mitchell: Has affiliations to disclose;Purdue University:Salary/Stipend:Employment I Robert Fujiki: Has affiliations to disclose;Purdue University:Salary/ Stipend:Employment;NIH:Salary/Stipend:Other Activities I Abby Oliver: Has affiliations to disclose;Purdue University:Salary/ Stipend:Employment;Purdue University Honors College:Grant:Other Activities I Bruce Craig: Has affiliations to disclose;Purdue University:Salary/Stipend:Employment | Georgia Malandraki: Has affiliations to disclose;Purdue University:Salary/ Stipend:Employment.

Relevant Non-financial Relationships: Samantha Mitchell: Has a Non-Financial Disclosure Affliation;NSSLHA:Professional:Membership;DRS:Professional:Membership | Robert Fujiki: Has a NonFinancial Disclosure Affliation;ASHA:Professional:Membership । Abby Oliver: Has a Non-Financial Disclosure Affliation;ASHA:Professional:Membership | Bruce Craig: Nothing to Disclose I Georgia Malandraki: Has a Non-Financial Disclosure Affliation;DRS:Professional:Board membership;ASHA:Professional:Membership;ASHA Journals:Professional:Volunteer membership on advisory committee or review panels;VA Merit Grants Panel:Professional:Volunteer membership on advisory committee or review panels.

\section{The Role of White Matter in the Neural Control of Swallowing: A Systematic Review}

\footnotetext{
Rachel Hahn Arkenberg ${ }^{1}$, Ann Alvar ${ }^{1}$, Bethany McGowan ${ }^{1}$, Hu Cheng $^{2}$, Georgia Malandraki ${ }^{3}$

Purpose: Knowledge of neural control of swallowing is primarily based on research on gray matter contributions; however, white matter is also essential, because it connects brain areas and maintains plasticity. The purpose of this study was to identify, evaluate, and summarize the populations, methods, and results of published research articles describing the role of white matter in the neural control of swallowing.
}

Method(s): We completed a systematic review with a multi-engine search conducted in accordance with PRISMA-P 2015 standards. Two authors screened all identified articles and completed blind full-text review and quality assessment with a modified NIH quality assessment. Data were extracted on populations, imaging methods/analyses, swallowing methods/analyses, and implicated white matter regions. Qualitative synthesis of evidence was completed, including identification of current gaps and opportunities for future research.

Result(s): The multi-engine search yielded 105 non-duplicate articles. Twenty two of these met inclusion criteria for full-text review, and 20 were rated as good $(5 / 22)$ or fair $(15 / 22)$ quality. Stroke was the most represented diagnosis $(n=20)$ in this literature. Only one study focused on pediatrics (cerebral palsy). All studies were observational, and half used retrospective cohort design. The majority of studies (13/22) used lesion-based methods to quantify white matter damage, with $7 / 22$ describing intrinsic characteristics of white matter using gold standard methods, like fractional anisotropy analysis. Fifteen studies used VFSS or FEES for swallowing evaluations. Despite some limitations, white matter areas commonly reported as implicated in swallowing control included the pyramidal tract, internal capsule, corona radiata, superior longitudinal fasciculus, external capsule, and corpus callosum. Noteworthy themes that emerged included: severity of white matter damage can be related to dysphagia severity; bilateral white matter lesions appear particularly disruptive to swallowing; and white matter adaptation/recovery can facilitate dysphagia recovery post-stroke.

Conclusions (Including Clinical Relevance): Despite traditionally understudied, there is sufficient evidence to conclude that white matter is critical in the neural control of swallowing. Further research is warranted on white matter's role in both dysphagia recovery and healthy swallowing to inform clinical practice in this area.

Relevant Financial Relationships: Rachel Hahn Arkenberg: Has affiliations to disclose;Purdue University:Salary/Stipend:Employment I Ann Alvar: Has affiliations to disclose;Purdue University:Salary/ Stipend:Employment I Bethany McGowan: Has affiliations to disclose;Purdue University:Salary/Stipend:Employment I Hu Cheng: Has affiliations to disclose;Indiana University:Salary/Stipend:Employment | Georgia Malandraki: Has affiliations to disclose;Purdue University:Salary/Stipend:Employment;NIH:Grant:Other Activities. Relevant Non-financial Relationships: Rachel Hahn Arkenberg: Has a Non-Financial Disclosure Affliation;American Speech Language Hearing Association:Professional:Membership;Dysphagia Research Society:Professional:Membership I Ann Alvar: Nothing to Disclose | Bethany McGowan: Nothing to Disclose | Hu Cheng: Nothing to Disclose I Georgia Malandraki: Has a Non-Financial Disclosure Affliation;American Speech Language Hearing Association:Professional:Membership;Dysphagia Research Society:Professional:Board membership;VA Merit Grants Panel:Professional:Volunteer membership on advisory committee or review panels.

\section{Capsaicin-Sensitive Nerves are Involved in the Initiation of Swallowing Evoked by Carbonated Water in Anesthetized Rats}

\section{Takanori Tsujimura $^{1}$, Midori Yoshihara ${ }^{1}$, Kouta $\operatorname{Nagoya}^{1}$, Jin Magara $^{1}$, Makoto Inoue ${ }^{1}$}

Purpose: Capsaicin powerfully evokes the swallowing reflex and is a known therapeutic agent for improving dysphagia and preventing aspiration pneumonia. However, the role of capsaicin-sensitive nerves in the initiation of swallowing evoked by various natural stimuli remains unclear. The aim of this study was to investigate the role of 
capsaicin-sensitive nerves in the initiation of swallowing evoked by mechanical and chemical stimulation.

Method(s): Experiments were performed on 108 Sprague-Dawley male rats anesthetized urethane $(1.3 \mathrm{~g} / \mathrm{kg}$, ip). For electromyographic (EMG) recordings, bipolar enamel-coated copper wire electrodes were inserted into the left suprahyoid $(\mathrm{SH})$ and thyrohyoid $(\mathrm{TH})$ muscles. Swallowing was identified by EMG bursts in the SH and TH muscles and laryngeal elevation by visual inspection. We blocked laryngeal capsaicin-sensitive nerves following the co-application of membrane-impermeant lidocaine derivative QX-314 and capsaicin (QX/Cap), and investigated the effects on swallowing evoked by mechanical and chemical stimulation in anesthetized rats. Swallows were evoked by capsaicin, carbonated water (CW), distilled water (DW), and punctate mechanical stimulation using von Frey filaments applied topically to the larynx. We also investigate the involvement of transient receptor potential (TRP) channels and acid-sensing ion channels (ASICs) in the initiation of CW-evoked swallows and the effect of bilateral transection of superior or recurrent laryngeal nerves on $\mathrm{CW}$-evoked swallows.

Result(s): The initiation of swallowing by capsaicin was strongly suppressed at $5 \mathrm{~min}$ following QX/Cap treatment and facilitated in a time-dependent manner. CW-evoked swallows at $5 \mathrm{~min}$ following QX/Cap treatment were significantly diminished compared with before and $30 \mathrm{~min}$ after treatment. In contrast, DW-evoked and mechanically evoked swallows were unchanged by QX/Cap treatment. Furthermore, CW-evoked swallows were virtually abolished by transection of the superior laryngeal nerves and significantly decreased by the topical application of non-selective ASIC inhibitor diminazene or ASIC3 inhibitor APET $\times 2$, but they were not affected by the non-selective TRP channel inhibitor ruthenium red or the ASIC1 inhibitor mambalgin-1.

Conclusions (Including Clinical Relevance): Capsaicin-sensitive nerves play an important role in the initiation of $\mathrm{CW}$-evoked swallows.

Relevant Financial Relationships: Takanori Tsujimura: Nothing to Disclose I Midori Yoshihara: Nothing to Disclose I Kouta Nagoya: Nothing to Disclose | Jin Magara: Nothing to Disclose | Makoto Inoue: Nothing to Disclose.

Relevant Non-financial Relationships: Takanori Tsujimura: Nothing to Disclose I Midori Yoshihara: Nothing to Disclose I Kouta Nagoya: Nothing to Disclose | Jin Magara: Nothing to Disclose | Makoto Inoue: Nothing to Disclose.

\section{Predictors of Dysphagia-Related Quality of Life Among Stroke Survivors}

\section{Aaliyeh Ayub $^{1}$, Hiba Najeeb ${ }^{3}$, Ashwini M. Namasivayam- MacDonald $^{3}$, Samantha Shune ${ }^{2}$}

Purpose: Dysphagia is a debilitating condition, negatively impacting survival and quality of life. As curative treatments continue to delay mortality, there is growing importance on defining treatment effectiveness in terms of life quality. While dysphagia symptoms contribute to decreased quality of life, perceived quality of life may be influenced by additional variables, including those related to the overall health of the individual. The goal of this study was to determine the independent predictors of dysphagia-related quality of life. Method(s): We collected data from 31 stroke survivors experiencing dysphagia (mean age $61.7 \pm 13.5 ; 23$ males). In addition to basic demographic information, survivors were asked about the impact of dysphagia and stroke (Swallowing-Related Quality of Life [SWALQOL], IDDSI Functional Diet Scale [IDDSI-FDS], Stroke Impact Scale [SIS]). Factors known to influence overall patient health were also collected from their caregivers, such as mental health and burden status. Variables that showed significant bivariate relationships $(p<0.20)$ with SWAL-QOL were included in a multivariate regression analysis to determine which patient and caregiver factors are associated with swallowing-related quality of life.

Result(s): Multiple survivor (age, work status, dysphagia treatment, number of medical conditions, functional health, mental health, diet restrictiveness) and caregiver variables (age, work status, burden, mental health) were significantly correlated with SWAL-QOL scores. The regression analysis revealed that survivor comorbid medical conditions $(p<0.001)$, mental health $(p=0.006)$, and IDDSI-FDS $(p=0.001)$, and caregiver work status $(p=0.001)$ were significant predictors of SWAL-QOL scores, when controlling for the other factors $\left(R^{2}=0.75 ; F(4,25)=19.13, p<0.001\right)$.

Conclusions (Including Clinical Relevance): The factors contributing to dysphagia-related quality of life are multifactorial, spanning dysphagia's consequences, and more global health. Notably, both patient- and caregiver-specific variables emerged in the subsequent model. Results support the interrelatedness of overall patient health, patient mental health, and family support, particularly for individuals coping with dysphagia-related dietary restrictions. These data highlight the need for speech-language pathologists to consider patient disability and needs more holistically in order to maximize perceived treatment outcomes.

Relevant Financial Relationships: Aaliyeh Ayub: Nothing to Disclose I Hiba Najeeb: Nothing to Disclose I Ashwini NamasivayamMacDonald: Has affiliations to disclose;McMaster University:Salary/ Stipend:Employment;American Speech-Language Hearing Association:Grant:Other Activities;Speech-language \& Audiology Canada:Grant:Other Activities I Samantha Shune: Has affiliations to disclose;American Speech-Language Hearing Association:Grant:Other Activities;University of Oregon:Salary/ Stipend:Employment.

Relevant Non-financial Relationships: Aaliyeh Ayub: Nothing to Disclose I Hiba Najeeb: Nothing to Disclose I Ashwini NamasivayamMacDonald: Has a Non-Financial Disclosure Affliation;DRS:Professional:Membership I Samantha Shune: Has a Non-Financial Disclosure Affliation;DRS:Professional:Membership.

\section{Feasibility of Device-driven Treatment Approaches for Airway Protective Deficits Via Telehealth}

\section{Jordanna Sevitz ${ }^{1}$, James C. Borders ${ }^{1}$, Avery Dakin ${ }^{1}$, Michelle S. Troche}

Purpose: Airway protective treatments frequently utilize devices; however, the feasibility of initiating these treatments via telehealth is unknown. Therefore, this study aimed to determine the feasibility of using two device-driven treatments, expiratory muscle strength training (EMST) and voluntary cough skill training (VCST) using a peak flow meter (PFM) via telehealth. We sought to identify whether patients could (1) take measures of maximum performance, (2) set treatment targets, and (3) complete exercises via teletherapy. We also explored whether factors such as disease duration, cognitive status, presence of a care-partner, and internet speed impacted participation. Method(s): 10 patients ( 2 females; age 60-82; disease duration 1-12 years) with neurodegenerative diagnoses completed 2 weeks of training. Assessments were completed via telehealth before and after treatment. Maximum expiratory pressure (MEP) was taken using the EMST device and peak expiratory flow rate (PEFR) was taken using the PFM each week and was used to set training targets. Outcomes included duration of time to obtain MEP and PEFR and complete EMST and VCST. We defined clinical feasibility as the ability to obtain measures and complete training within a 30-min session. 
Result(s): All patients successfully obtained MEP and PEFR. Mean duration to obtain MEP significantly decreased after 2 weeks from 11.95 (SD 9.03) to $5.01 \mathrm{~min}$ (SD 2.89) $(p=0.013)$. Mean duration to obtain PEFR non-significantly decreased from 4.67 (SD 2.06) to $3.30 \mathrm{~min}$ (SD 1.82, $p=0.150$ ). All patients were able to set training targets, either independently $(n=6)$ or with the assistance of a care partner $(n=4)$. Both EMST $(M=8.15 \mathrm{~min})$ and PEFR $(M=12.13 \mathrm{~min})$ practice were completed within a single session. Participant internet speeds ranged from 7.2 to 231.69 (download) and 5.27-56.63 (upload) and MMSE scores ranged from 23 to 30.

Conclusions (Including Clinical Relevance): The present study supports the feasibility of using device-driven treatment approaches via telehealth across a range of patient factors. These findings have significant implications for improving access to care for individuals with airway protective dysfunction and geographic, physical, and financial constraints. Future studies should investigate the impact of device-driven airway protective treatment paradigms via telehealth on swallowing-specific outcomes.

Relevant Financial Relationships: Jordanna Sevitz: Nothing to Disclose I James Borders: Nothing to Disclose I Avery Dakin: Nothing to Disclose I Michelle Troche: Has affiliations to disclose;Dysphagia Research Society:Honoraria:Other Activities;MedBridge Inc:Royalty:Other Activities;Teachers College, Columbia University:Salary/ Stipend:Employment;Michael J Fox Foundation:Grant:Independent contractor (Including contracted research);National Ataxia Foundation:Grant:Independent contractor (Including contracted research).

Relevant Non-financial Relationships: Jordanna Sevitz: Has a NonFinancial Disclosure Affliation;DRS Member:Professional:Membership | James Borders: Has a Non-Financial Disclosure Affliation;DRS Member:Personal:Membership I Avery Dakin: Has a Non-Financial Disclosure Affliation;DRS Member:Personal:Membership | Michelle Troche: Nothing to Disclose.

\section{Establishing Normative Values for Signs of Aspiration in Non-Dysphagic Infants}

Courtney L. Wagner ${ }^{4}$, Morgan E. Zoeller ${ }^{4}$, Christy A. Becker ${ }^{4}$, Sara E. Ramel ${ }^{1,2}$, Michael Beckstrand ${ }^{3}$, Abbey Hammell ${ }^{3}$, Katlyn E. McGrattan ${ }^{4,2}$

Purpose: Speech-language pathologists use the clinical swallow evaluation to evaluate infants for signs of swallowing impairments, such as coughing during feeds that warrant instrumental assessment. While it is accepted that occasional coughing during meals is a normal variant of function among healthy normal adults, little is known about how frequently this occurs in infants. The aim of this investigation is to fill this void by establishing objective normal correlates of feeding performance among healthy, non-dysphagic infants.

Method(s): Healthy full-term infants $<1$ week old were prospectively enrolled in the investigation. Infants were excluded if they had past or present feeding deficits, were born preterm, were exposed to prenatal drug exposure, or had an underlying medical condition known to influence feeding performance including a genetic disorder or syndrome, brain damage or abnormality, or heart defect. Consented infants underwent caregiver home monitoring of feeding performance $48 \mathrm{~h}$ a week for the first month of life. Monitoring included recording of feeding modality, feeding start and end time, and the number of coughing episodes per feed. Recorded data were uploaded weekly by caregivers using a secured web link, where caregivers would also fill out the Infant and Child Feeding Questionnaire and systemic health questions shown to be correlated with feeding difficulties. Feeding characteristics were analyzed using descriptive statistics, with changes in coughing throughout the first month of life analyzed with a paired $t$ test.
Result(s): Nineteen infants (53\% female) were enrolled in the investigation at the time of abstract submission. The majority of infants $(68 \%)$ coughed at least once each monitoring period, with the number of feeding related coughing episodes ranging from 0 to 35 times per $48 \mathrm{~h}$ data collection period. This equated to infant's coughing during an average of $15 \%$ of their feeds. No significant changes in the proportion of feeds with coughs were observed throughout the first month of life $(p=0.13)$.

Conclusions (Including Clinical Relevance): Some coughing during feeds is likely a normal variant of development among healthy term infants. Establishing normative values pertaining to feeding correlates, including signs of aspiration, are critical in guiding instrumental assessments and treatments.

Relevant Financial Relationships: Courtney Wagner: Nothing to Disclose I Morgan Zoeller: Nothing to Disclose I Christy Becker: Nothing to Disclose | Sara Ramel: Has affiliations to disclose;University of Minnesota:Salary/Stipend:Employment;Masonic Children's Hospital:Salary/Stipend:Employment;NIH, NICHD, 1R41HD104305-01:Grant:Other Activities I Michael Beckstrand: Has affiliations to disclose;University of Minnesota:Salary/Stipend:Employment I Abbey Hammell: Has affiliations to disclose;University of Minnesota:Salary/Stipend:Employment I Katlyn McGrattan: Has affiliations to disclose;University of Minnesota:Salary/Stipend:Employment;Masonic Children's Hospital:Salary/ Stipend:Employment;Dr. Brown's Medical:Speaking fee:Teaching and speaking;Biogen, Avexis:Consulting fee:Consulting;Biogen, USSPN-19-11520:Grant:Other Activities;NIH, NICHD, 1R41HD104305-01:Grant:Other Activities;nuBorn Medical:Ownership interest(e.g., stocks, stock options or other ownership interest excluding diversified mutual funds):Ownership;Science Stand:Ownership interest(e.g., stocks, stock options or other ownership interest excluding diversified mutual funds):Ownership.

Relevant Non-financial Relationships: Courtney Wagner: Nothing to Disclose I Morgan Zoeller: Nothing to Disclose I Christy Becker: Nothing to Disclose I Sara Ramel: Nothing to Disclose I Michael Beckstrand: Nothing to Disclose I Abbey Hammell: Nothing to Disclose | Katlyn McGrattan: Has a Non-Financial Disclosure Affliation;DRS Membership Committee:Professional:Volunteer membership on advisory committee or review panels;ASHA Pediatric Normal and Disordered Feeding \& Swallowing Topic Committee:Professional:Membership.

\section{Swallowing and Communication Outcomes Following Primary Transoral Robotic Surgery}

Emma Charters ${ }^{1}$, Raymond $\mathrm{Wu}^{2}$, Chris Milross ${ }^{2}$, Hans Bogaardt $^{3,6,7}$, Amy Freeman-Sanderson ${ }^{4}$, Kirrie Ballard ${ }^{3}$, Sarah Davies $^{5}$, Justine Oates ${ }^{5}$, Jonathan Clark ${ }^{5,6,7}$

Purpose: Heterogeneity within studies examining transoral robotic surgery (TORS) for oropharyngeal cancer (OPC) has made it challenging to make clear conclusions on functional outcomes. Infrequent use of instrumental swallow examinations compounds uncertainty surrounding the proposed functional advantage to TORS. The aim of this study is to perform a preliminary analysis investigating whether this policy is associated with acceptable swallowing and communication impairment by comparing patient-reported and instrumental swallow outcome measures at 12-month post-treatment in patients treated with TORS \pm adjuvant therapy with matched patients treated with radiotherapy \pm concurrent chemotherapy as a control.

Method(s): A prospective cohort of 52 patients underwent speech and swallowing assessment at 12 months following treatment for OPC. Patients were assessed using Fibreoptic Endoscopic Evaluation of Swallowing (FEES), clinician and patient reported outcomes. 
Subjects were matched according to tumor site, T category, and age. Speech and swallowing outcomes were compared for those receiving TORS vs chemo-radiation.

Result(s): When adjuvant radiotherapy to the primary site could be avoided, TORS demonstrated an advantage for feeding tube duration, secretion severity, penetration/aspiration, MDADI, airway protection, and speech handicap index (SHI). Improved results in relation to feeding tube duration, secretion severity, airway protection, MDADI score, and FOIS were lost in patients where adjuvant radiotherapy to the primary site was required.

Conclusions (Including Clinical Relevance): This explorative study suggests that a treatment philosophy of selecting patients for TORS where adjuvant therapy can be omitted or confined to the neck appears to result in favourable functional (speech and swallowing) outcomes and warrants further evaluation.

Relevant Financial Relationships: emma charters: Nothing to Disclose I Raymond Wu: Nothing to Disclose I Chris Milross: Nothing to Disclose I Hans Bogaardt: Nothing to Disclose I Amy Freeman-Sanderson: Nothing to Disclose I Kirrie Ballard: Nothing to Disclose I Sarah Davies: Nothing to Disclose I Justine Oates: Nothing to Disclose I Jonathan Clark: Nothing to Disclose.

Relevant Non-financial Relationships: emma charters: Nothing to Disclose I Raymond Wu: Nothing to Disclose I Chris Milross: Nothing to Disclose I Hans Bogaardt: Nothing to Disclose I Amy FreemanSanderson: Nothing to Disclose I Kirrie Ballard: Nothing to Disclose I Sarah Davies: Nothing to Disclose I Justine Oates: Nothing to Disclose I Jonathan Clark: Nothing to Disclose.

\section{Disparities Research in Oropharyngeal Dysphagia: A Scoping Review}

\author{
Anaïs Rameau ${ }^{2,4}$, Noah Feit ${ }^{1}$, Valentina Mocchetti ${ }^{2,4}$, Moon \\ Usman $^{1}$, Zhaorui Wang ${ }^{1}$, Michelle Demetres ${ }^{3}$, Katerina \\ Andreadis ${ }^{2,4}$
}

Purpose: This review intends to highlight existing primary healthcare disparities research in oropharyngeal dysphagia with the objective of elucidating knowledge gaps and guiding future prospective intervention-based research.

Method(s): A scoping review of three bibliographic databases, including PubMed, Ovid Embase, and the Cochrane Library, was executed in accordance with PRISMA extension for scoping reviews (PRISMA-ScR). Primary research evaluating disparities in the burden of disease, treatment strategies, survival, and other metrics of inequity were sought. Inequities considered were limited to race/ethnicity, sex/gender, insurance status, education level, income, geography, and LGBTQ identity. Publication of any design and date, performed in the US, and focusing on the adult population exclusively were included. Result(s): Of the 1049 PubMed, 701 Ovid Embase, and 91 Cochrane Library results, 7 articles represented primary disparities research in oropharyngeal dysphagia. In all 7 results, swallowing dysfunction was secondary to stroke. In 5 of the 7 articles, percutaneous endoscopic gastrostomy (PEG) tube placement was the primary outcome of interest. The most frequently examined source of inequity was race/ ethnicity ( 7 of 7 [100.0\%] studies), followed by sex/gender (5 of 7 [71.4\%] studies), education status (3 of 7 [42.9\%] studies), income (2 of 7 [28.6\%] studies), geography (2 of 7 [28.6\%] studies), and education status ( 1 of 7 [14.3\%] studies). There were an absence of studies assessing swallowing-related disparities in the context of LGBTQ identity. No result entirely rejected the presence of inequity in the domain it intended to investigate. Senior authors were either neurologists (5 of 7 [71.4\%] studies) or physiatrists ( 2 of 7 [28.6\%] studies) with no representation by otolaryngologists or speech pathologists.
Conclusions (Including Clinical Relevance): This scoping review identifies the limited research on healthcare disparities in oropharyngeal dysphagia in the US adult population. Although multiple sources of disparities are revealed, further research to address inequities related to identification, evaluation, and treatment of oropharyngeal dysphagia across various health disciplines and settings is needed.

Relevant Financial Relationships: Anaïs Rameau: Nothing to Disclose I Noah Feit: Nothing to Disclose I Valentina Mocchetti: Nothing to Disclose I Moon Usman: Nothing to Disclose I Zhaorui Wang: Nothing to Disclose I Michelle Demetres: Nothing to Disclose I Katerina Andreadis: Nothing to Disclose.

Relevant Non-financial Relationships: Anaïs Rameau: Has a NonFinancial Disclosure Affliation;DRS:Professional:Membership I Noah Feit: Nothing to Disclose I Valentina Mocchetti: Has a Non-Financial Disclosure Affliation;DRS:Professional:Membership | Moon Usman: Nothing to Disclose I Zhaorui Wang: Nothing to Disclose I Michelle Demetres: Nothing to Disclose I Katerina Andreadis: Has a NonFinancial Disclosure Affliation;DRS:Professional:Membership.

\section{Laryngeal Deficits Following Cervical Spinal Cord Injury and Evidence for a Novel Therapy}

\section{Teresa Pitts ${ }^{1}$, Clint Greene ${ }^{1}$, Kimberly Iceman ${ }^{1}$, Dena R. Howland $^{1,2}$}

Purpose: Laryngeal dysfunction is a common symptom following cervical spinal cord injury (cSCI). Although the mechanism is understood, cSCI can result in laryngeal deficits and dysphagia. Our previous terminal electrophysiology experiments with acute hemisections at the 3rd cervical spinal level (C3) showed immediate alterations in laryngeal activity. These alterations persisted for $4 \mathrm{~h}$ (testing duration). The serotonin $\left(5-\mathrm{HT}_{1 \mathrm{~A}}\right)$ receptor agonist $8-\mathrm{OH}-$ DPAT was delivered via the vertebral artery at low $(3 \mu \mathrm{g} / \mathrm{kg})$ and $/$ or high $(30 \mu \mathrm{g} / \mathrm{kg})$ doses. This agonist altered behavior-specific muscle recruitment patterns. In particular, the high dose restored laryngeal activity across breathing and swallow, which could be reversed with the competitive antagonist WAY100635 $(0.5 \mathrm{mg} / \mathrm{kg})$.

Method(s): In the current study, we tested if alterations seen acutely in laryngeal activity persist chronically, and if the 8-OH-DPAT clinical-correlate buspirone (Buspar) would produce similar therapeutic results.

Result(s): Adult female cats underwent $\mathrm{C} 3$ hemisections and recovered for 4 or 8 weeks. Laryngeal endoscopy was performed weekly under isofluorane (3\%) during normocapnia and hypercapnia $(5 \%$ $\mathrm{CO}_{2}$ ). Asynchronous vocal fold movement was seen in all animals, defined by unilateral movement delays, and the extent of opening and closing of the glottis became variable. In a subset of animals, oral buspirone $(5 \mathrm{mg}$ ) was given with food. One hour later, repeat endoscopic evaluation revealed full resolution of laryngeal function with synchronized movements during normocapnia and hypercapnia. Most importantly, with termination of buspirone dosing, laryngeal dysfunction returned.

Conclusions (Including Clinical Relevance): This work shows that the anesthetized preparation effectively identifies upper airway dysfunction early and chronically after C3 SCI, and that it can be used as a pre-clinical model for novel therapeutic interventions. Results also indicate that buspirone is a high-priority target for additional investigation as a therapeutic intervention after cSCI. This work was supported by NIH grants HL 111215, HL 103415, and OT20D001983, the Craig H. Neilsen Foundation Pilot Research Grant 546714, Veterans Affairs Rehabilitation, Research and Development RCSB9249S (DRH), Kentucky Spinal Cord and Head Injury 
Research Trust, Rebecca F. Hammond Endowment (DRH), and the Commonwealth of Kentucky Challenge for Excellence.

Relevant Financial Relationships: Teresa Pitts: Nothing to Disclose | Clint Greene: Nothing to Disclose I Kimberly Iceman: Nothing to Disclose I Dena Howland: Nothing to Disclose.

Relevant Non-financial Relationships: Teresa Pitts: Nothing to Disclose I Clint Greene: Nothing to Disclose I Kimberly Iceman: Nothing to Disclose I Dena Howland: Nothing to Disclose.

\section{Respiratory-Related Sensory Decline and Swallow Safety in People with PD: Preliminary Longitudinal Data}

\begin{abstract}
Michela Mir ${ }^{1}$, Michelle Slepian², Karen Hegland ${ }^{2,1}$
Purpose: Control of airway protection depends on intact sensory input from the respiratory tract to mediate ventilation and coordinate swallow and cough for pulmonary health. People with Parkinson's disease (PD) exhibit a blunted perception of respiratory sensations related to swallow and cough dysfunction. Yet, the onset of this sensory decline compared to swallowing impairment is unknown. We aimed to determine differences in sensory responses from airway stimuli and measures of swallow safety in people with PD across 10-18 months. We hypothesized that in early disease, swallow safety would remain the same despite a decline in sensory responses.

Method(s): Participants (32 male; 6 female) completed the following tasks at baseline (Y1) and 10-18 months later (Y2). Upon inspiring through resistive loads (5 levels +1 control), we recorded participants' ratings of perceived breathing difficulty $(6=$ no; $20=\max )$. During induced cough challenges (4 levels aerosolized capsaicin + control), we recorded their perceived urge-to-cough (UtC; $0=$ no; $10=\max$ ) and the lowest level to which they coughed twice (C2). We collected penetration-aspiration scale (PAS) scores, cough responses, and $\mathrm{UtC}$ ratings after participants swallowed thin liquid barium $(10 \mathrm{ml} \times 3 ; 90 \mathrm{ml} \times 1)$ during videofluoroscopic swallow studies. Related samples of $t$ test were used to determine differences and nonparametric tests for ordinal variables.
\end{abstract}

Result(s): Sensory response measures indicated that ratings of breathing difficulty remained constant between $\mathrm{Y} 1$ and $\mathrm{Y} 2$, UtC sensitivity slope decreased significantly $(p<0.011, d=0.44)$, and a non-significant increase in capsaicin was required to reach $\mathrm{C} 2$ threshold. Although there were no significant differences between PAS scores in $\mathrm{Y} 1$ and $\mathrm{Y} 2$, there were significantly more unsafe swallows with $90 \mathrm{ml}$ trial compared to $10 \mathrm{ml}$ in $\mathrm{Y} 1$ and $\mathrm{Y} 2$ $(p<0.001 ; p=0.019)$. Median Hoehn and Yahr score for this cohort were 2.0 and mean disease duration was 6.92 years.

Conclusions (Including Clinical Relevance): Between Y1 and Y2, cough sensitivity to aerosolized capsaicin significantly decreased, while other measures of respiratory sensations and PAS scores remained similar. Although preliminary, these results partially support our hypothesis that the onset of airway sensory decline may occur prior to swallow safety decline in early PD and identification of this deficit may prompt proactive sensorimotor treatments for swallow and cough functions.

Relevant Financial Relationships: Michela Mir: Has affiliations to disclose;University of Florida T32 Neuromuscular Plasticity Predoctoral fellowship:Salary/Stipend:Other Activities I Michelle Slepian: Has affiliations to disclose;University of Florida:Salary/ Stipend:Employment I Karen Hegland: Has affiliations to disclose;National Institutes of Health:Grant:Other Activities;University of Florida:Salary/Stipend:Employment.
Relevant Non-financial Relationships: Michela Mir: Has a NonFinancial Disclosure Affliation;Dysphagia Research Society:Professional:Membership;American Speech-Language Hearing Association:Professional:Membership;National Foundation of Swallow Disorders:Personal:Membership I Michelle Slepian: Has a NonFinancial Disclosure Affliation;American Speech Language Hearing Association:Professional:Membership I Karen Hegland: Has a NonFinancial Disclosure Affliation;Dysphagia Research Society:Professional:Membership;American Speech-Language Hearing Association:Professional:Membership.

\section{Age Influences the Impact of Stroke on Bihemispheric Motor Cortex Connectivity to the Jaw and Tongue Muscles}

\section{Miranda J. Cullins ${ }^{1}$, Nadine Connor ${ }^{2}$}

Purpose: Dysphagia commonly occurs after stroke, yet the mechanisms of corticobulbar plasticity after stroke are not well understood. Due to the bihemispheric control of swallowing, it has been suggested that plasticity of the contralesional (intact) cortex drives recovery of swallowing. Age may be an important factor in that stroke most commonly occurs in aged individuals, age is an independent predictor of dysphagia after stroke, and neuroplasticity may be reduced with age. We sought to determine the impact of unilateral stroke and age on cortical plasticity by examining the volume of the sensorimotor cortex that activates the jaw and tongue muscles in both hemispheres.

Method(s): Using the middle cerebral artery (MCA) occlusion rat stroke model, intracortical microstimulation (ICMS) was used to map regions of sensorimotor cortex that activate tongue and jaw muscles in both hemispheres. Larger cortical maps suggest greater functional connectivity for muscles important in sensorimotor control of swallowing. Young adult ( 7 months) and aged (30 months) F344xBN rats underwent either a stroke via left middle cerebral artery occlusion or a control sham surgery, followed by ICMS mapping 8 weeks later. Preliminary data reflected in this abstract included the following sample sizes: 5 Aged Stroke, 5 Aged Sham, 4 Young Stroke, and 3 Young Sham. Samples sizes will be increased to 10 per group before the conference and all data will be included.

Result(s): The volume of ipsilesional sensorimotor cortex that activates both the jaw and tongue was reduced with stroke $(p<0.001)$. The jaw-activating region of the contralesional cortex increased in the stroke group vs. sham $(p=0.041)$. Within the aged stroke group, the remaining region of ipsilesional cortex that activates the jaw and tongue was significantly smaller than in the young adult stroke group $(p=0.007)$.

Conclusions (Including Clinical Relevance): MCA stroke is associated with a significant loss of functional connectivity between the sensorimotor cortex and the muscles of the jaw and tongue, which is greater with age. This reduced cortical input is likely to represent reduced volitional control of the swallowing musculature. An increase in the jaw representation in the intact hemisphere is in agreement with studies that have suggested a role for contralesional cortical plasticity in the recovery of oral-motor function after stroke.

Relevant Financial Relationships: Miranda Cullins: Nothing to Disclose I Nadine Connor: Nothing to Disclose.

Relevant Non-financial Relationships: Miranda Cullins: Has a NonFinancial Disclosure Affliation;DRS:Professional:Membership । Nadine Connor: Has a Non-Financial Disclosure Affliation;DRS:Professional:Membership. 
Device-Facilitated Lingual Strengthening in Veterans with Head and Neck Cancer Following Oncologic Treatment: Feasibility and Swallowing-Related Outcomes

Carly M. Gurick ${ }^{1}$, Joanne Yee ${ }^{1,2}$, Loni Arrese ${ }^{2,4}$, Fauzia Osman ${ }^{2}$, Katherine Yetter $^{5}$, Kenton Woo ${ }^{6}$, Jenna Thompson ${ }^{10,11}$, Brittany N. Krekeler ${ }^{7}$, Katherine A. Hutcheson ${ }^{8,9}$, Nicole Rogus-Pulia ${ }^{1,2,3}$

Purpose: The role of device-facilitated lingual strengthening (LS) in patients with oropharyngeal dysphagia after head and neck cancer (HNC) treatment remains unclear. The purpose of this study was to assess feasibility and examine swallowing-related outcomes of LS in a cohort of patients treated for HNC.

Method(s): A retrospective analysis of Veterans enrolled in a standardized, intensive clinical dysphagia treatment program across three sites was conducted. Inclusion criteria were (1) HNC diagnosis; (2) completion of oncologic treatment; (3) dysphagia on a modified barium swallow study (MBS); and (4) participation in an 8-week device-facilitated isometric progressive LS treatment protocol. Feasibility was assessed through treatment completion rates and adherence data recorded in the device. Outcomes data were collected by practicing clinicians trained in standard protocols and recorded in a central database. Maximum lingual pressures at anterior and posterior tongue locations were recorded. Patient-reported outcome measures relating to swallow effort and quality of life were collected. Functional oral intake scale (FOIS) scores were recorded. Dynamic grade of swallowing toxicity (DIGEST) scores were derived for a subset of 22 patients with MBS images at both assessment points.

Result(s): 38 Veterans (mean age $=70$ years, mean 81.1 months survival time) were included. The LS regimen completion rate was $89.9 \%$. Adherence to exercise was $66.8 \%$ (anterior sensor) and $62.2 \%$ (posterior sensor). Following LS, patients reported significant improvements in swallowing quality of life total scores $(p<0.001)$ and seven subscale scores $(p<0.05)$. Patients reported increased appetite $(p<0.008)$ and less perceived swallowing effort $(p<0.02)$. Anterior and posterior lingual pressures increased (mean change $=$ 37.4 and $32.6 \mathrm{hPA}$, respectively) but did not reach statistical significance $(p=0.05$ and 0.08$)$. Multiple linear regression revealed baseline lingual pressure as the only significant predictor of lingual pressure change. FOIS and DIGEST scores did not change.

Conclusions (Including Clinical Relevance): These findings highlight feasibility of a LS regimen in the HNC population as indicated by program completion and adherence rates. In addition, data suggest that a LS regimen may positively impact quality of life, swallowing effort, and lingual pressure generation; however, carryover-to-swallowing function may be limited and requires further study.

Relevant Financial Relationships: Carly Gurick: Has affiliations to disclose;William S. Middleton Memorial Hospital:Salary/ Stipend:Employment | Joanne Yee: Has affiliations to disclose;Department of Veterans Affairs:Salary/Stipend:Employment I Loni Arrese: Has affiliations to disclose;Ohio State University:Salary/ Stipend:Teaching and speaking I Fauzia Osman: Nothing to Disclose I Katherine Yetter: Nothing to Disclose I Kenton Woo: Nothing to Disclose I Jenna Thompson: Nothing to Disclose I Brittany Krekeler: Nothing to Disclose I Katherine Hutcheson: Nothing to Disclose I Nicole Rogus-Pulia: Has affiliations to disclose;University of Wisconsin Madison:Salary/Stipend:Employment;University of Wisconsin Medical Foundation:Salary/Stipend:Employment;William S. Middleton VA Hospital:Salary/Stipend:Employment.

Relevant Non-financial Relationships: Carly Gurick: Nothing to Disclose | Joanne Yee: Has a Non-Financial Disclosure Affliation;Dysphagia Research Society:Professional:Membership | Loni Arrese: Has a Non-Financial Disclosure Affliation;Dysphagia Research Society:Professional:Membership | Fauzia Osman: Nothing to Disclose I Katherine Yetter: Nothing to Disclose I Kenton Woo: Nothing to Disclose I Jenna Thompson: Nothing to Disclose I Brittany Krekeler: Nothing to Disclose I Katherine Hutcheson: Nothing to Disclose I Nicole Rogus-Pulia: Has a Non-Financial Disclosure Affliation;Dysphagia Research Society:Professional:Membership;Dysphagia Research Society Board of Directors:Professional:Board membership.

\section{Respiratory-Swallow Training in Head and Neck Cancer: Impact on Essential Timing of Movements for Airway Protection and Efficiency}

Brittany N. Krekeler ${ }^{1}$, Cagla Kantarcigil ${ }^{1}$, Kate Davidson ${ }^{1}$, Kent $\overline{\text { Armeson }}^{2}$, Bonnie Martin-Harris ${ }^{1}$

Purpose: Timing of swallowing initiation during respiration (inspiration vs expiration) impacts swallow safety and efficiency. For patients with dysphagia after head and neck cancer (HNC) treatment, initiating swallowing during inspiration has been closely linked with aspiration and swallowing impairments. A clinical trial (MartinHarris et al. 2015) demonstrated that training initiation of swallowing during expiration (Respiratory-Swallow Training, RST) in a cohort of patients with chronic dysphagia after treatment for HNC leads to physiological benefits, including significant improvements in tongue base retraction (TBR), laryngeal vestibular closure (LVC), and airway protection. The current study investigates the impact of RST on timing of swallowing movements underlying these noted improvements in swallowing physiology.

Method(s): Using Adobe Premiere for frame-by-frame analysis of modified barium swallow studies (MBSS) completed before and after RST, we measured duration of: oral transit time (OTT-onset of lingual propulsion to bolus passing ramus), laryngeal elevation, (LE-first upward movement of larynx to return to rest), LVC (epiglottic-arytenoid contact to opening of vestibule), TBR (onset of retraction to release), hyoid excursion (HE-hyoid burst to return to rest), and pharyngoesophageal (PES) opening (opening to max). MBSS bolus presentations included two trials of $5 \mathrm{ml}, 15 \mathrm{ml}$, and self-selected cup sip of standard Varibar contrasts (thin, nectar, honey) presented in sequential order. Bolus trials were grouped and averaged. One sample, two-sided $t$ tests analyzed the average paired differences from pre- to post-treatment $(\alpha \leq 0.05)$.

Result(s): Analyses of MBSS from 7 participants with chronic dysphagia ( $>1$ year) after HNC treatment showed that after RST ,the durations of the following increased: LVC $(p=0.016)$, LE $(p=0.0075)$, TBR $(p=0.026)$, HE $(p=0.047)$, and PES opening $(p=0.037)$ decreased. There were no changes in duration of OTT $(p=0.51)$.

Conclusions (Including Clinical Relevance): RST resulted in increased duration of critical swallowing events that contribute to swallowing safety (i.e., laryngeal vestibular closure, elevation, and hyoid excursion) and efficiency (i.e., tongue base retraction, PES opening). These findings support previously reported clinical trial data showing improvements in physiologic swallowing components (TBR, LVC) and improved airway safety (penetration-aspiration scale scores) as a result of RST.

Relevant Financial Relationships: Brittany Krekeler: Has affiliations to disclose;Northwestern University:Salary/ Stipend:Employment I Cagla Kantarcigil: Has affiliations to disclose;Northwestern Univeristy:Salary/Stipend:Employment I Kate Davidson: Has affiliations to disclose;Northwestern University:Salary/Stipend:Employment;MBSImP:Salary/Stipend:Employment I Kent Armeson: Has affiliations to disclose;Medical University of South Carolina:Salary/Stipend:Employment | Bonnie Martin- 
Harris: Has affiliations to disclose;Northwestern University:Salary/ Stipend:Employment;MBSImP:Royalty:Ownership.

Relevant Non-financial Relationships: Brittany Krekeler: Has a Non-Financial Disclosure Affliation;DRS:Professional:Membership I Cagla Kantarcigil: Has a Non-Financial Disclosure Affliation;DRS:Professional:Membership | Kate Davidson: Has a NonFinancial Disclosure Affliation;DRS:Professional:Membership I Kent Armeson: Nothing to Disclose I Bonnie Martin-Harris: Has a NonFinancial Disclosure Affliation;DRS:Professional:Membership.

\section{Lingual Accommodation Based on Bolus Characteristics: Pressure Patterns in Oral Tongue and Tongue Base in Healthy Adults}

\section{Brittany N. Krekeler ${ }^{1,2}$, Kazuhiro Hori ${ }^{3}$, Corinne A. Jones ${ }^{4}$, Sarah Rosen $^{2}$, Suzan Abdelhalim ${ }^{2}$, Timothy Mcculloch ${ }^{2}$, Nicole Rogus-Pulia ${ }^{5,2,6}$}

Purpose: Efficient bolus transport requires precise coordination of oral musculature for adequate pressure generation to clear material from the oropharynx. Bolus characteristics (volume/consistency) have been shown to affect timing and magnitude of lingual movements. However, less is known about the relationship between oral tongue (OT) and tongue base (TB) pressure generation during swallowing and whether this is influenced by bolus type. The present investigation is the first to compare simultaneous recordings of OT and TB pressures to determine how these regions synergize to accommodate different bolus types.

Method(s): Healthy volunteers $(N=20$, mean age $=43$, range 19-72, 7 female) were recruited for simultaneous measurement of OT and TB pressures during swallowing of varying bolus types $(5 \mathrm{cc}$, $10 \mathrm{cc}, 20 \mathrm{cc}$; IDDSI $0,2,3)$ in a randomized order with three trials per bolus. OT pressures were recorded via Swallow Scan, consisting of 5 embedded sensors (OT1-5) adhered to the hard palate. TB pressures were recorded from two high-resolution manometry sensors (superior $=$ TB1; inferior $=$ TB2). RM ANOVAs $(\alpha=0.05)$ were used to detect effect of bolus type on (1) max pressure recorded at each OT and TB sensor and (2) latency (ms) between max pressure recordings at each sensor.

Result(s): As bolus volume increased, greater pressures were generated by the anterior OT region against the hard palate (OT1, $p=0.02)$. In contrast, pressures at the mid OT region (OT2, $p=0.03$ ) were increased for smaller volumes. As consistency increased, greater pressures were generated by posterior OT regions (OT3-5, $p<0.03)$. TB1 max pressures were greater both with increasing volume $(p=0.004)$ and consistency $(p=0.02)$. TB2 max pressures $(p=0.01)$ showed an interaction effect, with greater pressures observed with smaller, moderately thick boluses. Latency between max pressures at posterior OT regions (OT3-4) and TB1 were longer with larger volumes $(p \leq 0.01)$.

Conclusions (Including Clinical Relevance): These data show that max pressures in the OT and TB are differentially generated based on bolus properties in healthy individuals across the age span. These findings highlight the complex connection between lingual movements required for normal swallowing and the importance of synergy among lingual regions to accommodate variations in bolus type. Future work will investigate these how lingual movements vary based on bolus type in individuals with dysphagia.

Relevant Financial Relationships: Brittany Krekeler: Has affiliations to disclose;Northwestern Univeristy:Salary/ Stipend:Employment I Kazuhiro Hori: Has affiliations to disclose;Niigata University:Salary/Stipend:Employment I Corinne Jones: Has affiliations to disclose;UT-Austin:Salary/Stipend:Employment I Sarah Rosen: Has affiliations to disclose;UW-Madison:Salary/
Stipend:Employment I Suzan Abdelhalim: Has affiliations to disclose;UW-Madison:Salary/Stipend:Employment I Timothy Mcculloch: Has affiliations to disclose;UW-Madison:Salary/ Stipend:Employment I Nicole Rogus-Pulia: Has affiliations to disclose;UW-Madison:Salary/Stipend:Employment.

Relevant Non-financial Relationships: Brittany Krekeler: Has a Non-Financial Disclosure Affliation;DRS:Professional:Membership I Kazuhiro Hori: Nothing to Disclose I Corinne Jones: Has a NonFinancial Disclosure Affliation;DRS:Professional:Membership । Sarah Rosen: Nothing to Disclose I Suzan Abdelhalim: Nothing to Disclose I Timothy Mcculloch: Has a Non-Financial Disclosure Affliation;DRS:Professional:Membership I Nicole Rogus-Pulia: Has a Non-Financial Disclosure Affliation;DRS:Professional:Membership.

\section{The Role of Aspiration Amount on Airway Protective Response in People with Neurogenic Dysphagia}

$\underline{\text { James A. Curtis }}^{1}$, James C. Borders ${ }^{1}$, Avery Dakin ${ }^{1}$, Michelle S. Troche

Purpose: The primary aim of this study was to assess the effects of aspiration amount on ejective airway protective responses (e.g., cough, throat clear) in people with neurogenic dysphagia. Secondary aims were to assess the effects of bolus volume, disease diagnosis, and disease duration on airway protective responses after accounting for aspiration amount.

Method(s): A secondary analysis of data collected from people with neurogenic dysphagia undergoing a flexible endoscopic evaluation of swallowing (FEES) was completed. FEES included 5, 10, 15, selfadministered, and $90 \mathrm{~mL}$ thin liquid boluses. The PAS was rated for each swallow. Only swallows with PAS 6-7 (aspiration with an effort to eject) or PAS 8 (aspiration with no effort to eject) were included. Aspiration amount was defined as the amount of subglottic surface area covered by aspirate material after the swallow and was quantified using a 100-point visual analog scale. A binomial generalized mixed model was used to examine if aspiration amount influenced the likelihood of airway protective responses. Separate models were used to examine if bolus volume, disease diagnosis, and disease severity influenced the likelihood airway protective responses after accounting for aspiration amount.

Result(s): 144 swallows with a PAS 6-7 and 237 swallows with a PAS 8 were identified across 139 FEES from 85 participants. Statistical analyses revealed a significant effect of aspiration amount on the likelihood of airway protective responses, with a greater amount of aspiration associated with a higher likelihood of airway protective response indicated by PAS 6 or $7(p<0.0005 ; d=0.454)$. Additional modeling revealed that, after accounting for aspiration amount ( $p<0.0005)$, neither bolus volume $(p=0.078 ; d=0.089)$, disease duration $(p=0.293, d=0.004)$, nor disease diagnosis $(p=0.106$, $d=0.084$ ) influenced the likelihood of airway protective responses. Conclusions (Including Clinical Relevance): This study demonstrates that airway protective responses to aspiration of liquids are largely dependent on the amount of aspiration in people with neurogenic dysphagia. Furthermore, bolus volume and disease-specific factors do not appear to influence response to aspiration after accounting for aspiration amount. Future research is needed to comprehensively understand the influence of other bolus and patientspecific factors on airway protective responses in PD and other populations.

Relevant Financial Relationships: James Curtis: Has affiliations to disclose;Teachers College, Columbia University:Salary/Stipend:Employment;American Brain Foundation:Grant:Independent contractor (Including contracted research);Parkinson's Foundation:Grant:Independent contractor (Including contracted research);American 
Academy of Neurology:Grant:Independent contractor (Including contracted research) I James Borders: Nothing to Disclose I Avery Dakin: Nothing to Disclose I Michelle Troche: Has affiliations to disclose;Teachers College, Columbia University:Salary/Stipend:Employment;Medbridge Inc.:Royalty:Other Activities;Dysphagia Research Society:Honoraria:Other Activities;Michael J Fox Foundation:Grant:Independent contractor (Including contracted research);National Ataxia Foundation:Grant:Independent contractor (Including contracted research).

Relevant Non-financial Relationships: James Curtis: Has a NonFinancial Disclosure Affliation;Dysphagia Research Society:Professional:Membership;Dysphagia Research Society:Professional: Other volunteer activities I James Borders: Has a Non-Financial Disclosure Affliation;Dysphagia Research Society:Professional:Membership । Avery Dakin: Has a Non-Financial Disclosure Affliation;Dysphagia Research Society:Professional:Membership | Michelle Troche: Has a Non-Financial Disclosure Affliation;Dysphagia Research Society:Professional:Membership.

\section{D Computer Graphics Model of the Anatomy and Physiology of Babies' Sucking and Swallowing as an Educational Tool}

Flávia R. Puccini ${ }^{5}$, Roberta Martinelli ${ }^{4}$, Antônio D. Rodrigues ${ }^{1}$, Marina Gatti ${ }^{3}$, Chao L. Wen ${ }^{2}$, Giédre Berretin-Felix ${ }^{3}$

Purpose: Breastfeeding is a complex function that requires coordination between sucking, swallowing, and breathing. Although technological and scientific advances have made it possible to better understand the steps involved in that process, no scientifically validated tool has been found in the literature that shows the anatomy and physiology of babies' sucking and swallowing through video. The current study aimed to develop and validate a 3D computer graphics sequence content showing babies' sucking and swallowing functions during breastfeeding as an educational tool.

Method(s): Two speech-language pathologists and one anatomist prepared a scientific script to develop the 3D graphic material. The script was based on the literature and contained information on newborns' face, skull, oral cavity, pharynx, and esophagus anatomy and the description of mechanisms related to the physiology of babies' sucking and oral and pharyngeal phases. A conceptual map with definitions of the related subjects and topics associated with macro-themes and interrelationships was designed. Also, a matrix of content, objectives, and competencies was built. The virtual baby's sucking and swallowing production included modeling, animation, texturing, lighting, rendering, composition, and post-production process and was performed by two web designers in partnership with the Virtual Man Project. The results were analyzed using descriptive statistics.

Result(s): 3D computer graphics enabled the professionals to create interactive educational material that includes static images and dynamic sequences on the anatomy and physiology of babies' sucking and swallowing during breastfeeding. After evaluation, the content and iconographic images were adjusted to match the literature findings, thus, verifying the items' representativeness with the bibliographic findings at the end of the process.

Conclusions (Including Clinical Relevance): The study described the process of building 3D iconographic images of a virtual, term newborn's sucking and swallowing as well as validated the material, thus, verifying the reliability of the content, which represents an important resource aimed at health education both in training future professionals and the general population and contributes to health promotion.
Relevant Financial Relationships: Flávia Puccini: Nothing to Disclose I Roberta Martinelli: Nothing to Disclose I Antônio Rodrigues: Nothing to Disclose I Marina Gatti: Nothing to Disclose I Chao Wen: Nothing to Disclose I Giédre Berretin-Felix: Nothing to Disclose.

Relevant Non-financial Relationships: Flávia Puccini: Nothing to Disclose I Roberta Martinelli: Nothing to Disclose I Antônio Rodrigues: Nothing to Disclose I Marina Gatti: Nothing to Disclose I Chao Wen: Nothing to Disclose | Giédre Berretin-Felix: Nothing to Disclose.

\section{Dysphagia Profiles and Management Patterns in Patients with COVID-19}

\section{$\underline{\text { Michael Pulia }^{1}, \text { Rebecca Schwei }^{1} \text {, Nicole Rogus-Pulia }}{ }^{2,3}$}

Purpose: The prevalence of dysphagia in patients with coronavirus disease 2019 (COVID-19) is poorly characterized. The purpose of this study was to describe pre-existing and post-COVID-19 dysphagia profiles and management among patients with confirmed COVID-19.

Method(s): Data on consecutive, symptomatic patients who tested positive for COVID-19 in two Midwest emergency departments, between $3 / 15 / 20$ and $8 / 11 / 20$, was extracted from the electronic health record (EHR). All outcomes were abstracted through 30 days postindex ED visit. All variables were defined a priori with abstractions conducted by blinded research coordinators. Patients with a dysphagia history were identified using the search feature of the EHR that enables capture of any instance of this specific term. Subsequently, detailed pre/post-COVID-19 dysphagia history and management data were abstracted. Descriptive statistics are presented.

Result(s): Among a consecutive cohort of 138 patients with COVID$19,23(16.6 \%)$ had a prior documented history of dysphagia. A total of 7 patients $(5.1 \%)$ were newly diagnosed with dysphagia postCOVID-19, 3 following intubation. The median age of the entire dysphagia cohort (30 patients) was 71 years with $60 \%$ males and 13 $(43.3 \%)$ having a history of dementia. The median, worst WHO COVID-19 stage was 4 (range 1 to 8 ). Of the 23 patients with preexisting dysphagia, $17(73.9 \%)$ had a documented swallow evaluation prior to their COVID-19 diagnosis, including 15 (65.2\%) who underwent a videofluoroscopic swallowing study (VFSS) and 13 $(59.4 \%)$ who were on modified diets. Among those with a VFSS, penetration and/or aspiration were noted in $12(52.2 \%)$ cases. Across both groups, post-COVID dysphagia evaluations included bedside swallow evaluation in 12 cases $(40 \%)$ and instrumental exams in 5 cases (16.6\%: 4 VFSS, 1 FEES).

Conclusions (Including Clinical Relevance): Preexisting dysphagia was prevalent ( 1 out of 6 ) in a consecutive cohort of patients with confirmed COVID-19. New onset dysphagia post-COVID-19 was relatively uncommon as were post-COVID-19 instrumental exams. The prevalence of preexisting dysphagia among a group of primarily older adults, nearly half of whom had dementia, suggests a potential unexplored independent risk factor for disease acquisition. Future studies should explore the contribution of dysphagia to COVID-19 susceptibility and severity in older adults.

Relevant Financial Relationships: Michael Pulia: Nothing to Disclose I Rebecca Schwei: Nothing to Disclose I Nicole Rogus-Pulia: Nothing to Disclose.

Relevant Non-financial Relationships: Michael Pulia: Nothing to Disclose I Rebecca Schwei: Nothing to Disclose I Nicole Rogus-Pulia: Has a Non-Financial Disclosure Affliation;Dysphagia Research Society Member:Professional:Membership;Dysphagia Research Society:Professional:Board membership. 
Auto Segmentation of the Hyoid Bone, Bolus, and C2C4 Vertebrae Length on Videofluoroscopic Swallow

\section{Studies}

\section{Nadeem M. Shaheen ${ }^{1}$, Rodolfo Peña-Chávez ${ }^{2}$, Chris Ulmschneider $^{3}$, Ryan Burdick ${ }^{2,4}$, Joanne Yee ${ }^{5,4}$, Atsuko Kurosu ${ }^{3}$, Nicole Rogus-Pulia ${ }^{2,4,5}$, Bryan Bednarz ${ }^{1}$}

Purpose: The hyoid bone, cervical vertebrae, and the bolus are regions of interest (ROIs) identified in videofluoroscopic swallow studies (VFSS) that contain information relevant to commonly used clinical measures for diagnosing swallowing disorders. Analysis of these ROIs in VFSS is time consuming and often requires a variety of manual inputs, adjustments, or measures to extract accurate anatomical and physiologic information. This study aimed to evaluate the accuracy of an auto-segmentation algorithm of the hyoid bone, bolus, and cervical vertebrae 2 (C2) and 4 (C4) in VFSS image sequences. Method(s): Eleven VFSS image sequences of thin liquid swallows recorded at 30 frames per second were selected from a cohort of patients with post-stroke dysphagia. The first swallow was manually cropped from full-length videos resulting in 11 anatomically unique image sequences. Images were scaled from their native shape to $256 \times 256$. The hyoid, bolus, C2, and C4 were manually segmented across every frame for each VFS image sequence by a trained rater. U-Net, a well-established neural network architecture, was applied to the images. $70 \%$ of the frames were used to train, and the remaining $30 \%$ were used for validation. Full-image sequence predictions were also completed. The Dice coefficient was used to calculate similarity between the predicted and manual segments.

Result(s): On the validation set, the corresponding Dice coefficients were $0.95,0.76$, and 0.75 for $\mathrm{C} 2$ and $\mathrm{C} 4$, bolus, and hyoid. Across full-length image sequences for each swallow, the average and standard deviation of the Dice coefficient was $0.98 \pm 0.01$ for $\mathrm{C} 2$ and $\mathrm{C} 4$; $0.85 \pm 0.08$ for the bolus; and $0.91 \pm 0.01$ for the hyoid.

Conclusions (Including Clinical Relevance): The performance of this auto-segmentation algorithm with VFSS image sequences provides evidence that, with a small sample size, a neural network can learn underlying image characteristics to provide accurate anatomical segmentations. The dataset will be expanded to improve robustness of the network. Segments provide a foundation for future use of algorithms to automatically derive key VFSS measures (e.g., hyoid displacement, pharyngeal residue) that can be used to support clinical practice as well as analysis for research.

Relevant Financial Relationships: Nadeem Shaheen: Has affiliations to disclose;University of Wisconsin Madison:Salary/ Stipend:Employment I Rodolfo Peña-Chávez: Has affiliations to disclose;University of Wisconsin Madison:Salary/Stipend:Employment I Chris Ulmschneider: Has affiliations to disclose;University of Wisconsin Madison:Salary/Stipend:Employment I Ryan Burdick: Has affiliations to disclose;University of Wisconsin Madison:Salary/ Stipend:Employment I Joanne Yee: Has affiliations to disclose;William S. Middleton Veterans Affairs Hospital:Salary/ Stipend:Employment I Atsuko Kurosu: Has affiliations to disclose;National Institute of Health:Salary/ Stipend:Employment;University of Wisconsin Madison:Salary/ Stipend:Employment I Nicole Rogus-Pulia: Has affiliations to disclose;UW-Madison:Salary/Stipend:Employment;University Of Wisconsin Medical Foundation:Salary/Stipend:Employment;William S. Middleton Memorial Veterans Hospital:Salary/Stipend:Employment;National Institute of Health:Grant:Independent contractor (Including contracted research) | Bryan Bednarz: Has affiliations to disclose;University of Wisconsin Madison:Salary/ Stipend:Employment.

Relevant Non-financial Relationships: Nadeem Shaheen: Nothing to Disclose I Rodolfo Peña-Chávez: Has a Non-Financial Disclosure
Affliation;Dysphagia Research Society:Professional:Membership | Chris Ulmschneider: Nothing to Disclose I Ryan Burdick: Nothing to Disclose I Joanne Yee: Has a Non-Financial Disclosure Affliation;Dysphagia Research Society:Professional:Membership I Atsuko Kurosu: Has a Non-Financial Disclosure Affliation;Dysphagia Research Society:Professional:Membership I Nicole Rogus-Pulia: Has a Non-Financial Disclosure Affliation;Dysphagia Research Society:Professional:Board membership;Dysphagia Research Society:Professional:Membership | Bryan Bednarz: Nothing to Disclose.

\section{Respiratory-Swallow Coordination in a Rat Model of Chemoradiation}

Linda M. Rowe ${ }^{1,2}$, Michelle R. Ciucci ${ }^{1,2}$, Nadine Connor ${ }^{3,2}$, John Russell $^{1,2}$

Purpose: Safe deglutition manifests coordination of swallowing and respiration with a protective exhale-swallow-exhale pattern, which may be disrupted in people with head and neck cancer (HNC). Specifically, there are reports of post-swallow inhale events that elevate risk of penetration/aspiration. The effects of chemoradiation treatment (CRT) on the physiological mechanisms of swallow-respiratory phase relationships are not well understood. This gap in knowledge is largely due to challenges in isolating the specific effects of CRT versus other patient factors and the invasive nature of biological assays. The purpose of this study was to assess the validity of a rat model for investigating the effect of CRT on respiratory-swallow coordination to allow future studies correlating functional changes with underlying biology.

Method(s): Ten Sprague-Dawley rats received CRT (3 mg/kg Cisplatin, followed by 10 fractions of 4.5 Gy/day of radiotherapy to an $8 \mathrm{~mm} \times 12 \mathrm{~mm}$ area at the base of tongue). Ten additional control rats did not receive CRT. Videofluoroscopic swallow studies (VFSS) were performed at the 3-month time point in all rats. Diaphragm displacement was tracked as an indicator of respiratory phase. We examined the effect of CRT on swallow apnea durations, diaphragm movement, and bolus kinematics across the oropharyngeal (OP) and pharyngoesophageal (PE) phases of the swallow.

Result(s): Swallow-respiratory coordination patterns in all control rats were consistently characterized by exhalation after the swallow. However, CRT rats exhibited both post-swallow inhalation (CRT-IN) and post-swallow exhale (CRT-EX) patterns. The CRT-IN subgroup $(n=4)$ had significantly longer swallow apnea durations ( $p$ $\leq 0.0001$ ), faster bolus transit through the UESPE bolus speed $(p=0.014)$, initiated the swallow at lower diaphragm displacements $(p \leq 0.0001)$, and inhaled post-swallow to higher diaphragm displacements $(p \leq 0.0001)$ than the control group and CRT-EX subgroup. The CRT-EX subgroup $(n=6)$ took significantly smaller boluses than the control group $(p=0.039)$.

Conclusions (Including Clinical Relevance): The rat CRT model exhibits altered respiratory-swallow coordination consistent with clinical HNC findings. Simultaneous VFSS diaphragm and bolus tracking in rat CRT model are a useful and valid tool for examining functional swallowing outcomes, with underlying biological and physiological changes imposed by CRT in future studies.

Relevant Financial Relationships: Linda Rowe: Has affiliations to disclose;NIH:Grant:Employment I Michelle Ciucci: Has affiliations to disclose;NIH:Grant:Employment I Nadine Connor: Has affiliations to disclose;NIH:Grant:Employment I John Russell: Has affiliations to disclose;NIH:Grant:Employment.

Relevant Non-financial Relationships: Linda Rowe: Has a NonFinancial Disclosure Affliation;DRS:Professional:Membership;ASHA:Professional:Membership I Michelle Ciucci: Has a Non- 
Financial Disclosure Affliation;DRS:Professional:Membership;ASHA:Professional:Membership I Nadine Connor: Has a NonFinancial Disclosure Affliation;DRS:Professional:Board membership;ASHA:Professional:Membership | John Russell: Nothing to Disclose.

\section{Impact of Quantitative Objective Videofluoroscopic Swallowing Measures on Analysis and Treatment Recommendations in a Clinical Context}

\section{Gwen Kerrison $^{1,3}$, Anna Miles ${ }^{1}$, Michael Heron ${ }^{1,4}$, Jacqueline Allen $^{1,2}$}

Purpose: Although objective quantitative measures are available for videofluoroscopic swallow study (VFSS) interpretation, they are yet to be used routinely clinically. The aim of this study was to explore agreement between standard VFSS practice and quantitative analysis and the impact on diagnosis and recommendations.

Method(s): 100 adults referred for VFSS undertook a standardised VFSS protocol. Treating speech pathologists (SP), who had the full clinical history, used a traditional observational approach to report the study. Following this, two independent blinded raters analysed all VFSS quantitatively using timing and displacement measures. VFSS were also analysed using a traditional observational approach by one independent (non-treating) blinded rater. The study focused on diagnostic agreement across three constructs: pharyngeal constriction (PC), hyoid maximum displacement (Hmax) and pharyngoesophageal segment maximum opening (PESmax). Parameters were rated as within or outside 1SD of norms (Leonard and Kendall 2019). 50\% of VFSS were rated by an additional 11 independent blinded raters. Raters made binary judgements of normal vs impaired for the three constructs using frame-by-frame playback.

Result(s): PC, Hmax and PESmax achieved moderate agreement $(84 \%, 87 \%, 61 \%$, respectively) between traditional observational raters compared to excellent agreement $(100 \%$; ICC $=0.77-0.93)$ between quantitative raters. Incidence of reported impairment was lower in treating SPs and traditional observational raters compared with quantitative raters $(p<0.05)$, with the treating SP reporting $<50 \%$ of the impairments detected through quantitative measurement. For treating SPs, using quantitative measures as gold standard, sensitivity and specificity were variable (PC 37\%, 83\%; Hmax 26\%, 83\%; PESmax 37\%, 90\%, respectively). Rehabilitation exercises were recommended less often by treating SPs and traditional observational raters compared with quantitative raters.

Conclusions (Including Clinical Relevance): This study demonstrates that the introduction of quantitative measures for interpreting VFSS increased inter-rater agreement and accuracy of physiological impairment identification. This influenced rehabilitation recommendations with quantitative raters suggesting more targeted rehabilitation than those using a traditional observational approach. Quantitative measures warrant consideration in providing best evidence-based treatment recommendations.

Relevant Financial Relationships: Gwen Kerrison: Nothing to Disclose I Anna Miles: Nothing to Disclose I Michael Heron: Nothing to Disclose I Jacqueline Allen: Nothing to Disclose.

Relevant Non-financial Relationships: Gwen Kerrison: Nothing to Disclose I Anna Miles: Nothing to Disclose I Michael Heron: Nothing to Disclose I Jacqueline Allen: Nothing to Disclose.

\section{Impact of Expiratory Muscle Strength Training on Swallowing Kinematics in Individuals with ALS}

\author{
Lauren Tabor-Gray $^{2}$, Raele Robison ${ }^{1}$, Terrie Vasilopoulos ${ }^{3}$, \\ Emily Plowman ${ }^{1}$
}

Purpose: Expiratory muscle strength training (EMST) is an increasingly popular intervention utilized by clinicians in dysphagia rehabilitation. ${ }^{1}$ Although EMST is noted to improve subglottic pressure generation capacity crucial for effective cough production in neurodegenerative patient populations, ${ }^{2,3}$ a recent systematic review revealed the paucity of data investigating the impact of EMST on swallowing physiology. ${ }^{1}$ We previously reported that an 8-week EMST regimen led to improvements in maximum expiratory pressure (MEP) and cough in people with ALS (pALS)0. ${ }^{2}$ Motivated by this recent review, we aimed to examine the impact of EMST on swallowing physiology.

Method(s): 48 pALS were enrolled in a randomized sham-controlled trial and completed 8 weeks of either active-EMST $(n=24,50 \%$ MEP) or sham-EMST ( $n=24$, no load). Standardized videofluoroscopic swallowing evaluations $(40 \% \mathrm{w} / \mathrm{v}$ concentration Varibar@ cued, and self-administered) were completed pre- and postEMST. Analysis of Swallowing Physiology: Events, Kinematics \& Timing (ASPEKT) ${ }^{4}$ outcomes were performed on $3 \mathrm{cc}$ thin, $3 \mathrm{cc}$ pudding, and $20 \mathrm{cc}$ thin bolus trials by a blinded rater. Descriptives, $t$ tests, and repeated measures mixed models were performed $(p<0.05)$.

Result(s): Mean age was 61.4 (SD: 10.3), forced vital capacity was $79 \%$ (SD: 14.4), and 61\% were male. A total of 131 individual bolus clips were obtained. There were no overall differences in the pre-post change scores between active and sham groups for swallow reaction time $(p=0.67)$, LVC duration $(p=0.45)$, time to LVC $(p=0.20)$, pharyngeal transit time $(p=0.38)$, and pharyngeal area at maximum pharyngeal constriction $(p=0.20)$. There were no significant group differences between bolus types $(p>0.05)$.

Conclusions (Including Clinical Relevance): Although MEP and cough strength were impacted in this group post-EMST, no changes occurred in swallowing kinematics or pharyngeal constriction. These data suggest that EMST does not align with neuroplasticity principles of specificity and salience for dysphagia rehabilitation, but rather with the principle of transference to improve related functions of subglottic air pressure generation and airway defense physiologic capacity. ${ }^{5}$ This treatment modality, despite not directly impacting swallowing physiology, is particularly valuable in pALS given that they demonstrate compromised and eminent degradation in these related physiologic domains. Further work is needed to validate these findings.

Relevant Financial Relationships: Lauren Tabor-Gray: Has affiliations to disclose;ALS Association:Grant:Other Activities;Holy Cross Health:Salary/Stipend:Employment I Raele Robison: Nothing to Disclose I Terrie Vasilopoulos: Nothing to Disclose I Emily Plowman: Has affiliations to disclose;ALS Association:Grant:Other Activities;Biohaven:Consulting fee:Consulting;National Institute of Health:Grant:Other Activities.

Relevant Non-financial Relationships: Lauren Tabor-Gray: Has a Non-Financial Disclosure Affliation;DRS:Professional:Membership | Raele Robison: Has a Non-Financial Disclosure Affliation;DRS:Professional:Membership | Terrie Vasilopoulos: Nothing to Disclose | Emily Plowman: Has a Non-Financial Disclosure Affliation;DRS:Professional:Membership. 


\section{Feeding Skills and Eating Efficiency are Reduced in Self-feeding School-Age Children with Unilateral CP: An Unrecognized Reality}

\section{Georgia Malandraki ${ }^{1,2}$, Samantha Mitchell ${ }^{1}$, Barbara Brown ${ }^{1}$,} Rachel Hahn Arkenberg ${ }^{1}$, Jennifer Lundine ${ }^{3,4}$, Wendelin BurdoHartman $^{3,5}$, Bruce Craig ${ }^{6}$, Lisa Goffman ${ }^{7}$

Purpose: School-age children with unilateral cerebral palsy (UCP) are thought to have functional feeding and swallowing skills and rarely receive swallowing intervention. To determine if this preconceived notion should be challenged, we quantitatively examined the clinical feeding and swallowing skills and eating efficiency of children with UCP compared to those of typically developing children (TDC).

Method(s): Seventeen children with UCP (10 male; age range 7;2-12;2yoa, GMFCS levels range: I to II) and 17 age and sex-matched TDC participated. All children had typical cognition and none received services for feeding/swallowing issues at the time of participation. Children participated in a large-scale research project including a battery of behavioral and standardized swallowing, speech and language assessments, a surface electromyography evaluation, and an MRI scan. This abstract focuses on the behavioral swallowing data. Clinical feeding and swallowing skills were evaluated using the dysphagia disorders survey (DDS). Eating efficiency was assessed using a novel measure of mealtime efficiency (i.e., average sip/bite duration). Both assessments were completed during a standardized snack task including three consistencies (water, pudding, pretzel) and were video recorded for reliability. Group differences in average measure score were assessed via nonparametric bootstrap. Pearson correlation was used to assess the association between the measures. Result(s): Children with UCP demonstrated significantly higher (worse) total DDS scores (mean $=5.76$; SD: 3.48) than the TDC (mean: 1.53; SD: 0.63) (95\% CIs: 2.71, 5.94). Subscores related to oropharyngeal swallowing competencies primarily drove these differences. Average sip/bite duration was also significantly increased for the children with unilateral CP (mean: $13.23 \mathrm{~s}$; SD: 3.87) compared to the TDC (mean: $10.44 \mathrm{~s}$, SD: 1.88 ) (95\% CI $0.93,4.64$ ). Pearson correlations of 0.36 (UCP) and -0.11 (TDC) were not statistically significant.

Conclusions (Including Clinical Relevance): These findings reveal that both clinical feeding/swallowing skills and eating efficiency are reduced in self-feeding school-age children with UCP and suggest that these children's feeding and swallowing skills should be carefully evaluated as they may require treatment.

Relevant Financial Relationships: Georgia Malandraki: Has affiliations to disclose;Purdue University:Salary/ Stipend:Employment;NIH:Grant:Other Activities;Purdue Research Foundation:Grant:Other Activities;AACPDM:Grant:Other Activities | Samantha Mitchell: Has affiliations to disclose;Purdue University:Salary/Stipend:Employment;NIH:Salary/Stipend:Employment । Barbara Brown: Has affiliations to disclose;Purdue University:Salary/ Stipend:Employment;NIH:Salary/Stipend:Employment I Rachel Hahn Arkenberg: Has affiliations to disclose;Purdue University:Salary/ Stipend:Employment I Jennifer Lundine: Has affiliations to disclose;Ohio State University:Salary/ Stipend:Employment;AACPDM:Grant:Other Activities | Wendelin Burdo-Hartman: Has affiliations to disclose;Nationwide Children's Hospital:Salary/Stipend:Employment;AACPDM:Grant:Other Activities I Bruce Craig: Has affiliations to disclose;Purdue University:Salary/Stipend:Employment;NIH:Grant:Other Activities I Lisa Goffman: Has affiliations to disclose;University of Texas, Dallas:Salary/Stipend:Employment;NIH:Consulting fee:Consulting.

Relevant Non-financial Relationships: Georgia Malandraki: Has a Non-Financial Disclosure Affliation;DRS:Professional:Board
membership;ASHA:Professional:Membership;ASHA Journals:Professional:Volunteer membership on advisory committee or review panels;VA Merit Grants Panel:Professional:Volunteer membership on advisory committee or review panels;Sheppard Dysphagia Institute:Professional:Vounteer consulting;AACPDM:Professional:Membership | Samantha Mitchell: Has a Non-Financial Disclosure Affliation;Dysphagia Research Society:Professional:Membership;NSSLHA:Professional:Membership | Barbara Brown: Has a Non-Financial Disclosure Affliation;ASHA:Professional:Membership I Rachel Hahn Arkenberg: Has a Non-Financial Disclosure Affliation;Dysphagia Research Society:Professional:Membership;ASHA:Professional:Membership । Jennifer Lundine: Has a Non-Financial Disclosure Affliation;ASHA:Professional:Membership | Wendelin Burdo-Hartman: Has a Non-Financial Disclosure Affliation;Dysphagia Research Society:Professional:Membership;AACPDM:Professional:Membership I Bruce Craig: Nothing to Disclose I Lisa Goffman: Has a NonFinancial Disclosure Affliation;ASHA:Professional:Membership.

\section{Does Tongue Strength Influence Pharyngeal Swallow Outcomes? A Retrospective Analysis}

Erica G. Herzberg ${ }^{2,1}$, Sonja M. Molfenter ${ }^{1}$, Sofia Virzi $^{1}$, Sophia Chen $^{4}$, Matina Balou ${ }^{3}$

Purpose: Lingual resistance treatment protocols appear to consistently result in stronger lingual pressures, yet few studies examine the resulting impact on pharyngeal swallowing. Lingual function can be captured in multiple ways such as maximal strength, saliva swallow strength, or the difference between them (functional reserve). Our goal was to retrospectively explore the association between measures of lingual function and the expression of swallow safety and efficiency, while controlling for bolus and participant factors.

Method(s): Maximum anterior lingual strength, saliva swallow strength, and effortful swallow strength are captured with IOPI in triplicate per our standardized clinical evaluation (CE) protocol. Chart reviews were conducted on all outpatients (mixed etiology) seen between $12 / 18$ and $05 / 19(N=263)$. Patients seen for VFSS after $\mathrm{CE}$ were included $(N=98,48 \mathrm{M}$, mean age $=66) .2 \times$ IDDSI0 and $2 \times$ IDDSI- 4 boluses $(n=383)$ were blindly analyzed for safety (Penetration Aspiration Scale, PAS) and efficiency (Normalized Residue Ratio Scale vallecular, NRRSv and pyriforms, NRRSp). Regression models explored the influence of max anterior lingual strength, saliva swallow strength, effortful saliva swallow, functional reserve, age, sex, and viscosity on PAS, NRRSv and NRRSp. PAS was treated as a binary variable ( 1 and $2=$ safe, $\geq 3=$ unsafe).

Result(s): No measures of tongue strength significantly contributed to the expression of unsafe swallowing or residue. The final binary logistic regression for PAS $\left[\chi^{2}(6)=40.3\right.$, pseudo $\left.R^{2}=0.204\right]$ increased odds of unsafe swallows with thin liquid, males, and older age. The final linear regression for $\operatorname{NRRSv}[F(3,376)=13.5$, $p<0.001$, adj. $\left.R^{2}=0.10\right]$ predicted significantly more NRRSv for males and increased viscosity. The final linear regression model for NRRSp $\left[F(2,377)=18.8, p<0.001\right.$, adj $\left.R^{2}=0.09\right]$ predicted significantly more NRRSp for males and older age.

Conclusions (Including Clinical Relevance): Lingual strengthening is a popular intervention for patients with swallowing impairments; however, evidence supporting resulting improvements in pharyngeal swallow function is sparse. The current study failed to find an association between any measure of tongue strength and swallow safety or efficiency. This may call into question the rationale of lingual strengthening treatment to targeting pharyngeal swallowing deficits. Future prospective studies should explore pharyngeal physiological predictors of PAS and residue. 
Relevant Financial Relationships: Erica Herzberg: Has affiliations to disclose;NYU Langone Health:Salary/Stipend:Employment;NYU Steinhardt:Salary/Stipend:Employment I Sonja Molfenter: Has affiliations to disclose;NYU Steinhardt:Salary/Stipend:Employment I Sofia Virzi: Nothing to Disclose I Sophia Chen: Nothing to Disclose | Matina Balou: Has affiliations to disclose;NYU Langone Health:Salary/Stipend:Employment;NYU Grossman School of Medicine:Salary/Stipend:Employment.

Relevant Non-financial Relationships: Erica Herzberg: Nothing to Disclose I Sonja Molfenter: Has a Non-Financial Disclosure Affliation;DRS:Professional: Other volunteer activities I Sofia Virzi: Nothing to Disclose I Sophia Chen: Nothing to Disclose I Matina Balou: Has a Non-Financial Disclosure Affliation;DRS:Professional: Other volunteer activities.

\section{Defining Presbyphagia: Age-Related Changes in Healthy Swallowing}

\section{Renata Mancopes $^{1}$, Emily Barrett ${ }^{1}$, Pooja Gandhi ${ }^{1,2}$, Melanie Peladeau-Pigeon $^{1}$, Sana Smaoui ${ }^{1,2}$, Talia Wolkin ${ }^{1}$, Catriona M. Steele $^{1,2}$}

Purpose: Research suggests there are age-related changes in swallowing that do not constitute impairment ("presbyphagia"). Reference data exist for quantitative videofluoroscopic measures of swallowing in healthy adults under age 60 (Steele et al. 2019). It is unclear if these reference data can be generalized to older adults. The goal of this study was to explore the influence of age on quantitative measures of healthy swallowing, controlling for the effects of sex and sip volume, to determine the specific characteristics of presbyphagia. Method(s): Data were collected from a sex-balanced sample of 38 healthy adults over the age of 60 (range 61-82) and pooled with the under-60 reference data $(n=38)$ used by Steele et al. 2019. Each participant swallowed 3 naturally sized sips of $20 \% \mathrm{w} / \mathrm{v}$ thin barium. Blinded duplicate ratings of swallowing safety, efficiency, kinematics, and timing were made using the ASPEKT method. Hierarchical linear regression was used to determine the effects of age, sex, and sip volume on 16 parameters.

Result(s): There were no age-related changes in sip volume, number of swallows per bolus, frequency or severity of penetration-aspiration, duration of the hyoid-burst (HYB) to upper esophageal sphincter (UES) opening interval, time-to-laryngeal-vestibule-closure (LVC), hyoid peak position, hyoid speed, or residue. Significant changes seen with advanced age included longer swallow reaction time, UES opening duration, and LVC duration; larger pharyngeal area at rest (PhAR) and maximum constriction; and wider UES diameter. Male participants had larger sip volume and PhAR. Larger sip volumes were associated with multiple swallows per bolus and shorter HYBto-UES opening intervals.

Conclusions (Including Clinical Relevance): These results help to define presbyphagic changes in swallowing that can be expected in healthy older adults up to 80 years of age and distinguish them from changes that represent impairment. Certain parameters showed changes that were opposite in direction to changes that are usually considered to reflect impairment: longer UES opening, longer LVC duration, and wider UES opening. These changes may reflect possible compensations for slower bolus transit. Further research is needed to determine the points along the age continuum where observed agerelated changes in swallowing begin to emerge.

Relevant Financial Relationships: Renata Mancopes: Has affiliations to disclose;KITE - Toronto Rehabilitation Institute, University Health Network:Salary/Stipend:Employment I Emily Barrett: Has affiliations to disclose;KITE-Toronto Rehabilitation Institute, University Health Network:Salary/Stipend:Employment । Pooja
Gandhi: Has affiliations to disclose;KITE-Toronto Rehabilitation Institute, University Health Network:Salary/Stipend:Employment I Melanie Peladeau-Pigeon: Has affiliations to disclose;KITE-Toronto Rehabilitation Institute, University Health Network:Salary/ Stipend:Employment I Sana Smaoui: Has affiliations to disclose;KITE-Toronto Rehabilitation Institute, University Health Network:Salary/Stipend:Employment I Talia Wolkin: Has affiliations to disclose;KITE-Toronto Rehabilitation Institute, University Health Network:Salary/Stipend:Employment । Catriona Steele: Has affiliations to disclose;KITE-Toronto Rehabilitation Institute, University Health Network:Salary/Stipend:Employment;National Institute of Deafness and Other Communication Disorders:Grant:Independent contractor (Including contracted research);Nestle Health Science:Speaking fee:Teaching and speaking.

Relevant Non-financial Relationships: Renata Mancopes: Nothing to Disclose I Emily Barrett: Nothing to Disclose I Pooja Gandhi: Nothing to Disclose I Melanie Peladeau-Pigeon: Nothing to Disclose I Sana Smaoui: Nothing to Disclose I Talia Wolkin: Nothing to Disclose I Catriona Steele: Has a Non-Financial Disclosure Affliation;International Dysphagia Diet Standardisation Initiative:Professional:Board membership.

\section{Dysphagia in the Intensive Care: Multicentre, Binational Point Prevalence Survey}

\author{
Amy Freeman-Sanderson ${ }^{1,2,3}$, Bronwyn Hemsley ${ }^{1}$, Kelly \\ Thompson $^{3}$, Naomi Hammond ${ }^{3,4}$
}

Purpose: Dysphagia often occurs in an intensive care unit (ICU) patient cohort. Increased risk for dysphagia can result from the use of artificial airways and as a consequence of neurological and respiratory illnesses, all impacting on swallow function and safety. There is a lack of multisite data on the prevalence and management of dysphagia in ICUs. The aim of this study was to describe the prevalence of dysphagia in ICU patients and describe ICU level practices for diagnosis and management of dysphagia across Australia and New Zealand.

Method(s): The prospective observational epidemiology study was embedded within the Australian and New Zealand Intensive Care Society Clinical Trials Group and The George Institute for Global Health point prevalence program. Multisite ethical approval was granted for this study. The survey collected patient demographic information (e.g. age, sex), admission data (e.g. APACHE II), data on swallowing function (e.g. diagnosis of dysphagia, food/fluid levels, and health professional utilisation), and ICU unit swallowing management practices (e.g. protocols and training).

Result(s): A total of 44 ICUs participated with $452 / 626$ (72\%) participants not intubated and included in our study. Dysphagia was documented in $36(8 \%)$ participants, $61 \%$ female and mean age 60.3 years (SD 16.4). Mean APACHE II scores were similar in the dysphagia group compared to the non-dysphagia group (16.6 vs. 16.1; $95 \% \mathrm{CI}-2.78$ to 1.77 ). There was a significant difference in weight for patients with dysphagia $73.3 \mathrm{~kg}$ vs $82.1 \mathrm{~kg}$ compared to patients without dysphagia (95\% CI 0.43 to 17.07). Mean duration of ICU length of stay was 3.97 days longer ( $95 \% \mathrm{CI}-1.91$ to 9.86 ) and hospital length of stay 4.91 days longer (95\% CI -0.58 to 10.40 ) for patients with dysphagia. A protocol for management of swallowing was available in $20(45 \%)$ ICUs, swallowing resources in $17(39 \%)$ ICUs, and specific training for staff in 13 (30\%) ICUs.

Conclusions (Including Clinical Relevance): The prevalence of reported dysphagia was $8 \%$ on the study day, with those patients experiencing dysphagia having a lower body weight and more likely to be female. The majority of ICUs report an absence of protocols, training, and resources for managing dysphagia. Interdisciplinary 
collaboration of medical, nursing, and allied health teams, including speech language pathologists is needed to address the need for further guidance in the management of patients with dysphagia.

Relevant Financial Relationships: Amy Freeman-Sanderson: Nothing to Disclose I Bronwyn Hemsley: Nothing to Disclose I Kelly Thompson: Nothing to Disclose I Naomi Hammond: Nothing to Disclose.

Relevant Non-financial Relationships: Amy Freeman-Sanderson: Nothing to Disclose I Bronwyn Hemsley: Nothing to Disclose I Kelly Thompson: Nothing to Disclose I Naomi Hammond: Nothing to Disclose.

\section{Reliability of Dynamic Imaging Grade of Swallowing Toxicity (DIGEST) among Clinical Speech Pathologists: A Pilot Analysis}

\section{Carly E. Barbon ${ }^{1}$, Carla L. Warneke ${ }^{2}$, Clare P. Alvarez ${ }^{1}$, Katherine A. Hutcheson ${ }^{1}$}

Purpose: DIGEST is a validated method to grade the severity of pharyngeal dysphagia from the modified barium swallow (MBS) study based on safety and efficiency of bolus transfer. DIGEST is open source with evidence of early adoption by research and clinical users but is yet to have a formal training paradigm. Understanding reliability among clinical users is a necessary step to inform training for best use. The aim was to assess reliability as it relates to experience and training conditions in practicing clinicians.

Method(s): Eleven speech pathologists (SLPs) from a single academic institution participated as raters. Training factors included individual clinical DIGEST training, MBSImP certification, Board Certification in Swallowing Disorders (BCS-S), and years of experience. Raters were provided a standardized, triplicate lab-rated training set of MBS $(n=20)$ and a concealed folder for submission. Initial ratings (R1) were followed by a $2-4$ week break before rating a rekeyed MBS set (R2). Prevalence- and Bias-adjusted Kappa was used to estimate agreement and interpreted per Cohen's standard ( $k=0.81-1$ almost perfect, $0.61-0.80$ substantial, and $0.41-0.60$ moderate agreement)

Result(s): Experience of 11 SLPs ranged from 1 to 18 years (mean 6.7); 9 had prior clinical DIGEST training, 10 were MBSImP certified, and 0 BCS-S. Raters demonstrated median 80\% (range 74-95\%) exact agreement and substantial inter-rater reliability for overall DIGEST grade compared to the lab standard (median $k=0.75$, range $0.67-0.93) ; 45 \%$ of raters had almost perfect and $55 \%$ had substantial agreement. Median Safety (S) and Efficiency (E) agreement were substantial ( $k=0.72 ; k=0.63$, respectively). Exact agreement was median $80 \%$ (range $75-95 \%$ ) with substantial intra-rater agreement $(k=0.75)$ for $\mathrm{R} 1 \mathrm{v}$ lab-rated; $\mathrm{S}$ and $\mathrm{E}$ grade intra-rater agreement was almost perfect $(k=0.88)$ and substantial $(k=0.75)$, respectively. Inter-rater reliability decreased as years of experience increased $(r=-0.64, \mathrm{p}=0.04)$. No other factors significantly correlated with reliability.

Conclusions (Including Clinical Relevance): Clinical SLP raters with diverse training and experience using DIGEST demonstrate substantial to almost perfect reliability on overall DIGEST. Reliability for individual raters was more variable for safety and efficiency grades. Future studies should examine training paradigms to optimize reliability, with emphasis on efficiency as agreement for this domain was lowest.

Relevant Financial Relationships: Carly Barbon: Has affiliations to disclose; The University of Texas MD Anderson Cancer Center:Salary/Stipend:Employment I Carla Warneke: Has affiliations to
disclose;The University of Texas MD Anderson Cancer Center:Grant:Employment I Clare Alvarez: Has affiliations to disclose; The University of Texas MD Anderson Cancer Center:Salary/Stipend:Employment | Katherine Hutcheson: Has affiliations to disclose; The University of Texas MD Anderson Cancer Center:Salary/Stipend:Employment;NCI:Grant:Other Activities;NIDCR:Grant:Other Activities;PCORI:Grant:Other Activities; Thrive Foundation:Grant:Other Activities.

Relevant Non-financial Relationships: Carly Barbon: Nothing to Disclose I Carla Warneke: Nothing to Disclose I Clare Alvarez: Nothing to Disclose I Katherine Hutcheson: Nothing to Disclose.

Poster Presentation

\section{Concordance Between Patient-Reported Outcome Measures and Clinician-Rated Symptom Toxicity During and Following (Chemo)radiotherapy for Head and Neck Cancer}

\author{
Laurelie Wishart ${ }^{1,2}$, Laura Moroney ${ }^{2,4}$, Bena Brown ${ }^{1,2,3}$, \\ Elizabeth Ward $^{1,2}$, Clare Burns ${ }^{2,4}$, Jane Crombie ${ }^{4}$, Jennifer \\ Helios $^{3}$, Claire Blake ${ }^{5}$
}

Purpose: The use of patient-reported outcome measures (PROMs) in head/neck cancer (HNC) is increasingly well-recognised to improve patient-provider communication, identify supportive care needs in a timely manner, and improve quality of life and even survival. However, the congruence and reliability of PROM data as compared to clinician-rated objective assessment are still not well understood. The purpose of the current study was to investigate the relationship between patient-reported dysphagia and associated symptoms, and clinician-rated assessment during/following (chemo)radiotherapy ([C]RT) in patients with HNC.

Method(s): Institutional databases of HNC patients treated at two cancer centres in Brisbane, Australia (June 2016-December 2018) were retrospectively reviewed. One institution routinely collected weekly SLP-rated CTCAE, and the other collected patient-reported symptom data via a validated electronic PROM tool. Data from 626 patients $(n=208$ Institution $1, n=418$ Institution 2$)$ across seven parameters (dysphagia, odynophagia, mucositis, dysgeusia, dry mouth, thick saliva, nausea) during (Week 1-7) and 2 weeks post-(C)RT were triangulated. Ratings were collapsed to binary form (CTCAE-0-1 vs. 2-3; patient-reported symptoms nil/negligible vs. moderate/severe) and compared using Chi-square tests.

Result(s): Cohorts were statistically homogeneous with respect to demographics (age, gender, disease site, stage, treatment modality). Symptom trajectories over time were highly congruent between PROM/CTCAE ratings for all seven parameters, peaking in severity at week 7 . In regard to prevalence by week, dysphagia and dysgeusia demonstrated the most differences between PROM/CTCAE ratings, with significant $(p<0.01)$ differences observed at all time points for dysphagia, and all but one (Week 2) for dysgeusia. In contrast, remaining parameters were substantially more congruent between PROM/CTCAE, with no differences observed at all but two time spoints.

Conclusions (Including Clinical Relevance): While patients versus clinicians may perceive the severity of symptoms differently, overall, our findings demonstrated reasonable concordance between PROMs and clinician-rated dysphagia/associated sequelae in regard to symptom trajectory, confirming the clinical utility of PROMs to assist with the delivery of supportive care in this population. 
Relevant Financial Relationships: Laurelie Wishart: Has affiliations to disclose; Queensland Health:Salary/Stipend:Employment I Laura Moroney: Has affiliations to disclose;Queensland Health:Salary/ Stipend:Employment | Bena Brown: Has affiliations to disclose;Queensland Health:Salary/Stipend:Employment | Elizabeth Ward: Has affiliations to disclose;Queensland Health:Salary/ Stipend:Employment I Clare Burns: Has affiliations to disclose;Queensland Health:Salary/Stipend:Employment I Jane Crombie: Has affiliations to disclose;Queensland Health:Salary/Stipend:Employment I Jennifer Helios: Has affiliations to disclose; Queensland Health:Salary/Stipend:Employment I Claire Blake: Has affiliations to disclose; Queensland Health:Salary/Stipend:Employment.

Relevant Non-financial Relationships: Laurelie Wishart: Has a Non-Financial Disclosure Affliation;Dysphagia Research Society:Professional:Membership;Speech

Pathology

Australia:Professional:Membership | Laura Moroney: Nothing to Disclose I Bena Brown: Nothing to Disclose I Elizabeth Ward: Has a Non-Financial Disclosure Affliation;Dysphagia Research Society:Professional:Membership;Speech Pathology Australia:Professional:Membership | Clare Burns: Has a Non-Financial Disclosure Affliation;Dysphagia Researcj Society:Professional:Membership;Speech Pathology Australia:Professional:Membership | Jane Crombie: Nothing to Disclose I Jennifer Helios: Nothing to Disclose I Claire Blake: Nothing to Disclose.

\section{Translation, Cultural Adaptation and Validity Evidence of the Feeding/Swallowing Impact Survey (FS-IS) Instrument to Brazilian Portuguese}

\section{Deborah S. Levy ${ }^{1}$}

Purpose: The purpose of this study was to translate and adapt the Feeding/Swallowing Impact Survey (FS-IS) into Brazilian Portuguese and provide a validated instrument for caregivers of children with feeding/swallowing disorders.

Method(s): This cross-cultural study involved initial translation, synthesis of translations, back-translation, Committee of Experts and pre-test.

Result(s): The sample consisted of 95 primary caregivers of children with feeding/swallowing disorders classified by Pediatric Dysphagia Evaluation Protocol (PDEP) in mild, moderate-severe or profound dysphagia. Evidence of validity based on test content, response processes, internal structure and the relations to other variables was investigated. Internal consistency, test-retest, exploratory and confirmatory factor analysis were performed, in addition to the correlation with PedsQL ${ }^{\mathrm{TM}}$ FIM. The pre-test participants did not mention any difficulties in understanding the translated version. The Brazilian version Pt-Br-FS-IS presented Cronbach's Alpha of 0.83 , exploratory factor analysis verified that the instrument would not be unifactorial $(\mathrm{KMO}=0.74$ and Barllet's sphericity test $p<0.001)$ and confirmatory factor analysis confirmed the original model in three subscales with $X^{2} / \mathrm{df}=1.23$, $\mathrm{CFI}=0.92, \mathrm{TLI}=0.90$, RMSEA $(90 \% \mathrm{CI})=0.049(0.011-0.073)$ adjustment indexes and the ICC was excellent in all subscales and total score. The correlation with $\mathrm{PEdsQL}{ }^{\mathrm{TM}}$ was significant in the total score and subscales.

Conclusions (Including Clinical Relevance): This study successfully translated and cross-culturally adapted the FS-IS instrument to the Brazilian Portuguese language and the investigation of its validity evidence suggests that the Pt-Br-FS-IS is a reliable and valid tool to measure the impact of feeding/swallowing disorders on the quality of life of caregivers of affected children.
Relevant Financial Relationships: Deborah Levy: Nothing to Disclose.

Relevant Non-financial Relationships: Deborah Levy: Nothing to Disclose.

\section{Establishing Reference Values for Temporal Kinematic Swallow Events Across the Lifespan in Healthy Community Dwelling Adults Using HRCA}

\author{
Cara Donohue $^{1}$, Yassin Khalifa ${ }^{2}$, Shitong $\mathrm{Mao}^{2}$, Subashan \\ Perera $^{3}$, Ervin Sejdic ${ }^{2,4}$, James L. Coyle ${ }^{1,5}$
}

Purpose: Few studies have established reference values for temporal swallow kinematic events across the lifespan in healthy adults. Yet, determining normal variation in swallowing is vital for differentially diagnosing presbyphagia and dysphagia. High-resolution cervical auscultation (HRCA) is a noninvasive, sensor-based technology that uses acoustic signals from a contact microphone and vibratory signals from a tri-axial accelerometer to characterize swallowing using signal processing and machine learning techniques. HRCA signals have been used to annotate kinematic swallow events (e.g. duration of upper esophageal sphincter opening [DUESO], laryngeal vestibular closure [LVC]). This study aimed to determine 1. reference values for temporal swallow kinematic events; 2 . whether HRCA can accurately annotate kinematic swallow events in healthy adults. We hypothesized our reference values would closely match prior studies and that HRCA would accurately annotate kinematic swallow events in healthy adults.

Method(s): We completed kinematic analyses for 659 thin-liquid swallows from 70 healthy adults across the lifespan who underwent concurrent VFSSs and HRCA. We compared our swallow timing measures to a historical cohort of reference values for healthy thinliquid swallows (Steele et al. 2019; 114 swallows; 38 healthy adults) and used HRCA signals and machine learning algorithms to predict DUESO and LVC.

Result(s): There was no difference in swallow timing measures from our lab and the historical cohort except for LVC reaction time $(p<0.05)$, but small effect sizes were found for LVC duration and hyoid onset to UES opening and large effect sizes were found for swallow reaction time, LVC reaction time, and DUESO. Using HRCA signals alone, our machine learning algorithms annotated UES opening with $69.96 \%$ accuracy and UES closure with $64.52 \%$ accuracy within a 3 -frame error tolerance. Analysis is ongoing for $\mathrm{LVC}$ using HRCA signals and machine learning algorithms (76\% accuracy on a subset of data, thus, far).

Conclusions (Including Clinical Relevance): Overall, our reference values for swallow timing measures in healthy adults closely matched a historical cohort. This study provides preliminary evidence that HRCA can accurately annotate swallow kinematic events in healthy adults to determine dysphagia screening cutoffs. Future studies should replicate this work to establish a large database of swallows from healthy adults across the lifespan.

Relevant Financial Relationships: Cara Donohue: Has affiliations to disclose;University of Pittsburgh:Salary/Stipend:Employment;NIH:Salary/Stipend:Employment | Yassin Khalifa: Has affiliations to disclose;University of Pittsburgh:Salary/Stipend:Employment;NSF:Salary/Stipend:Employment I Shitong Mao: Has affiliations to disclose;University of Pittsburgh:Salary/Stipend:Employment;NIH:Salary/Stipend:Employment I Subashan Perera: Has affiliations to disclose;University of Pittsburgh:Salary/Stipend:Employment;NIH:Salary/Stipend:Employment | Ervin Sejdic: Has affiliations to disclose;University of Pittsburgh:Salary/ 
Stipend:Employment;NIH:Salary/Stipend:Employment;NSF:Salary/ Stipend:Employment I James Coyle: Has affiliations to disclose;University of Pittsburgh:Salary/ Stipend:Employment;NIH:Salary/Stipend:Employment;NSF:Salary/ Stipend:Employment.

Relevant Non-financial Relationships: Cara Donohue: Has a NonFinancial Disclosure Affliation;Dysphagia Research Society:Professional:Board membership;Dysphagia Research Society:Professional:Membership | Yassin Khalifa: Nothing to Disclose I Shitong Mao: Nothing to Disclose I Subashan Perera: Nothing to Disclose I Ervin Sejdic: Nothing to Disclose I James Coyle: Has a Non-Financial Disclosure Affliation;Dysphagia Research Society:Professional:Membership.

\section{Swallowing Pathophysiology in Individuals with ALS}

\section{Lauren Tabor-Gray ${ }^{1}$, Raele Robison ${ }^{2}$, Emily K. Plowman ${ }^{3}$}

Purpose: Dysphagia is reported to occur in $85 \%$ of people with ALS (pALS), with unsafe swallowing characterized by impairments in airway kinematics and timing (Waito 2020). We aimed to compare temporal and kinematic physiologic metrics of swallowing in pALS to established normative reference values and examine relationships between swallowing physiology and safety.

Method(s): 44 pALS underwent a standardized videofluoroscopic swallowing evaluation with cued and self-administered trials. ASPEKT outcomes of swallowing physiology and penetration aspiration scale scores were derived for $3 \mathrm{cc}$ thin, $20 \mathrm{cc}$ thin and $3 \mathrm{cc}$ pudding bolus trials (Steele et al. 2019). One sample $t$ tests (pALS vs. healthy reference data), Spearman rho' correlations (PAS and swallowing metrics), Chi squared and odds ratios were performed (alpha, $p<0.05)$.

Result(s): A total of 119 individual bolus clips were analyzed. Compared to healthy reference data, pALS demonstrated longer time to laryngeal vestibule closure (LVC, 251 vs. $176 \mathrm{~ms}$, $p<0.001$ ), shorter duration of LVC (398 vs. $436 \mathrm{~ms}, p<0.05$ ), longer pharyngeal transit time (PTT, 598 vs. $350 \mathrm{~ms}, p<0.001$ ), and a higher frequency of impairment in LVC (complete: $85 \%$, partial:13\%, incomplete:2\%). Swallow reaction time did not significantly differ between pALS and established normative values (SRT, 106 vs. $67 \mathrm{~ms}, p>0.05$ ). Swallowing safety was associated with time to LVC $(r=0.31, p=0.001)$, PTT $(r=0.19, p=0.03)$ and LVC movement integrity, $\chi^{2}(2)=11.1, p=0.004$. pALS with impaired LVC had a 4.8 higher odds of unsafe swallowing $(95 \% \mathrm{CI}$ $1.1,12.4)$.

Conclusions (Including Clinical Relevance): pALS demonstrated aberrant swallowing physiology characterized by longer PTT, longer time to LVC, shorter LVC and reduced LVC movement integrity. Unsafe swallowing was related to longer PTT, delayed time to LVC and impaired movement integrity of LVC. Identification of specific impairments in airway closure patterns and timing provide rationale for targeted dysphagia rehabilitation.

Relevant Financial Relationships: Lauren Tabor-Gray: Has affiliations to disclose;ALS Association:Grant:Other Activities;Holy Cross Health:Salary/Stipend:Employment | Raele Robison: Nothing to Disclose I Emily Plowman: Has affiliations to disclose;Univ of Florida:Salary/Stipend:Employment;Biohaven:Consulting fee:Consulting;ALS Association:Grant:Other Activities;National Institute of Health:Grant:Other Activities.

Relevant Non-financial Relationships: Lauren Tabor-Gray: Has a Non-Financial Disclosure Affliation;DRS:Professional:Membership | Raele Robison: Has a Non-Financial Disclosure Affliation;DRS:Professional:Membership | Emily Plowman: Has a Non-Financial Disclosure Affliation;DRS:Professional:Membership.

\section{Developing a Protocol for Objective Quantitative Analysis of Swallowing in Children: A Videofluoroscopic Study}

\author{
Isuru Dharmarathna ${ }^{1,3}$, Anna Miles ${ }^{2}$, Jacqueline Allen ${ }^{2}$
}

Purpose: Swallowing impairments in children can cause morbidity (malnutrition, failure to thrive, delayed growth) and mortality (compromised airway, aspiration pneumonia). Early and effective detection of swallowing impairments can lead to opportunities to intervene, thereby improving outcomes for children and reducing swallowing-related mortality and morbidity rates through proactive interventions. Objective quantitative measures in videofluoroscopic swallow studies (VFSS) can quantify swallow biomechanics. The literature contains a wide array of different measures, some measuring the same things despite different terminology resulting in difficulty in clinical application. There is a need for a feasible VFSS protocol including a few key time-effective and clinically relevant measures. In this study, we aimed to establish a standard protocol for objective quantitative VFSS analysis in children.

Method(s): Protocol development began with a systematic literature review to identify the objective quantitative swallow measures available in the literature. 20 objective quantitative and 9 descriptive measures were identified. Measures were tested for reliability and validity. A pediatric VFSS database of 553 children was collected using a standard protocol intended for objective analysis.

Result(s): Inter-rater and intra-rater reliability on measures obtained from VFSS database studies were satisfactory. Construct, criterion, content and external validity identified clinical relevance of the proposed protocol items. We explored validity of objective quantitative measures against key swallow risks such as aspiration and residue. During internal consistency testing, we removed measures based on Cronbach's alpha, when 2 or more measures were equivalent in measuring the same aspect of swallow biomechanics in children. At the end of the process, we present a VFSS protocol of reliable, valid and clinically relevant objective quantitative $(n=6)$ and descriptive measures $(n=3)$ with separate protocols for young infants ( $<9$ months) and older children.

Conclusions (Including Clinical Relevance): A standardized protocol for children helps optimize VFSS assessment, gathering the most information in order to understand underlying physiological manifestations causing swallowing impairments. This leads to more individualized treatment for children. Clinicians are encouraged to implement standard VFSS protocols with objective interpretation in children.

Relevant Financial Relationships: Isuru Dharmarathna: Nothing to Disclose I Anna Miles: Nothing to Disclose I Jacqueline Allen: Nothing to Disclose.

Relevant Non-financial Relationships: Isuru Dharmarathna: Nothing to Disclose I Anna Miles: Nothing to Disclose I Jacqueline Allen: Nothing to Disclose.

\section{Dysphagia and Intellectual Disability: Surveying the Practice Patterns of Clinicians in the Intermediate Care Facility (ICF) Setting}

\section{Melissa Farrow ${ }^{1}$}

Purpose: No studies have explored practice patterns of clinicians who manage dysphagia in those with intellectual disabilities (ID). Evidence from existing research indicates some uniformity in dysphagia management for heterogeneous patient populations. Yet it is 
unknown whether those with intellectual disability can be served in the same manner as other populations with respect to their swallowing disorders. The aim of this study was to identify the dysphagia assessment and treatment practices of clinicians working with those with ID who reside in ICF settings.

Method(s): Survey questions were reviewed for sensibility, content, format, and administration time by three independent SLPs with $>$ 10 years of clinical experience in dysphagia management. Feedback resulted in a final version of 39 questions. An email request to participate in an electronic survey was sent to 118 SLPs and OTs at 13 State Supported Living Centers (SSLC). Survey reliability was determined using a test-retest design and results were analyzed using descriptive and non-parametric statistics via SPSS software. Any licensed SLPs or OTs at the SSLCs who did not assess/treat dysphagia were excluded.

Result(s): Associations $(p \leq 0.05)$ between modifiers and reported practices behaviors were found. Most clinicians $(>80 \%)$ included current medical status, medical history, meal observations, and oral structures/functions in their assessments. Assessment barriers $(>45 \%)$ included cognitive deficits and behavioral challenges. Recommendations $(>40 \%)$ included diet modifications, adaptive feeding equipment, mealtime body positioning, and staff supervision. Treatment barriers $(>50 \%)$ were staff compliance, challenging behaviors, physical disabilities, and institutional practices.

Conclusions (Including Clinical Relevance): In the ICF setting, dysphagia is often managed through an interdisciplinary team approach that includes both speech pathologists and occupational therapists. Compensatory techniques rather than rehabilitative strategies are used to manage dysphagia in this population. ID and co-occurring behavioral disorders can limit clinical and instrumental assessment options. Barriers outside of the patient's medical status can also impede dysphagia treatment options within the ICF setting. Future research should investigate the effectiveness of using compensatory strategies such as diet modifications to improve the health and quality of life for those with ID and dysphagia.

Relevant Financial Relationships: Melissa Farrow: Nothing to Disclose.

Relevant Non-financial Relationships: Melissa Farrow: Nothing to Disclose.

\section{Central Control in Mental Practice During a Tongue Press Task: A Proof of Concept Study}

\section{Teresa Drulia ${ }^{1}$, Erin Kamarunas ${ }^{2}$, Sarah Szynkiewicz ${ }^{3}$, Lindsay Griffin $^{4,2}$, Morgann Henn ${ }^{2}$}

Purpose: Mental practice using motor imagery (MP-MI) is repeated mental rehearsal of a targeted movement without overt execution. Upper and lower extremity motor strength, as well as tongue pressures, are improved with repeated MP-MI, particularly when combined with physical exercise. There is limited understanding and continued debate regarding potential muscle activation during MP-MI influencing clinical outcomes. The purpose of this proof of concept study was to determine if muscle activation and task duration were different in MP-MI tongue presses compared to physical tongue presses.

Method(s): Participants included a typically aging cohort without swallowing or neurological impairments $(n=13$, mean age $=65.7)$. They completed 20 blocked trials of active exercise tongue presses and mental tongue presses in a counterbalanced order. Participants received training to the task, but durational cues were not provided during trials. Submental muscle activation during tasks was measured with surface electromyography (SEMG). Signals were filtered and normalized and then analyzed for amplitude (root mean square) and duration. Statistical comparisons were conducted with paired-samples $t$ tests.

Result(s): Mental tongue presses had significantly less submental muscle activation $(M=2.96 \%, \mathrm{SD}=2.49)$ compared to active tongue presses $(M=29.36 \%, \mathrm{SD}=10.24)$, a significant mean decrease of $26.40 \%, 95 \%$ CI $[20.25,32.55], t(12)=9.35, p<0.001, d=2.59$. There was no significant difference for task duration between mental tongue presses $(M=2.34 \mathrm{~s}, \mathrm{SD}=1.78)$ and active tongue presses $(M=2.34 \mathrm{~s}, \mathrm{SD}=1.54), 95 \%$ CI $[-0.45,0.45], t(12)=0.019$, $p=0.985, d=0.005$.

Conclusions (Including Clinical Relevance): Participants exhibited limited submental musculature contraction when completing MP-MI tongue presses compared to contraction observed during active exercise tongue presses. This supports the theory that any functional motor changes seen from treatment with MP-MI are likely from a central nervous system mechanism rather than from peripheral changes. Further, the task duration for both active tongue presses and MP-MI tongue presses was similar, suggesting an overlap of central motor planning for these two functions. While additional research is needed to determine the role of MP-MI in dysphagia management, it appears to be a promising mechanism for promoting central nervous system plasticity.

Relevant Financial Relationships: Teresa Drulia: Nothing to Disclose I Erin Kamarunas: Nothing to Disclose I Sarah Szynkiewicz: Nothing to Disclose I Lindsay Griffin: Nothing to Disclose I Morgann Henn: Nothing to Disclose.

Relevant Non-financial Relationships: Teresa Drulia: Nothing to Disclose I Erin Kamarunas: Nothing to Disclose I Sarah Szynkiewicz: Nothing to Disclose I Lindsay Griffin: Nothing to Disclose I Morgann Henn: Nothing to Disclose.

\section{Physiological Analysis of Swallowing Impairment Caused by Chronic Obstructive Pulmonary Disease (COPD) in Rats}

\section{Kouta Nagoya $^{1}$, Takanori Tsujimura ${ }^{1}$, Midori Yoshihara ${ }^{1}$, Makoto Inoue ${ }^{1}$}

Purpose: Chronic obstructive pulmonary disease (COPD) is the respiratory illness, of which the number of patients is expected to increase with age. Dyspnea and cough, sputum are known as the main symptom of COPD, often accompanied by swallowing impairment. Although many clinical reports dealing with swallowing impairment related to COPD have been published, physiological mechanism of swallowing changes due to COPD still remains unclear. So, we analyzed how COPD contributes to swallowing impairment using COPD rats.

Method(s): We produced COPD rats by intratracheal administration of elastase followed by LPS for SD rats. Data collection was performed at the point of 6 and 9 weeks after LPS administration. Examinations were carried out on control and COPD rats anesthetized with urethane $(1.3 \mathrm{~g} / \mathrm{kg})$. To identify swallowing and respiration, electromyographic (EMG) activity was recorded from digastric (Dig) and thyrohyoid (TH) muscles and diaphragm (Dia). Swallowing was evoked by superior laryngeal nerve (SLN) electrical stimulation.

Result(s): We confirmed COPD condition by micro-CT images and HE staining of lung. First, we analyzed respiration using Dia EMG activity. The respiratory rate of COPD rats did not show significant difference compared with control rats. Inspiratory duration of COPD rats was significantly longer, whereas expiratory duration was significantly shorter compared with control rats. The duty cycle (a ratio of the inspiratory duration to the total respiratory time) of COPD rats was significantly higher than that of control rats. Next, we analyzed swallowing changes. Swallowing elicited by SLN stimulation 
occurred at inspiration phase in almost all of COPD rats. The interval from inspiration starting to Dig activity occurring did not differ between control and COPD rats. At 9 weeks after LPS inhalation, the EMG active duration of TH was significantly longer compared with control rats of 6 weeks after LPS administration.

Conclusions (Including Clinical Relevance): Our study suggested that the discoordination of respiration due to COPD increased the frequency of swallowing reflex during inspiration. Furthermore, long term COPD affected the swallowing related muscle activity, especially $\mathrm{TH}$ muscle. This phenomenon may be related to the muscle atrophy originated from COPD. Thus, these physiological changes may cause a high risk of aspiration.

Relevant Financial Relationships: Kouta Nagoya: Nothing to Disclose I Takanori Tsujimura: Nothing to Disclose I Midori Yoshihara: Nothing to Disclose I Makoto Inoue: Nothing to Disclose.

Relevant Non-financial Relationships: Kouta Nagoya: Nothing to Disclose I Takanori Tsujimura: Nothing to Disclose I Midori Yoshihara: Nothing to Disclose I Makoto Inoue: Nothing to Disclose.

\section{Prophylactic Pharyngeal Strengthening Program in Healthy Older Adults: Preliminary Findings}

\section{Sonja M. Molfenter ${ }^{1}$, Erica Herzberg ${ }^{1,2}$, Danielle Brates ${ }^{1}$, Alexandra Soyfer ${ }^{3}$, Luis F. Riquelme ${ }^{3,4}$}

Purpose: Sarcopenia is the age-related loss of muscle strength and function. Our recent research has established that pharyngeal sarcopenia is associated with reductions in pharyngeal constriction and increases in pharyngeal residue and therefore may increase the risk for developing age-related dysphagia. The aim of this pilot study was to explore the impact of an 8-week prophylactic pharyngeal strengthening program on swallowing outcomes.

Method(s): 5 healthy older women (mean age 78.2, range 72-86) completed 8 weeks of pharyngeal exercises. Exercises were selected to target pharyngeal muscle activation and included effortful swallows, posterior tongue resistance, effortful pitch glides and tonguehold swallows. 10 repetitions of each exercise were completed per set, with sets gradually increasing from 2 in week 1 , to 3 in week 2 and to 4 in weeks 3-8. Sessions were conducted twice per week with a clinician with an additional 3 days per week home practice. Adherence was tracked with weekly logs. Outcome measures included measures of tongue strength (anterior maximum, posterior maximum, saliva swallow) using the Iowa Oral Performance Instrument, biomechanical and functional pharyngeal parameters on videofluoroscopy (pharyngeal constriction and normalized residue ratio scale (NRRS) on $2 x$ cup sip thin liquid per participant) and pharyngeal lumen volume from acoustic pharyngometry (representing pharyngeal sarcopenia). Given the pilot nature of this work and small sample size, trending results were reported.

Result(s): Paired $t$ tests revealed that post-exercise there were significant improvements in maximal anterior $(t=2.87, p=0.045)$ and posterior $(t=2.97, p=0.041)$ tongue strength, and trending increases in saliva swallow strength $(t=2.62, p=0.059)$ and pharyngeal volume $(t=2.21, p=0.09)$. Linear mixed effects regressions models revealed a significant improvement in vallecular residue $(F=6.26$, $p=0.025$ ) but not pyriform sinus residue or pharyngeal constriction. Conclusions (Including Clinical Relevance): Our results indicate that this 8 -week prophylactic pharyngeal exercise program is feasible and safe and appears to be effective in improving various parameters of swallowing function. Importantly these changes were observed in healthy individuals with functional swallowing, thus, supporting the initiation of a larger-scale trial with control conditions to definitively confirm the findings of this prophylactic exercise regimen.
Relevant Financial Relationships: Sonja Molfenter: Has affiliations to disclose;NYU:Salary/Stipend:Employment I Erica Herzberg: Has affiliations to disclose;NYU Langone:Salary/Stipend:Employment;NYU Steinhardt:Salary/Stipend:Employment I Danielle Brates: Nothing to Disclose I Alexandra Soyfer: Has affiliations to disclose;Barrique SLP, PC:Salary/Stipend:Employment I Luis Riquelme: Has affiliations to disclose;Barrique SLP, PC:Salary/Stipend:Employment;New York Medical College:Salary/Stipend:Employment.

Relevant Non-financial Relationships: Sonja Molfenter: Has a NonFinancial Disclosure Affliation;DRS:Professional: Other volunteer activities I Erica Herzberg: Nothing to Disclose I Danielle Brates: Nothing to Disclose I Alexandra Soyfer: Nothing to Disclose I Luis Riquelme: Nothing to Disclose.

\section{Early Reintroduction of Oral Feeding Following Surgery with Free Flap Reconstruction for Oral Cancer}

\author{
Grainne Brady $^{1}$, Lauren Leigh-Doyle ${ }^{1}$, Francesco Riva ${ }^{2}$, Cyrus \\ Kerawala $^{2}$, Justin Roe ${ }^{1,3,4}$
}

Purpose: People can remain nil by mouth for up to 12 days following surgery and free flap reconstruction for oral cancer. Timing of oral intake reintroduction is often dictated by surgeon preference to allow for adequate time for healing and to reduce the risk of flap dehiscence. An evaluation of the impact of early oral feeding after free flap reconstruction for oral cancer was undertaken.

Method(s): We retrospectively reviewed 29 consecutive patients, at a single, tertiary cancer centre, who underwent radical surgical resection with free flap reconstruction for oral cancer between January 2018 and December 2019. Data include the disease site and staging, type of flap reconstruction, duration of tracheostomy, duration of hospital stay, method and duration of enteral feeding, day of re-introduction of oral feeding, complications and Performance Status Scale for Head and Neck Cancer Normalcy of Diet (PSS-NOD) scores at point of discharge.

Result(s): The sample of patients included 17 males and 12 females. Average age was 59.5 years (range 24-88). Tumour sites included oral tongue $(n=10)$, maxilla $(n=6)$, mandible $(n=6)$ floor of mouth $(n=5)$ and buccal mucosa $(n=2)$. The majority of patients $(68 \%, n=19)$ had stage IV disease. One patient with high-grade sarcoma was also included. Flap reconstructions included anterolateral thigh $(n=11)$, fibular $(n=7)$, radial forearm $(n=6)$ and medial sural artery-free flap $(n=5)$. Tracheostomy was required for 11 patients with median time to decannulation at 7 days (range 3-20 days). The majority of patients were able to tolerate oral fluids on day one post operatively $(86 \% n=25)$. Nonoral feeding was required in $90 \%$ of patients $(n=26)$ including $45 \%$ who required gastrostomy placement $(n=13)$. Median days to nasogastric tube removal were 6 days (range 3-15 days). Median length of hospital stay was 10 days (range 3-51). Mean PSS-NOD score at point of hospital discharge was 36.55 (95\% CI 30.9-42.2). Flap failure was noted in one patient $(3 \% n=1)$.

Conclusions (Including Clinical Relevance): The adoption of early oral intake as part of enhanced recovery did not increase morbidity or adverse outcome. A potential shorter hospital stay may encourage the adoption of an early oral feeding protocol in patients who have free flap reconstructive surgery for oral cancer.

Relevant Financial Relationships: Grainne Brady: Nothing to Disclose I Lauren Leigh-Doyle: Nothing to Disclose I Francesco Riva: Nothing to Disclose I Cyrus Kerawala: Nothing to Disclose I Justin Roe: Nothing to Disclose.

Relevant Non-financial Relationships: Grainne Brady: Nothing to Disclose I Lauren Leigh-Doyle: Nothing to Disclose I Francesco Riva: 
Nothing to Disclose I Cyrus Kerawala: Nothing to Disclose I Justin Roe: Nothing to Disclose.

\section{Effect of Sarcopenia in Masticatory and Swallowing Muscles on Oropharyngeal Dysphagia in Patients with Acute Stroke}

Katsuhiko Sakai ${ }^{1}$, Masateru Katayama ${ }^{2}$, Yusuke Matsumoto ${ }^{3}$, Kentaro Honda $^{1}$, Nana Morita ${ }^{1}$, Junko Nakajima ${ }^{1}$, Dai Kamamoto $^{2}$, Sadao Suga ${ }^{2}$, Takeshi Nomura ${ }^{3}$, Nobuyuki Matsuura ${ }^{1}$

Purpose: Sarcopenia affects various outcomes of stroke patients, including oropharyngeal dysphagia (OD). Moreover, systemic skeletal muscle atrophy progresses after the onset of stroke, resulting in secondary sarcopenia known as stroke-related sarcopenia. However, the changes in masticatory and swallowing muscles that occur after the onset of stroke have not been studied. In the present study, we examined the changes in masticatory and swallowing muscles in patients with acute stroke and their effects on OD by determining the mass of masticatory and swallowing muscles using ultrasonography. Method(s): The subjects of the study were 24 patients (12 males and 12 females) with acute stroke. National Institutes of Health Stroke Scale (NIHSS) were collected as initial stroke severity. The swallowing function was assessed by the Functional Oral Intake Scale (FOIS) at the time of initial evaluation. The tongue thickness (TT) and the temporalis muscle thickness (TMT) were also measured using ultrasonography at the onset of stroke and 2 weeks after the initial evaluation. The OD in patients, defined by FOIS $\leqq 5$, and the rate of change of TT and TMT were examined.

Result(s): The average NIHSS at the onset of stroke was $9 \pm 8.7$. The FOIS assessment of their swallowing function identified 13 patients with OD. The average TT was $44.6 \pm 4.4 \mathrm{~mm}$ at the initial evaluation and $42.3 \pm 4.2 \mathrm{~mm} 2$ weeks after the initial evaluation, showing a significant decrease TT over time $(p<0.05)$. Further, the average TMT on the unaffected side was $6.0 \pm 1.8 \mathrm{~mm}$ at initial evaluation and $5.9 \pm 1.9 \mathrm{~mm} 2$ weeks after the initial evaluation, and that on the paralyzed side was $6.1 \pm 1.7 \mathrm{~mm}$ at the time of initial evaluation and $5.6 \pm 1.8 \mathrm{~mm} 2$ weeks after the initial evaluation, showing a decrease TMT over time, although there were not significant. The examination of the occurrence of OD and the rate of change in the mass of masticatory and swallowing muscles showed no significant difference.

Conclusions (Including Clinical Relevance): Stroke-related sarcopenia has been suggested to occur in masticatory and swallowing muscles. Therefore, appropriate exercise and nutritional management immediately after the onset of stroke are critical. The present study found no association between the progression of masticatory and swallowing muscle atrophy and OD. However, such atrophy may be associated with long-term swallowing function, and its extended examination is needed.

Relevant Financial Relationships: Katsuhiko Sakai: Nothing to Disclose I Masateru Katayama: Nothing to Disclose I Yusuke Matsumoto: Nothing to Disclose I Kentaro Honda: Nothing to Disclose I Nana Morita: Nothing to Disclose I Junko Nakajima: Nothing to Disclose I Dai Kamamoto: Nothing to Disclose I Sadao Suga: Nothing to Disclose | Takeshi Nomura: Nothing to Disclose | Nobuyuki Matsuura: Nothing to Disclose.

Relevant Non-financial Relationships: Katsuhiko Sakai: Nothing to Disclose I Masateru Katayama: Nothing to Disclose I Yusuke Matsumoto: Nothing to Disclose I Kentaro Honda: Nothing to Disclose I Nana Morita: Nothing to Disclose I Junko Nakajima: Nothing to Disclose I Dai Kamamoto: Nothing to Disclose I Sadao Suga: Nothing to Disclose | Takeshi Nomura: Nothing to Disclose | Nobuyuki Matsuura: Nothing to Disclose.

\section{Impact of Reducing Fluoroscopy Pulse Rate on Adult- Modified Barium Swallow Studies}

\author{
Heather Bonilha $^{1}$, Erin Reedy ${ }^{1}$, Janina Wilmskoetter ${ }^{1}$, Paul \\ Nietert $^{1}$, Bonnie Martin-Harris ${ }^{2}$
}

Purpose: Reducing the X-ray pulse rate during modified barium swallow studies (MBSSs) is a common method used with the intent of minimizing radiation exposure. However, when the pulse rate is reduced, the temporal resolution is also reduced. The reduction in temporal resolution poises a significant risk to diagnostic accuracy as it limits the number of unique images captured from each swallow. Many aspects of swallowing are brief and, therefore, unlikely to be visualized during MBSS where pulse rates of 15 or less are used. We hypothesized that the reduction of temporal resolution, associated with reducing the pulse rate, impairs clinician judgments of clinically meaningful aspects of swallowing physiology.

Method(s): We evaluated the impact of reducing pulse rate from 30 pulses per second (pps) to 15, 7.5, and 4pps in 200 MBSSs from adult patients. Speech-language pathologists scored the modified barium swallow impairment profile components individually using the research scoring protocol. We also assessed how the impact of pulse rate varied by trial type (bolus consistency and presentation).

Result(s): Reducing pulse rate had a robust impact on scores of the initiation of pharyngeal swallow, laryngeal elevation, epiglottic movement, and pharyngeal stripping wave $(p<0.05)$. Other statistically significant findings related to the impact of reduced pulse rate on scores of bolus transport/lingual motion, oral residue, soft palate elevation, tongue base retraction, and pharyngeal residue $(p<0.05)$. The impact of pulse rate on the evaluation of swallowing physiology was observed across all bolus types: thin, nectar, honey, pudding, and cookie $(p<0.05)$. The most robust results were noted for thin and nectar thick boluses as well as single-cup sips and sequential liquid swallowing tasks.

Conclusions (Including Clinical Relevance): We have definitively demonstrated that reducing the pulse rate impacts the evaluation of key aspects of swallowing physiology. We have also demonstrated that this impact occurs across bolus types, and therefore, pulse rate should not be reduced any trial type during an MBSS. These results, paired with our knowledge of the low cancer risks associated with MBSSs in adults, supports the clinical use of continuous or 30pps.

Relevant Financial Relationships: Heather Bonilha: Has affiliations to disclose;Medical University of South Carolina:Salary/ Stipend:Employment;NIH:Consulting fee:Membership on advisory committee or review panels;NIH:Grant:Other Activities I Erin Reedy: Has affiliations to disclose;Medical University of South Carolina:Salary/Stipend:Employment I Janina Wilmskoetter: Has affiliations to disclose;Medical University of South Carolina:Salary/ Stipend:Employment;NIH:Grant:Other Activities I Paul Nietert: Has affiliations to disclose;Medical University of South Carolina:Salary/ Stipend:Employment;NIH:Grant:Other Activities I Bonnie MartinHarris: Has affiliations to disclose;Northwestern University:Salary/ Stipend:Employment;Veteran's University:Grant:Other Activities;NIH:Grant:Other Activities;Northern Speech Services:Royalty:Other Activities;Northern Speech Service:Speaking fee:Teaching and speaking;MUSC Foundation for Research Development:Royalty:Other Activities;US Provisional Patent US 62/710, 324: Hold Patent on equipment:Other Activities;Bracco Diagnostics, Inc:Grant:Other Activities. 
Relevant Non-financial Relationships: Heather Bonilha: Has a NonFinancial Disclosure Affliation;Dysphagia Research Society:Professional:Volunteer membership on advisory committee or review panels;ASHA Journals:Professional:Volunteer membership on advisory committee or review panels;ASHfoundation:Professional:Volunteer membership on advisory committee or review panels I Erin Reedy: Nothing to Disclose I Janina Wilmskoetter: Nothing to Disclose I Paul Nietert: Nothing to Disclose I Bonnie Martin-Harris: Has a Non-Financial Disclosure Affliation;DRS:Professional:

Membership;ASHA:Professional:Membership.

\section{The Effect of Automated Milk Delivery on Feeding Performance in Infants}

Khaled Adjerid $^{1}$, Christopher J. Mayerl ${ }^{\mathbf{1}}$, Francois D. Gould ${ }^{\mathbf{1}, 2}$, Chloe E. Edmonds ${ }^{1}$, Kendall E. Steer ${ }^{1}$, Laura E. Bond ${ }^{1}$, Rebecca Z. German ${ }^{1}$

Purpose: Milk acquisition for infant feeding, usually through a maternal breast or bottle and nipple, consists of several coordinated and potentially rhythmic behaviors, including sucking, bolus transport, and swallowing. Previously, the automated delivery of milk at different frequencies and volumes has been shown to alter swallow physiology. However, previous work did not fully decouple variation in delivery frequency and volume from variation in overall flow rate, nor compare to bottle feeding. Using a validated infant pig animal model, we hypothesized that infant feeding behavior and performance are improved with automatic feeding input as compared with bottle feeding.

Method(s): We recorded drinking in infant pigs using high-speed VFSS (100 fps) while delivering infant milk replacer formula mixed with barium. We delivered milk replacer at a constant volume flow rate of $1.2 \mathrm{ml} / \mathrm{s}$, with 5 different combinations of rate $(2-6 \mathrm{~Hz})$ and aliquot volume $(0.2$ to $0.6 \mathrm{ml})$ in addition to bottle feeding. We measured suck frequency, 2D swallow bolus size, total pharyngeal transit time, and swallow frequency.

Result(s): We found no impact of delivery frequency or aliquot volume on any feeding parameter, so we then grouped automated feeding together for statistical analyses with bottle feeding. We found that bottle feeding resulted in higher suck frequency (bottle $=4.41 \pm 0.90$ suck/second vs. automated feeder $=3.7 \pm 0.43$ suck/second), smaller boluses (bottle $=36.52 \pm 14.99 \mathrm{~mm}^{2}$ vs. automated feeder $=78.36 \pm 29.72 \mathrm{~mm}^{2}$ ), and lower milk transport time $($ bottle $=180 \pm 28 \mathrm{~ms}$ vs. automated feeder $=230 \pm 44 \mathrm{~ms}$ ) than on the automated feeder.

Conclusions (Including Clinical Relevance): Since sucks are used to acquire milk aliquots in bottle feeding, it is likely that bottle sucking yields a greater degree of infant control in milk acquisition, as evidenced by the generally lower variation observed in bolus size and transit time. Although sucking was observed in automated feeding, the reduced bolus transit time and swallow frequencies as well as the higher variation in sucking frequency in bottle feeding suggest that the efficiency of sucking in infants is a rate limiting step in the formation of boluses and drinking frequency.

Relevant Financial Relationships: Khaled Adjerid: Nothing to Disclose I Christopher Mayerl: Nothing to Disclose I Francois Gould: Nothing to Disclose I Chloe Edmonds: Nothing to Disclose I Kendall Steer: Nothing to Disclose I Laura Bond: Nothing to Disclose I Rebecca German: Nothing to Disclose.

Relevant Non-financial Relationships: Khaled Adjerid: Nothing to Disclose I Christopher Mayerl: Nothing to Disclose I Francois Gould: Nothing to Disclose I Chloe Edmonds: Nothing to Disclose I Kendall Steer: Nothing to Disclose I Laura Bond: Nothing to Disclose I Rebecca German: Nothing to Disclose.

\section{Identifying Risk of Malnutrition in Dysphagic Head and Neck Cancer Patients}

Sorina R. Simon ${ }^{1,2,3}$, Walmari Pilz ${ }^{1,7}$, Frank J. Hoebers ${ }^{3,4}$, Irene P. Leeters ${ }^{1}$, Annemie M. Schols ${ }^{5}$, Rianne A. Willemsen ${ }^{3,5,6}$, Bjorn Winkens $^{8,9}$, Laura W. Baijens ${ }^{1,3}$

Purpose: Malnutrition in head and neck cancer (HNC) patients is associated with increased morbidity and mortality. The purpose of this study is twofold: to identify the risk of malnutrition in a subpopulation of patients with oropharyngeal dysphagia (OD) secondary to $\mathrm{HNC}$, and to determine the relationship between the risk of malnutrition versus tumor characteristics, treatment modality, time interval between the end of oncological treatment and swallowing assessment date, functional oral intake scale (FOIS), body mass index (BMI), aspiration, pharyngeal pooling, and OD-related quality of life (QoL).

Method(s): Patients with OD secondary to HNC who visited the outpatient clinic for OD were enrolled in the study. The Short Nutritional Assessment Questionnaire (SNAQ) was used to screen patients for the risk of malnutrition, and consists of questions on unintentional weight loss, decreased appetite, and the need for an adjusted diet including supplemental drinks or tube feeding. Patients underwent a swallowing examination protocol including a standardized endoscopic evaluation of swallowing and QoL questionnaires. Logistic regression analysis was performed adjusted for age, gender, and tumor classification if appropriate.

Result(s): Seventy-five dysphagic HNC patients were included. Forty-eight percent $(N=36)$ of the patients presented a high risk of malnutrition using SNAQ. The majority of the patients $(81.3 \%$; $N=61$ ) was on a total oral diet (FOIS 4-7). Moreover, BMI did not appear to be a reliable screening for malnutrition as a normal BMI was often associated with an increased risk of malnutrition. In contrast, patients who were underweight or overweight did not show an association with a high risk of malnutrition. With the exception of BMI, no other variables studied such as treatment modality, FOIS, etc. were found to be associated with the risk of malnutrition.

Conclusions (Including Clinical Relevance): This study emphasizes the importance of early nutritional screening in dysphagic HNC patients, as almost half of these patients presented a high risk of malnutrition. The SNAQ screening tool may identify HNC patients with OD that need to be referred to a dietician for additional nutritional assessment and nutritional support even when their BMI is within normal range.

Relevant Financial Relationships: Sorina Simon: Nothing to Disclose I Walmari Pilz: Nothing to Disclose I Frank Hoebers: Nothing to Disclose | Irene Leeters: Nothing to Disclose | Annemie Schols: Nothing to Disclose I Rianne Willemsen: Nothing to Disclose I Bjorn Winkens: Nothing to Disclose I Laura Baijens: Nothing to Disclose. Relevant Non-financial Relationships: Sorina Simon: Nothing to Disclose | Walmari Pilz: Nothing to Disclose I Frank Hoebers: Nothing to Disclose I Irene Leeters: Nothing to Disclose I Annemie Schols: Nothing to Disclose I Rianne Willemsen: Nothing to Disclose I Bjorn Winkens: Nothing to Disclose I Laura Baijens: Nothing to Disclose.

\section{Validity and Reliability of DIGEST-FEES}

Heather Starmer $^{1}$, Loni Arrese ${ }^{2}$, Lauren Tabor-Gray ${ }^{6}$, Susan Langmore $^{3}$, Joe Murray ${ }^{4}$, Joanne Patterson ${ }^{5}$, Jessica Pisegna ${ }^{3}$, Justin Roe $^{7}$, Katherine A. Hutcheson ${ }^{8}$

Purpose: The Dynamic Imaging Grade of Swallowing Toxicity $\left(\right.$ DIGEST $^{\mathrm{TM}}$ ) was developed to grade pharyngeal dysphagia severity 
as measured by videofluoroscopic swallowing studies as a toxicity endpoint in oncology, according to the NCI's Common Terminology Criteria for Adverse Events framework. As fiberoptic endoscopic evaluation of swallowing (FEES) is also commonly used to assess swallowing in the head and neck cancer population, a comparable scale is needed. DIGEST was adapted into DIGEST-FEES as previously presented. This investigation sought to evaluate the validity and reliability of the DIGEST-FEES scale.

Method(s): Three blinded speech-language pathologists were trained in use of the DIGEST-FEES scale and demonstrated at least $80 \%$ concordance with a gold standard rater. To determine inter $(n=100$ FEES) and intra-rater reliability ( $n=32$ FEES), FEES examinations from an existing database of patients treated for head and neck cancer were independently rated by all three raters using the DIGEST-FEES criteria. Validity was established by comparing overall DIGESTFEES scores with MD Anderson Dysphagia Inventory (MDADI) scores, Functional Oral Intake Scores (FOIS), Secretion Severity Scores (SSS), and Yale Pharyngeal Residue Rating scores for vallecula and pyriform sinuses by Spearman's correlation $(p<0.05)$. Intraclass correlations were calculated to determine inter-rater and intra-rater reliability.

Result(s): Inter-rater reliability was judged to be good for safety scores (0.87 95\% CI 0.82-0.90), efficiency scores (0.75 95\% CI $0.62-0.83)$, and overall DIGEST grade (0.83 95\% CI 0.75-0.88). Intra-rater reliability was excellent $(0.9-0.91)$. DIGEST-FEES was significantly associated with all criterion measures including MDADI $(r=-0.434, p<0.0001)$, FOIS $(r=-0.433, p<0.0001)$, SSS $(r=0.469, p<0.0001)$, Yale Vallecula $(r=0.733, p<0.0001)$, and Yale Pyriform Sinus ratings $(r=0.652, p<0.0001)$.

Conclusions (Including Clinical Relevance): DIGEST-FEES is a reliable instrument to describe the severity of safety, efficiency, and overall swallowing impairment in patients with head and neck cancer. Consistent with the original DIGEST scale, strong correlation is noted between DIGEST-FEES and the Yale Pharyngeal Residue Severity Scale and moderate for measures of swallowing-related quality of life, diet level, and secretion severity.

Relevant Financial Relationships: Heather Starmer: Nothing to Disclose I Loni Arrese: Nothing to Disclose I Lauren Tabor-Gray: Nothing to Disclose I Susan Langmore: Nothing to Disclose I Joe Murray: Nothing to Disclose I Joanne Patterson: Nothing to Disclose I Jessica Pisegna: Nothing to Disclose I Justin Roe: Nothing to Disclose I Katherine Hutcheson: Nothing to Disclose.

Relevant Non-financial Relationships: Heather Starmer: Nothing to Disclose I Loni Arrese: Nothing to Disclose I Lauren Tabor-Gray: Nothing to Disclose I Susan Langmore: Nothing to Disclose I Joe Murray: Nothing to Disclose I Joanne Patterson: Nothing to Disclose I Jessica Pisegna: Nothing to Disclose I Justin Roe: Nothing to Disclose I Katherine Hutcheson: Nothing to Disclose.

\section{Speech-Language Pathologists' Knowledge, Attitudes, and Experience of Obstructive Sleep Apnea}

\section{Emma Wallace $^{1}$, Ankita Bhutada ${ }^{2}$, Kendrea Garand ${ }^{2}$, William Broughton $^{3}$, Danny Eckert ${ }^{1}$}

Purpose: Obstructive Sleep Apnea (OSA) is a common sleep-related breathing disorder characterized by repeated narrowing or closure of the upper airway during sleep. Despite growing evidence that dysphagia is a frequent sequalae of OSA, the role of speech-language pathologists (SLPs) in the management of OSA remains unclear. The aim of this international survey study was to evaluate SLPs knowledge, attitudes, and experience of OSA.

Method(s): A validated questionnaire, Obstructive Sleep Apnea Knowledge and Attitudes (OSAKA), was distributed to SLPs internationally via an online survey. Additional information on demographics, educational history, and clinical practices was ascertained. Descriptive and inferential statistics were calculated for all variables of interests.

Result(s): From a total of 1647 respondents, 822 clinicians from twenty-four countries completed all survey questions and were included in the final analysis. Knowledge of OSA among SLPs was limited. The mean (SD) of correctly answered questions on the knowledge component of the OSAKA questionnaire was 52\% $(19.4 \%)$. The majority of SLPs (91\%) agreed that identifying patients with OSA was important. Over half $(n=418)$ of SLPs reported having patients with OSA on their caseload, with majority referred for dysphagia services (69\%). However, only half of SLPs reported confidence in their ability to assess or manage dysphagia in patients with OSA.

Conclusions (Including Clinical Relevance): Although SLPs are involved in the management of patients with OSA, they are practicing with limited knowledge and education of the disorder. These findings highlight the need to increase education and training related to OSA for SLPs. In addition, there is a need for targeted research to increase the evidence base and inform development of clinical practice guidelines for SLPs to manage OSA as part of a multidisciplinary approach.

Relevant Financial Relationships: Emma Wallace: Nothing to Disclose I Ankita Bhutada: Nothing to Disclose I Kendrea Garand: Nothing to Disclose | William Broughton: Nothing to Disclose | Danny Eckert: Nothing to Disclose.

Relevant Non-financial Relationships: Emma Wallace: Nothing to Disclose I Ankita Bhutada: Nothing to Disclose I Kendrea Garand: Nothing to Disclose I William Broughton: Nothing to Disclose I Danny Eckert: Nothing to Disclose.

\section{Inter-rater Reliability, Parent Perception and Clinician Satisfaction with Bottle Feeding Assessments Conducted Via Telepractice}

\author{
Madeline K. Raatz $^{1,2}$, Elizabeth Ward ${ }^{2,3}$, Jeanne Marshall ${ }^{1,2}$, \\ Clare Burns $^{2,4}$
}

Purpose: A telepractice model for conducting synchronous (realtime) bottle feeding assessments for infants has been proposed, but reliability has not yet been established. This study aimed to examine the inter-rater reliability of bottle feeding assessments conducted via telepractice in patient homes, as well as consumer perceptions and clinician satisfaction with this mode of service delivery.

Method(s): Children with bottle feeding difficulties were assessed in their homes by two speech language pathologists (SLPs) who were randomly assigned to either the (a) telepractice condition (online) or (b) in-person condition (at child's home). The simultaneous assessment was led by the telepractice SLP. A purpose-built assessment form guided evaluation of general development, state, colour, respiration, oral motor function (including oral reflex and non-nutritive suck assessment), positioning, bottle feeding, overall feeding skills and feeding recommendations. Parent perception of telepractice was recorded pre and post appointment, and clinician satisfaction was collected after the appointment.

Result(s): Thirty-one children (1 month-2 years) were assessed via telepractice (13 initial/17 follow up appointments). High levels of agreement $(>80 \%)$ between the online and in-person SLP were reached for all assessment elements. Overall, parents' perceptions of bottle-feeding assessments conducted via telepractice improved following their appointment ( $n=25$ questionnaires returned), with $96 \%$ of parents reporting that they were comfortable having their child's feeding assessed online. The online assessment was reported to be 
comparable to an in-person assessment $(76 \%)$ and the telepractice assessment reportedly accurately represented their child's feeding skills $(92 \%)$. Clinicians reported that telepractice was an effective service delivery method for $90 \%$ of bottle feeding appointments and that they would re-offer telepractice services to $93 \%$ of children based on their session.

Conclusions (Including Clinical Relevance): Bottle feeding assessments conducted via telepractice produced comparable clinical results to in-person assessment. Clinicians and parents were satisfied with the telepractice service delivery model. Results support the potential of this service model to facilitate access to feeding services for families who prefer to receive care via telepractice, or when limitations to in-person care exist.

Relevant Financial Relationships: Madeline Raatz: Has affiliations to disclose;Queensland Health:Salary/Stipend:Employment;The Children's Hospital Foundation:Grant:Other Activities | Elizabeth Ward: Has affiliations to disclose;Queensland Health:Salary/ Stipend:Employment I Jeanne Marshall: Has affiliations to disclose;Queensland Health:Salary/Stipend:Employment I Clare Burns: Has affiliations to disclose;Queensland Health:Salary/ Stipend:Employment.

Relevant Non-financial Relationships: Madeline Raatz: Has a NonFinancial Disclosure Affliation;Dysphagia Research Society:Professional:Membership;Speech

Pathology

Australia:Professional:Membership I Elizabeth Ward: Has a Non-Financial Disclosure Affliation;Speech Pathology Australia:Professional:Membership;Dysphagia Research Society:Professional:Membership | Jeanne Marshall: Has a Non-Financial Disclosure Affliation;Speech Pathology Australia:Professional:Membership;Dysphagia Research Society:Professional:Membership I Clare Burns: Has a Non-Financial Disclosure Affliation;Speech Pathology Australia:Professional:Membership;Dysphagia

Research Society:Professional:Membership.

\section{Characterizing Effortful Swallows from Healthy Community Dwelling Adults Across the Lifespan Using HRCA Signals and MBSImP Scores}

\section{Cara Donohue $^{1}$, Yassin Khalifa ${ }^{2}$, Subashan Perera ${ }^{3}$, Ervin Sejdic $^{2,5}$, James L. Coyle ${ }^{1,4}$}

Purpose: There is high demand for inexpensive, non-invasive, portable tools that can accurately measure swallow function and determine appropriate treatment plans for patients with dysphagia. High-resolution cervical auscultation (HRCA) is a non-invasive sensor-based technology that uses acoustic and vibratory signals combined with advanced signal processing and machine learning techniques to quantify swallowing physiology. HRCA has determined swallowing safety (penetration-aspiration scale), annotated swallow kinematic events (e.g., hyoid bone displacement, laryngeal vestibule closure, duration of upper esophageal sphincter opening), and differentiated between swallows from healthy people and swallows from various patient populations with a high degree of accuracy. This study aimed to determine 1 . Whether HRCA can differentiate between noneffortful and effortful swallows; 2 . Whether there are differences in MBSImP components (\#9, \#11, \#14) between non-effortful and effortful swallows. We hypothesized that HRCA would classify noneffortful and effortful swallows with a high degree of accuracy and that there would be differences in MBSImP scores between swallows. Method(s): We analyzed 247 thin-liquid swallows from 36 healthy adults (mean age $65.53 \pm 7.67$ years) who underwent standardized VFSSs with concurrent HRCA at University of Pittsburgh Medical Center. We used multiple machine learning classifiers that use HRCA signal features to classify swallows as non-effortful or effortful and compared MBSImP component scores (\#9, \#11, \#14) between noneffortful and effortful swallows with a linear mixed model.

Result(s): There was a statistically significant $(p<0.05)$ difference in nine HRCA signal features between the non-effortful and effortful swallows. Using HRCA signal features alone, decision trees, and linear discriminant analysis classified swallows with up to $74 \%$ accuracy and an average area under curve of $61.2 \%$. There were no differences in MBSImP component scores (\#9, \#11, \#14) between non-effortful and effortful swallows $(p>0.05)$.

Conclusions (Including Clinical Relevance): This preliminary study demonstrates HRCA's potential as a biofeedback method for dysphagia treatment. Future studies should expand upon these findings by including a larger number of swallows from patients with dysphagia in addition to healthy adults and by investigating other treatment exercises that would benefit from biofeedback (e.g., Mendelsohn maneuver).

Relevant Financial Relationships: Cara Donohue: Has affiliations to disclose;NIH:Salary/Stipend:Employment;University of Pittsburgh:Salary/Stipend:Employment I Yassin Khalifa: Has affiliations to disclose;NSF:Salary/Stipend:Employment;University of Pittsburgh:Salary/Stipend:Employment I Subashan Perera: Has affiliations to disclose;University of Pittsburgh:Salary/Stipend:Employment;NIH:Salary/Stipend:Employment I Ervin Sejdic: Has affiliations to disclose;University of Pittsburgh:Salary/Stipend:Employment;NIH:Salary/Stipend:Employment;NSF:Salary/Stipend:Employment | James Coyle: Has affiliations to disclose;University of Pittsburgh:Salary/Stipend:Employment;NIH:Salary/Stipend:Employment;NSF:Salary/Stipend:Employment.

Relevant Non-financial Relationships: Cara Donohue: Has a NonFinancial Disclosure Affliation;Dysphagia Research Society:Professional:Board membership;Dysphagia Research Society:Professional:Membership | Yassin Khalifa: Nothing to Disclose I Subashan Perera: Nothing to Disclose I Ervin Sejdic: Nothing to Disclose I James Coyle: Has a Non-Financial Disclosure Affliation;Dysphagia Research Society:Professional:Membership.

\section{Exercise in Patients with amyotrophic lateral sclerosis: An Unsuccessful Meta-analysis}

Cara Donohue ${ }^{1}$, Mary Catherine Cazalas ${ }^{2}$, Ryan J. Colquhoun ${ }^{3}$, David Lacomis $^{4}$, Kendrea Garand ${ }^{2}$, Giselle Carnaby

Purpose: Amyotrophic lateral sclerosis (ALS) leads to debilitating impairments in mobility, respiratory, and bulbar functioning due to upper and lower motor neuron degeneration. Research in animal and human models provides promising evidence that exercise (e.g., aerobic, respiratory muscle strength training [RMST]) may slow disease progression while strenuous exercise may lead to faster deterioration. Exercise in patients with ALS (PALS) remains controversial in clinical and research settings due to conflicting research evidence to date. We attempted to perform a meta-analysis to determine effects of exercise in PALS.

Method(s): Four electronic databases (CINAHL, Scopus, PubMed, Cochrane) and a manual search were used to retrieve articles from database inception to December 2019. Index terms included: ALS OR amyotrophic lateral sclerosis OR motor neuron disease OR Lou Gehrig's disease AND exercise OR remedial exercise OR exercise therapy OR strength OR resistance training OR range of motion. Criteria for article inclusion: full-text article published in English and exercise-based intervention study involving human subjects diagnosed with ALS/motor neuron disease. Studies were screened using Covidence (Cochrane tool) and followed Preferred Reporting Items for Systematic Reviews and Meta-Analyses (PRISMA) statement standards. Two authors rated level of evidence with the Oxford Centre 
for Evidence-based Medicine Levels of Evidence and study quality using the QualSyst critical appraisal tool.

Result(s): 24 (of 959) studies were included. Varying exercise regimens and treatment dosages were observed. Such heterogeneity precluded meta-analyses from being performed on each intervention. Most studies $(N=20 ; 83 \%)$ reported beneficial impacts of exercise (e.g., maintenance and/or improvements in function), suggesting its role in prolonging survival and/or improving quality of life in PALS. Specifically, RMST demonstrated potential in prolonging pulmonary and swallow function. While these results are promising, study limitations (e.g., sample sizes, variability of exercise prescription, and high attrition) hinder enthusiasm.

Conclusions (Including Clinical Relevance): This systematic review supports that exercises, including RMST, are safe, well tolerated, and potentially beneficial for PALS. However, there is a need for further research to bolster and expand upon current findings.

Relevant Financial Relationships: Cara Donohue: Nothing to Disclose I Mary Catherine Cazalas: Nothing to Disclose I Ryan Colquhoun: Nothing to Disclose I David Lacomis: Nothing to Disclose I Kendrea Garand: Nothing to Disclose I Giselle Carnaby: No Answer.

Relevant Non-financial Relationships: Cara Donohue: Has a NonFinancial Disclosure Affliation;Dysphagia Research Society:Professional:Board membership;Dysphagia Research Society:Professional:Membership I Mary Catherine Cazalas: Nothing to Disclose I Ryan Colquhoun: Nothing to Disclose I David Lacomis: Nothing to Disclose I Kendrea Garand: Has a Non-Financial Disclosure Affliation;Dysphagia Research Society:Professional:Board membership;Dysphagia Research Society:Professional:Membership I Giselle Carnaby: No Answer.

\section{Effects of Caloric Density on Infant Formulas and Their Anti-Reflux Correlates According to IDDSI Methodology}

\section{Abigail M. Spoden ${ }^{3}$, Abbey Sterkowitz ${ }^{3}$, Anna Maunu ${ }^{3}$, Kayla Hernandez $^{1}$, Memorie Gosa ${ }^{2}$, Katlyn E. McGrattan ${ }^{3}$}

\section{Purpose:}

Background: Provision of thickened liquids is a commonly used treatment for infants with dysphagia. Despite its beneficial treatment effect, immaturity of the infant gut prevents utilization of many commercially available thickeners. This investigation tested the ability to achieve thickened liquids with modified caloric densities and anti-reflux formulas.

Method(s): Methods: Enfamil Infant, Similac Advance, and their anti-reflux correlates, Enfamil A.R. and Similac Spit-Up, were used. Formulas were tested in their ready to feed and powder $20 \mathrm{kcal} / \mathrm{oz}$ variants. Powder formulas were mixed with graduated caloric densities ranging from 20 to $30 \mathrm{kcal} / \mathrm{oz}$. Formula thickness was evaluated using the IDDSI Flow Test. Each condition underwent flow testing every $5 \mathrm{~min}$ for $30 \mathrm{~min}$ to simulate the duration of a typical bottle feed. As many caregivers make and refrigerate formula in batches and warm them for feeds, liquids were tested after $3 \mathrm{~h}$ of refrigeration.

Result(s): Results: Standard formulas remained thin liquids across all caloric densities. Examination of anti-reflux formula revealed Enfamil A.R. ready to feed was slightly thick; however, in its $20 \mathrm{kcal} /$ oz powder formulation, this thickness was not met until 15 min after mixing. Increasing Enfamil A.R. caloric density resulted in stepwise increases in thickness, while Similac Spit-Up remained a thin liquid under all conditions. Specifically, Enfamil A.R. mixed at $24 \mathrm{kcal} / \mathrm{oz}$ was classified as slightly thick immediately after testing versus the thin-liquid designation at the $22 \mathrm{kcal} / \mathrm{oz}$ regimen. Likewise, though it started out as slightly thick when mixed at $26 \mathrm{kcal} / \mathrm{oz}$ formulation, it increased to mildly thick at the end of $30 \mathrm{~min}$. Similar observations were observed at 28 and $30 \mathrm{kcal} / \mathrm{oz}$ formularies, which changed from mild to moderately thick designations. Enfamil A.R. and Similac SpitUp both increased thickness after $3 \mathrm{~h}$ of refrigeration and thinned with subsequent warming.

\section{Conclusions (Including Clinical Relevance):}

Conclusions: These results demonstrate the potential for Enfamil A.R. to be utilized as a thickener for dysphagia management. Despite its anti-reflux qualification, Similac Spit-Up was not found to be a viable option as it did not progress beyond thin designation. Future investigations examining nutritional and gastric safety of this methodology are warranted before clinical application.

Relevant Financial Relationships: Abigail Spoden: Has affiliations to disclose;Undergraduate Research Opportunities Program, University of Minnesota-Twin Cities:Grant:Other Activities I Abbey Sterkowitz: Has affiliations to disclose;Undergraduate Research Opportunities Program, University of Minnesota-Twin Cities:Grant:Other Activities I Anna Maunu: Has affiliations to disclose;Undergraduate Research Opportunities Program, University of Minnesota-Twin Cities:Grant:Other Activities I Kayla Hernandez: Has affiliations to disclose;Boston Children's Hospital:Salary/ Stipend:Employment । Memorie Gosa: Has affiliations to disclose;University of Alabama:Salary/Stipend:Employment;NSF, 1852161:Grant:Other Activities I Katlyn McGrattan: Has affiliations to disclose;University of Minnesota:Salary/Stipend:Employment;Masonic Children's Hospital:Salary/Stipend:Employment;Dr. Brown's Medical:Speaking fee:Other Activities;Biogen:Consulting fee:Consulting;Avexis:Consulting fee:Consulting;Biogen, US-SPN19-11520:Grant:Independent contractor (Including contracted research);NIH, NICHD, 1R41HD104305-01:Grant:Other Activities;nuBorn Medical:Ownership interest(e.g., stocks, stock options or other ownership interest excluding diversified mutual funds):Ownership;Science Stand:Ownership interest(e.g., stocks, stock options or other ownership interest excluding diversified mutual funds): Ownership.

Relevant Non-financial Relationships: Abigail Spoden: Nothing to Disclose | Abbey Sterkowitz: Nothing to Disclose | Anna Maunu: Nothing to Disclose I Kayla Hernandez: Nothing to Disclose I Memorie Gosa: Has a Non-Financial Disclosure Affliation;ASHA Pediatric Normal and Disordered Feeding \& Swallowing Topic Committee:Professional:Membership I Katlyn McGrattan: Has a NonFinancial Disclosure Affliation;DRS Membership Committee:Professional:Membership;ASHA Pediatric Normal and Disordered Feeding \& Swallowing Topic Committee:Professional:Membership.

\section{Lingual Pressure Impairment Thresholds for Unsafe and Inefficient Swallowing in ALS}

\section{Raele Robison $^{1,2,3}$, Lauren DiBiase ${ }^{2}$, Amy Ashley ${ }^{1,2}$, Kasey McElheny $^{2}$, Amber Anderson', James Wymer ${ }^{4}$, Emily K. Plowman 1,2,4,5}

Purpose: Reduced lingual strength is reported in people with ALS (pALS) that is associated with unsafe and inefficient swallowing. In limb motor and autonomic systems, critical reserve depletion thresholds have been identified that once reached give rise to impairment in subserving functions. It is currently unclear if a critical depletion threshold exists for lingual strength and swallowing function. We, therefore, aimed to identify lingual pressure depletion thresholds (LDT) for impairments in swallowing safety and efficiency in pALS.

Method(s): Thirty pALS attended a single testing session and completed a standardized videofluoroscopic swallowing examination and lingual pressure testing using the Iowa Oral Performance Instrument 
to index maximum isometric anterior lingual pressure. Duplicate blinded ratings of the Penetration Aspiration Scale (PAS) and Analysis of Swallowing Physiology: Events, Kinematics and Timing (ASPEKT) were performed. Binary classifications of safety (unsafe: PAS: $\geq 3$ ) and efficiency (inefficient: $\geq 3 \%$ total pharyngeal residue) were derived. Descriptives and receiver-operating characteristic curve analyses (AUC, sensitivity, specificity) were performed in SPSS $($ alpha $=0.05)$.

Result(s): LDT demonstrated a superior discriminant ability to identify swallowing safety impairment (AUC: 87) compared to swallowing efficiency impairment (AUC: 77).

A LDT of $37 \mathrm{kPa}$ optimized sensitivity (91\%) and specificity (68\%) for identification of unsafe swallowing, correctly classifying safety status in $87 \%$ of pALS (AUC $0.87, p=0.001$ ). A LDT of $46 \mathrm{kPa}$ optimized sensitivity (86\%) and specificity (56\%) to identify inefficient swallowing, correctly classifying efficiency status in $77 \%$ of pALS (AUC $=0.77, p=0.02$ ).

Conclusions (Including Clinical Relevance):

In this group of pALS, the lingual strength depletion threshold was higher for impairments in swallowing efficiency versus swallowing safety. These cross-sectional data indicate that smaller reductions to lingual pressure capacity affected swallowing efficiency and may suggest a temporal evolution regarding emergence of functional impairment type in pALS. Future work utilizing a longitudinal study design is warranted to further explore this preliminary finding.

Relevant Financial Relationships: Raele Robison: Nothing to Disclose I Lauren DiBiase: Nothing to Disclose I Amy Ashley: Nothing to Disclose I Kasey McElheny: Nothing to Disclose I Amber Anderson: Nothing to Disclose | James Wymer: Has affiliations to disclose;University of Florida:Salary/Stipend:Employment I Emily Plowman: Has affiliations to disclose;University of Florida:Salary/ Stipend:Employment;Biohaven:Consulting fee:Consulting;National Institutes of Health:Grant:Other Activities;ALS Association:Grant:Other Activities.

Relevant Non-financial Relationships: Raele Robison: Has a NonFinancial Disclosure Affliation;Dysphagia Research Society:Professional:Membership | Lauren DiBiase: Nothing to Disclose | Amy Ashley: Nothing to Disclose I Kasey McElheny: Nothing to Disclose I Amber Anderson: Nothing to Disclose I James Wymer: Nothing to Disclose I Emily Plowman: Has a Non-Financial Disclosure Affliation;Dysphagia Research Society:Professional:Membership.

\section{Effects of Varying Transcutaneous Electrical Stimulation Pulse Duration on Hyolaryngeal Kinematics in Healthy Adults}

\footnotetext{
Ali Barikroo ${ }^{1}$

Purpose: Limited research in swallowing physiology has suggested that using submental transcutaneous electrical stimulation (TES) with short pulse duration (PD) $(300 \mu \mathrm{s})$ may enhance the impact on deep extrinsic tongue muscles, thereby pulling the tongue down during swallowing. However, it was unclear whether the same TES protocol can have a differential impact on hyolaryngeal kinematics. This study aimed to compare the effect of submental TES with varying PDs on anterior and superior hyolaryngeal kinematics at rest and during swallowing in healthy adults.

Method(s): Twenty-four healthy adults (22-77 years of age) participated in this study. Anterior and superior hyolaryngeal excursion measures were collected using videofluoroscopic swallowing study. Each subject swallowed three trials of $10 \mathrm{ml}$ pudding under three TES conditions: no stimulation, short PD $(300 \mu \mathrm{s})$, and long PD (700 $\mu \mathrm{s})$. TES was delivered using two-channel surface electrodes on the submental area. In both short and long PD conditions, TES amplitude
}

was gradually increased until participants reached their maximum tolerance level. Videofluoroscopic data were analyzed using VideoPad Video Editor and Image J programs. One-way repeated measure ANOVAs were conducted to identify within-subject effect of TES condition.

Result(s): For hyoid movement, short PD selectively placed the hyoid bone on a more anterior position at rest and reduced anterior hyoid excursion during swallowing when compared with no TES condition. Regarding laryngeal movement, both TES protocols put the larynx on a more anterior position at rest and reduced anterior laryngeal excursions during swallowing when compared with no TES condition. Varying PDs had no significant effect on the superior hyoid and laryngeal movements at rest and during swallowing.

Conclusions (Including Clinical Relevance): Although both TES protocols demonstrated a comparable impact on reducing anterior laryngeal excursions, the TES protocol with short PD had an enhanced effect on reducing anterior hyoid excursion during swallowing. This reduced range of motion results from stimulating the deep submental muscles, which primarily place the hyoid and larynx to a more forward position before swallowing onset. Overall, the TES protocol with short PD may have an increased benefit in facilitating swallowing in patients with dysphagia.

Relevant Financial Relationships: Ali Barikroo: Has affiliations to disclose;Kent State University:Salary/Stipend:Employment.

Relevant Non-financial Relationships: Ali Barikroo: Has a NonFinancial Disclosure Affliation;Dysphagia Research Sociaety:Professional:Membership.

\section{Therapeutic Interventions for Neonates Who Are Bottle Fed: A Scoping Review}

\section{Raquel Garcia ${ }^{1}$}

Purpose: The purpose of this poster presentation is to examine the efficacy and validity of commonly used feeding interventions used with premature infants in the Neonatal Intensive Care Unit (NICU). Method(s): The investigators' primary outcome was to determine the efficacy of the use of therapeutic interventions for neonates who are bottle fed. We completed a scoping review of the scientific literature using SCOPUS electronic database. We used the following search terms: premat* and/or feed, premature and/or feed, preterm* and/or feed. This search yielding 14, 982 articles was first screened based on title. Then articles were narrowed down by their titles, resulting in 6609 articles. We removed 122 duplicate articles, resulting in 6487 articles to be screened by their abstracts. After review of the article abstracts, an additional 6305 articles were excluded secondary to abstract not referencing prematurity nor feeding. This yielded 182 articles for full-text review for inclusion criteria. Seven articles were added via forward and backward searching of each article selected, yielding 189 articles.

Result(s): Findings from this scoping review revealed that there are many feeding interventions used with neonates that are subjective. Inherent assumptions about neonatal feeding characteristics have prompted hypervigilance on improving bolus expression, volume consumption, physiologic stability, safety, and endurance at the bedside. Flow rate, pacing, and chin/cheek support did not have scientific evidence to substantiate its efficacy. Across studies, we found that neonates who were provided feeding techniques fed the same or worse than the control group. Essentially, we found that the neonates underlying prematurity and corrected age had increased influence over feeding competencies regardless of the frequency or dosage of the mentioned feeding interventions.

Conclusions (Including Clinical Relevance): We found that there was minimal scientific evidence to support the use of direct feeding 
interventions in bottle-fed neonates. In sum, the available evidence suggests limited treatment effects. The effects of direct feeding interventions, in bottle-fed neonates, needs further rigorous research. Further work should include prospective longitudinal randomized controlled trials that assess the role of the direct feeding intervention across infancy, beyond the time in the neonatal intensive care unit. Relevant Financial Relationships: RAQUEL GARCIA: Nothing to Disclose.

Relevant Non-financial Relationships: RAQUEL GARCIA: Nothing to Disclose.

\section{Implementation and Evaluation of a Speech Language Pathology VFSS Referring Model}

\author{
Shana T. Taubert ${ }^{1,2}$, Clare Burns ${ }^{1,2}$, Elizabeth Ward $^{2,3}$, Lynell \\ Bassett $^{1}$
}

Purpose: Speech language pathologists (SLPs) routinely refer patients for videofluoroscopic swallow studies (VFSS) to inform dysphagia intervention. However, in many countries, only doctors are authorised to complete medical imaging request forms due to radiation safety legislation. This process of seeking a medical referral can impact workflow and patient access to VFSS. The aim of this study was to evaluate the efficiency and safety of an alternate SLP-led VFSS referring model, compared to medical referring.

Method(s): Implementing the new SLP-led referring model required establishment of a Medical Imaging Internal Request Protocol and training four SLPs as VFSS referrers. Over a 3-month period preimplementation, and during a 6-month implementation period, data were prospectively collected on (1) timeliness of form completion, (2) any delay in VFSS appointments, and (3) compliance with radiation safety standards for information on request forms. Post-implementation, stakeholders (SLPs and doctors) were interviewed, with data analysed using the Consolidated Framework for Implementation Research to determine implementation barriers, facilitators and sustainability factors.

Result(s): Timeliness of form completion pre-implementation (3months; $n=61$ ) versus the SLP-led model implementation period (6months; $n=112)$ was comparable $(p>0.05)$. Compliance with radiation safety standards was significantly higher in the SLP-led model $(100 \%)$ than pre-implementation $(49 \% ; p<0.0001)$. Interviews revealed SLPs and doctors were very positive about the model. SLPs reported the purpose-built training equipped them well for the referring role. Factors facilitating implementation were the SLP-led model aided safe VFSS referrals; the SLP skill-set was compatible with completing VFSS request forms; strong communication networks between SLPs and doctors; and continued engagement of stakeholders throughout implementation. The availability of trained SLP referrers, ongoing stakeholder education, and outcome monitoring were critical sustainability factors.

Conclusions (Including Clinical Relevance): The SLP-led VFSS referring model is efficient and well received by stakeholders. SLPs demonstrated excellent compliance with radiation safety requirements. Having a structured protocol and SLP training procedure supported implementation. Ensuring staff have timely access to SLP referrers and ongoing monitoring are essential for sustainability.

Relevant Financial Relationships: Shana Taubert: Has affiliations to disclose;Queensland Health:Salary/Stipend:Employment;Royal Brisbane Hospital Foundation:Grant:Other Activities I Clare Burns: Has affiliations to disclose;Queensland Health:Salary/Stipend:Employment I Elizabeth Ward: Has affiliations to disclose;The University of Queensland:Salary/Stipend:Employment;Queensland Health:Salary/ Stipend:Employment I Lynell Bassett: Has affiliations to disclose;Queensland Health:Salary/Stipend:Employment.
Relevant Non-financial Relationships: Shana Taubert: Has a NonFinancial Disclosure Affliation;Speech Pathology Australia:Professional:Membership | Clare Burns: Has a Non-Financial Disclosure Affliation;DRS:Professional:Membership;Speech Pathology Australia:Professional:Membership | Elizabeth Ward: Has a NonFinancial Disclosure Affliation;DRS:Professional:Membership;Speech Pathology Australia:Professional:Membership I Lynell Bassett: Has a Non-Financial Disclosure Affliation;Speech Pathology Australia:Professional:Membership.

\section{Examining the Influence of Respiratory-Swallow Coordination on Penetration-Aspiration in Parkinson's Disease}

\section{$\underline{\text { James A. Curtis }}{ }^{1}$, Brianna Kiefer ${ }^{1}$, Michelle S. Troche ${ }^{1}$}

Purpose: Respiratory-swallow coordination (RSC) is thought to be critical for safe and efficient swallowing. Suboptimal RSC has been reported in Parkinson's disease (PD) and is characterized, in part, by prolonged respiratory pauses, and by swallows without the exhaleswallow-exhale pattern. The aims of this study were (1) to examine if RSC changes as a function of swallowing tasks in PD and (2) to assess the influence of suboptimal RSC on penetration-aspiration in PD.

Method(s): People with PD were recruited to undergo simultaneous RSC and FEES assessments. During the FEES, participants were presented with 23 boluses across 11 swallowing tasks. RSC and penetration-aspiration were examined for each swallow. RSC measures included (1) respiratory phase patterning, which was coded as either 'exhale-swallow-exhale' or 'not exhale-swallow-exhale'; and (2) respiratory pause duration, measured in seconds. Penetrationaspiration was also analyzed using the PAS. Mixed modeling analyses were used to determine if RSC changed across swallowing tasks, and to determine if the likelihood of penetration-aspiration increased with suboptimal RSC.

Result(s): Twenty-four participants were recruited. Most participants were early to mid-stage PD, with $25 \%$ considered to be advanced stage PD. On average, participants exhibited the optimal exhaleswallow-exhale for $30.2 \%$ of swallows (range 6.0-69.7\%) with an average respiratory pause duration of $3.55 \mathrm{~s}(\mathrm{SD}=1.34)$. Statistical analyses revealed that changes in swallowing tasks did not influence respiratory phase patterning $(p=0.074)$ but did influence respiratory pause duration $(p<0.0005)$. Furthermore, there was a higher likelihood of penetration-aspiration for swallows that did not exhibit the exhale-swallow-exhale pattern $(p<0.0005)$ and also for swallows with longer respiratory pauses compared to shorter respiratory pauses $(p<0.0005)$.

Conclusions (Including Clinical Relevance): Suboptimal RSC is common in PD and is affected, in part, by variation in swallowing tasks. Swallows with longer respiratory pauses were associated with a greater likelihood of penetration-aspiration. Additionally, swallows with suboptimal respiratory phase patterning were 1.71 times more likely to result in penetration-aspiration than those with optimal patterning. Future research is needed to examine the influence of respiratory-swallow training on swallowing safety in PD.

Relevant Financial Relationships: James Curtis: Has affiliations to disclose;Teachers College, Columbia University:Salary/Stipend:Employment;American Brain Foundation:Grant:Independent contractor (Including contracted research);Parkinson's Foundation:Grant:Independent contractor (Including contracted research);American Academy of Neurology:Grant:Independent contractor (Including contracted research) | Brianna Kiefer: Has affiliations to disclose;NIH:Grant:Independent contractor (Including contracted research) I Michelle Troche: Has affiliations to disclose;Teachers 
College, Columbia University:Salary/Stipend:Employment;Medbridge, Inc.:Royalty:Other Activities;Dysphagia Research Society:Honoraria:Other Activities;Michael J Fox Foundation:Grant:Independent contractor (Including contracted research);National Ataxia Foundation:Grant:Independent contractor (Including contracted research).

Relevant Non-financial Relationships: James Curtis: Has a NonFinancial Disclosure Affliation;Dysphagia Research Society:Professional:Membership;Dysphagia Research Society:Professional: Other volunteer activities I Brianna Kiefer: Has a Non-Financial Disclosure Affliation;Dysphagia Research Society:Professional:Membership | Michelle Troche: Has a Non-Financial Disclosure Affliation;Dysphagia Research Society:Professional:Membership.

\section{Swallow Outcomes Post Mandibular Lingual Release Approach for Oral/Oropharyngeal Cancer: A Retrospective Cohort Study}

\section{Nicola Hardingham ${ }^{1,2}$, Elizabeth Ward ${ }^{1,3}$, Nicola Clayton ${ }^{1,4,5}$, Richard Gallagher $^{6}$}

Purpose: To investigate the acute dysphagia recovery and outcomes in patients following surgical resection of oral and/or oropharyngeal squamous cell carcinoma (OC/OPSCC) using a mandibular lingual release approach (MLRA)

Method(s): A retrospective cohort study was conducted at a tertiary Head and Neck Cancer Centre of patients who underwent surgical resection of OC/OPSCC via MLRA between 2012 and 2017. Data collection included acute medical care, enteral feeding, and swallowing outcomes derived from clinical swallow examination (CSE) and videofluoroscopic swallowing study (VFSS) assessments at baseline, postoperative, and at discharge.

Result(s): In total, 28 patients were eligible for participation (23 males; mean age 63 years). Staging was predominantly T2 (43\%) with surgical closure via free flap in 19 cases. Postoperative speech pathology CSE occurred on average day 5, no significant ( $p=0.231)$ difference between the flap and primary closure groups. Findings revealed $68 \%$ incidence of dysphagia (Functional Oral Intake score FOIS $\leq 6$ ), of which 3 cases had severe dysphagia, 3 with moderate, and 13 had mild symptoms. Following surgery, the majority $(75 \%)$ experienced severe dysphagia (FOIS $\leq 4)-15$ NBM. FOIS scores decreased (baseline median $=6$ to post-op median $=1(\mathrm{NBM})$, representing a statistically significant decline $(p=0.000)$. Twelve patients had VFSS, with silent aspiration observed in 9. Subgroup analysis revealed statistically significant $(p=0.020)$ worse FOIS scores for the flap cohort (median $=1$ ) compared to primary closure group (median $=4$ ), with severe dysphagia more frequent in the flap cohort ( $n=11$ versus $n=1$ in primary closure). At discharge, $43 \%$ had persistent severe dysphagia (FOIS $\leq 4)-7$ NBM. Eleven $(39 \%)$ were managing modified food/fluid (FOIS $=5$ ), and $5(18 \%)$ had mild dysphagia (FOIS $\geq 6$ ). Overall pattern of improvement was statistically significant $(p=0.001)$ between post-op FOIS and discharge. Length of stay ranged substantially within the total cohort (range $4-82$, median $=24$ days $)$ with statistically longer LOS $(p=0.001)$ observed between the flap cohort $($ median $=30)$ and primary closure group $($ median $=12$ )

Conclusions (Including Clinical Relevance): Early post-MLRA dysphagia is common and often severe in presentation. Patients require extended hospital admission with prolonged enteral feeding which may persist post discharge. This cohort requires early intervention by speech-language pathology services to aid swallow rehabilitation.
Relevant Financial Relationships: Nicola Hardingham: Nothing to Disclose I Elizabeth Ward: Nothing to Disclose I Nicola Clayton: Nothing to Disclose I Richard Gallagher: Nothing to Disclose.

Relevant Non-financial Relationships: Nicola Hardingham: Nothing to Disclose I Elizabeth Ward: Has a Non-Financial Disclosure Affliation;DRS:Professional:Membership | Nicola Clayton: Has a Non-Financial Disclosure Affliation;DRS:Professional:Membership | Richard Gallagher: Nothing to Disclose.

\section{Behavioral Interventions Targeting Upper Esophageal Sphincter Opening: A Scoping Review and Appraisal of the Current Evidence Base}

\author{
Sebastian Doeltgen ${ }^{1}$, Harsharan Kaur ${ }^{1}$, Stephanie K. Daniels ${ }^{2}$, \\ Joanne Murray
}

Purpose: Impaired upper esophageal sphincter (UES) opening is a key biomechanical feature often observed in dysphagia, which inhibits bolus transfer into the esophagus. The purpose of this scoping review was to systematically explore behavioural interventions that aim to improve UES opening and to appraise the existing evidence base.

Method(s): A scoping review of six relevant electronic databases (MEDLINE, CINAHL, Ovid Emcare, Web of Science, SCOPUS and ProQuest) was conducted. Studies of behavioural UES interventions published in English that reported on UES-specific outcome measures were included. Interventions and study outcomes were qualitatively characterised into key categories relating to reported intervention type, efficacy and study quality.

Result(s): Of 357 studies initially identified, 18 were included following title, abstract and full text screening. Intervention types aligned with categories of (1) floor of mouth exercises, which were further sub-categorised into the (1a) Shaker exercise and (1b) other strengthening exercises, (2) Mendelsohn manoeuvre, (3) modified balloon dilation, (4) lingual exercises and (5) mixed exercises. Across these interventions, outcomes mainly focussed on UES opening diameter (15/18) and UES opening duration (6/18), reporting variable success. Aspiration was evaluated in 10/18 studies and improved in $4 / 18$. The included evidence base was limited by issues with reporting on methodology employed, selection of participants (only $7 / 18$ studies evaluated individuals with demonstrated UES impairment) and assessor blinding.

Conclusions (Including Clinical Relevance): Several behavioural interventions targeting UES opening provide promising evidence for improved UES opening and duration. However, while proof of concept studies in healthy controls provide some support, the evidence base for patients with demonstrated UES opening impairment is still relatively small and limited by study quality. Future high-quality research in individuals with demonstrated UES impairment is needed to strengthen the depth and quality of the evidence base.

Relevant Financial Relationships: Sebastian Doeltgen: Has affiliations to disclose;Flinders University:Salary/Stipend:Employment I Harsharan Kaur: Nothing to Disclose I Stephanie Daniels: Has affiliations to disclose;University of Houston, TX:Salary/ Stipend:Employment । Joanne Murray: Has affiliations to disclose;Flinders University:Salary/Stipend:Employment.

Relevant Non-financial Relationships: Sebastian Doeltgen: Has a Non-Financial Disclosure Affliation;Dysphagia Research Society:Professional:Membership I Harsharan Kaur: Nothing to Disclose I Stephanie Daniels: Has a Non-Financial Disclosure Affliation;Dysphagia Research Society:Professional:Membership I Joanne Murray: Nothing to Disclose. 


\section{Comparison of Patient Reported and Caregiver Reported Swallowing-Related Quality-of-Life in Parkinson's Disease}

\section{Allison S. Zimmerman ${ }^{1}$, Kendrea L. Garand ${ }^{1}$, Julie Estis ${ }^{1}$, Samantha Shune $^{2}$, Kimberly Smith ${ }^{1}$}

Purpose: To identify if differences existed between patient- and caregiver-reported swallowing-related quality-of-life in patients with Parkinson's disease (PD).

To identify potential factors influencing differences between patient and caregiver scores.

Method(s): Participants included both patients diagnosed with PD and their identified primary caregivers. Eligible participants were electronically administered demographic and swallowing-related quality-of-life (SWAL-QOL) questionnaires. A Wilcoxon Signed-Rank test was completed to compare SWAL-QOL score means between patients and caregivers. Factors potentially influencing scores were explored using Spearman coefficient correlation tests. The HolmBonferroni method was applied to adjust for multiple comparisons.

Result(s): 36 patient-caregiver pairs were available for analysis. The mean SWAL-QOL score reported by patients and caregivers was $78.47( \pm 15.5)$ and $78.53( \pm 13.9)$. There was a moderate degree of reliability and agreement between the patient score and the caregiver score, with the average ICC measures being 0.598 (95\% CI [0.358, $0.748])(F(71,72)=2.451, p<0.0001)$

Eight variables were tested to assess influence on SWAL-QOL scores: age, employment status, sex, ethnicity and race, previous history of swallowing evaluation or treatment, caregiver concern about patient cognition, and time since the onset of disease. After adjusting for multiple comparisons, only caregiver burden remained statistically significant $(p=0.001)$. When caregivers reported caregiver burden, they were more likely to report lower quality-of-life scores for the individual with PD.

Conclusions (Including Clinical Relevance): No significant differences were observed between patient-reported and caregiver-reported SWAL-QOL scores. The lack of difference in SWAL-QOL scores suggest support for the use of proxy SWAL-QOL scores in PD. Of clinical importance, caregiver burden was found to be a statistically significant influential factor on caregiver ratings of swallowing-related quality of life. Caregiver burden may contribute to poorer dyadic management and/or illness appraisal resulting in lower SWAL-QOL scores on behalf of the caregiver. The Theory of Dyadic Illness Management suggests that caregivers with low burden are likely better equipped to identify and manage patient's needs. Therefore, managing caregiver burden appropriately may contribute to earlier identification and better management of dysphagia.

Relevant Financial Relationships: Allison Zimmerman: Nothing to Disclose I Kendrea Garand: Nothing to Disclose I Julie Estis: Nothing to Disclose I Samantha Shune: Nothing to Disclose I Kimberly Smith: Nothing to Disclose.

Relevant Non-financial Relationships: Allison Zimmerman: Nothing to Disclose I Kendrea Garand: Nothing to Disclose I Julie Estis: Nothing to Disclose I Samantha Shune: Nothing to Disclose I Kimberly Smith: Nothing to Disclose.

\section{Algorithmic Hyoid Tracking and C2-C4 Length Measurement on Artificial Intelligence Predicted Segmentations}

\author{
Nadeem M. Shaheen ${ }^{1}$, Rodolfo Peña-Chávez ${ }^{2}$, Chris \\ Ulmschneider $^{3}$, Ryan Burdick ${ }^{2,4}$, Joanne Yee ${ }^{5,4}$, Atsuko Kurosu ${ }^{3}$, \\ Bryan Bednarz ${ }^{1}$, Nicole Rogus-Pulia ${ }^{2,4,5}$
}

Purpose: Videofluoroscopic swallow (VFS) studies generally require video manipulation between software and manual input to measure anatomic regions of interest. Hyoid bone displacement as well as pharyngeal residue estimation (which requires the cervical vertebrae 2 (C2) to 4 (C4) length for size normalization) are two such measures. Algorithmic determination of these measures from Artificial Intelligence (AI)-based segmentations could allow for routine use without manual input to support clinical decision-making or image analysis for research. This study aimed to create and test a hyoid tracking algorithm as well as $\mathrm{C} 2$ to $\mathrm{C} 4$ length measurement using AI-based segmentations.

Method(s): Eleven VFS videos of thin-liquid swallows recorded at 30 frames per second were selected from a cohort of patients with post-stroke dysphagia. The hyoid, C2, and C4 were manually segmented on the first subswallow of each bolus by a trained rater. A neural network was trained to produce accurate anatomical segmentations. The hyoid position was tracked using the center of mass of each segment on a frame by frame basis. Hyoid position was normalized to its origin to be comparable to the manual tracking method. The $\mathrm{C} 2$ to $\mathrm{C} 4$ length (distance from the anterior inferior portion of $\mathrm{C} 2$ to the anterior inferior portion of $\mathrm{C} 4$ ) was determined on the rest frame using a custom algorithm.

Result(s): When comparing the algorithmic $\mathrm{C} 2$ to $\mathrm{C} 4$ length measure to manual determination, an intraclass correlation coefficient (ICC) of 0.98 was observed. For nine $\mathrm{C} 2$ to $\mathrm{C} 4$ length measures, the average percent error was $3.2 \%$ with a standard deviation of $1.6 \%$. On average, across all frames of videos, the hyoid tracking error was 16 pixels on a $480 \times 720$-pixel image.

Conclusions (Including Clinical Relevance): The resulting $\mathrm{C} 2$ to $\mathrm{C} 4$ measures on AI predicted segments using a generalized algorithm achieved a high level of consistency compared to the expert rater that exceeded a minimally acceptable ICC $(0.80)$ for manual raters. While hyoid tracking followed the general motion of the hyoid, further action needs to be taken to reduce tracking error. Future work will focus on optimization of these AI predicted measures as well as development of new measures (e.g., pharyngeal residue) that in combination will provide real-time clinically relevant information.

Relevant Financial Relationships: Nadeem Shaheen: Has affiliations to disclose;University of Wisconsin Madison:Salary/ Stipend:Employment I Rodolfo Peña-Chávez: Has affiliations to disclose;University of Wisconsin Madison:Salary/Stipend:Employment I Chris Ulmschneider: Has affiliations to disclose;University of Wisconsin Madison:Salary/Stipend:Employment I Ryan Burdick: Has affiliations to disclose;University of Wisconsin Madison:Salary/ Stipend:Employment I Joanne Yee: Has affiliations to disclose;William S. Middleton Veterans Affairs Hospital:Salary/ Stipend:Employment I Atsuko Kurosu: Has affiliations to disclose;National Institute of Health:Salary/ Stipend:Employment;University of Wisconsin Madison:Salary/ Stipend:Employment | Bryan Bednarz: Has affiliations to disclose;University of Wisconsin Madison:Salary/Stipend:Employment I Nicole Rogus-Pulia: Has affiliations to disclose;University of Wisconsin Madison:Salary/Stipend:Employment;National Institute of Health:Grant:Independent contractor (Including contracted research);William S. Middleton Memorial Veterans Hospital:Salary/ Stipend:Employment;University of Wisconsin Medical Foundation:Salary/Stipend:Employment.

Relevant Non-financial Relationships: Nadeem Shaheen: Nothing to Disclose I Rodolfo Peña-Chávez: Has a Non-Financial Disclosure Affliation;Dysphagia Research Society:Professional:Membership । Chris Ulmschneider: Nothing to Disclose I Ryan Burdick: Nothing to Disclose | Joanne Yee: Has a Non-Financial Disclosure Affliation;Dysphagia Research Society:Professional:Membership | Atsuko Kurosu: Has a Non-Financial Disclosure Affliation;Dysphagia Research Society:Professional:Membership | Bryan Bednarz: Nothing to Disclose I Nicole Rogus-Pulia: Has a Non-Financial Disclosure 
Affliation;Dysphagia Research Society:Professional:Board membership;Dysphagia Research Society:Professional:Membership.

\section{A Preliminary Videofluoroscopic Investigation of Swallowing Physiology and Function in People Living with Severe Dementia}

\section{Ashwini M. Namasivayam-MacDonald ${ }^{1}$, Ashlee Ruff ${ }^{2}$, Luis Riquelme ${ }^{2,3}$}

Purpose: Dysphagia is a common symptom experienced by people living with dementia (PwD), particularly for those with advanced disease progression. However, we lack understanding of the discrete changes in swallowing physiology seen in this subset of the population, leading to questions surrounding optimal management approaches during late stages of the disease. This prospective study sought to describe the pathophysiology of dysphagia in PwD presenting with severe cognitive impairment using videofluoroscopy swallowing studies (VFs).

Method(s): Data were collected from 9 patients (4 male; 81-97 years) with a medical diagnosis of dementia admitted to acute care requiring a swallowing assessment. Cognition was assessed using the Montreal Cognitive Assessment and all scores were $<11$, indicating severe cognitive impairment. VFs were performed on all participants; only natural sips of thin liquid were extracted for this study. Three blinded raters scored VF clips for safety [Penetrationaspiration Scale (PAS)], efficiency [Normalized Residue Ratio Scale (NRRS)], timing [Pharyngeal Transit Time, Swallow Reaction Time, Laryngeal Vestibule Closure Reaction Time, Upper Esophageal Sphincter Opening Duration], and kinematics [Maximum Pharyngeal constriction]. Impairment thresholds from existing literature were defined to characterize swallowing physiology and function. Fisher's Exact tests and Pearson's correlations were used to conduct a preliminary exploration of associations between swallowing physiology and function.

Result(s): Unsafe swallowing (PAS $>2$ ) was seen in 8/9 PwD and clinically significant residue (NRRS scores $>0.004$ vallecular; $>0.018$ pyriform) was seen in 9/9 PwD. Compared to published norms, we identified significant differences in residue, swallow reaction time, laryngeal vestibule closure reaction time, and UES opening duration. There were no associations identified between postswallow residue and pharyngeal constriction, nor between penetration-aspiration and laryngeal vestibule closure reaction time or swallow reaction time.

Conclusions (Including Clinical Relevance): While there are clear signs of swallowing impairment (airway invasion, significant residue) in people living with severe dementia the mechanisms leading to these impairments are unclear. Further work is needed to explore a greater range of food and liquid textures and to identify additional physiological mechanisms underlying dysphagia in this population.

Relevant Financial Relationships: Ashwini NamasivayamMacDonald: Has affiliations to disclose;McMaster University:Salary/ Stipend:Employment I Ashlee Ruff: Has affiliations to disclose;Barrique Speech-Language Pathology:Salary/Stipend:Employment I Luis Riquelme: Has affiliations to disclose;Barrique Speech-Language Pathology:Salary/Stipend:Ownership;New York Medical College:Salary/Stipend:Employment.

Relevant Non-financial Relationships: Ashwini NamasivayamMacDonald: Has a Non-Financial Disclosure Affliation;Dysphagia Research Society:Professional:Membership;Dysphagia Research Society:Professional:Volunteer membership on advisory committee or review panels;American Speech-Language-Hearing Association:Professional:Membership;American Speech-Language-Hearing
Association:Professional:Volunteer membership on advisory committee or review panels I Ashlee Ruff: Nothing to Disclose I Luis Riquelme: Has a Non-Financial Disclosure Affliation;Dysphagia Research Society:Professional:Membership;IDDSI:Professional:Board membership;American Speech-Language-Hearing Association:Professional:Membership;American Speech-LanguageHearing Association:Professional:Volunteer membership on advisory committee or review panels.

\section{Evaluation of Three-Dimensional Hyoid Bone Movements Using 320-ADCT in Patients After Hemiglossectomy and neck Dissection}

\author{
Junko Nakajima ${ }^{1}$, Hirokazu Saito ${ }^{2}$, Yukari Ishii ${ }^{3}$, Satoru Ogane ${ }^{4}$, \\ Katsuhiko Sakai $^{1}$, Takeshi Nomura ${ }^{2}$, Nobuyuki Matsuura ${ }^{1}$
}

Purpose: Surgical treatments for head and neck cancer can cause swallowing complications. For glossectomy patients, the resection of hyoid muscles and asymmetrical-reconstructed tongue affect hyoid bone mobility. For the analysis of hyoid bone movements, VFSS targeting the anterior point of the hyoid bone in the lateral image has been used in many studies. However, the results of such analysis may not represent post-surgical hyoid bone movements because the hyoid muscles are asymmetrical in these patients.

This study aimed to clarify three-dimensional hyoid bone movements during swallowing in patients who underwent hemiglossectomy and unilateral neck dissection using 320-ADCT scans.

Method(s): This study included 7 patients who underwent hemiglossectomy and unilateral neck dissection. We evaluated the swallowing function using 320-ADCT 1 week before and 1 month after the surgery. A bolus of $4 \mathrm{ml}$ of barium sulfate solution was administered to each patient, and we analyzed the following parameters: (1) the position of the hyoid bone at rest; (2) the highest position of the hyoid bone during swallowing, at 3 points on the hyoid bone: most anterior point of the middle body and, right and left greater horn. Result(s): After surgery, the hyoid bone at rest was displaced toward the non-resected side. During the swallowing, the distance traversed decreased significantly after the surgery at every analyzed point on the hyoid bone. The affected side moved significantly lesser compared to the non-affected side (affected side: $11.1 \pm 5.89 \mathrm{~mm}$, nonaffected side: $16.4 \pm 7.1 \mathrm{~mm}$ ); however, it was not significant before surgery (affected side: $21.3 \pm 7.0 \mathrm{~mm}$, non-affected side: $22.3 \pm 7.0 \mathrm{~mm}$ ). The average distance traversed at the non-affected greater horn in the upward direction was relatively well maintained even after surgery compared to other points (middle point:10.3 mm, non-affected side: $16 \mathrm{~mm}$, affected side: $9.6 \mathrm{~mm}$ ).

Conclusions (Including Clinical Relevance): This study is the first study to clarify the asymmetrical hyoid bone movement during swallowing after hemiglossectomy and neck dissection. The swallowing evaluation using 320-ADCT could detect the rotation on the axial plane during swallowing. Surgical invasion decreased hyoid bone movement; however, it seems that the non-affected side works sufficiently to compensate for the reduced functions.

Relevant Financial Relationships: Junko Nakajima: Nothing to Disclose I Hirokazu Saito: Nothing to Disclose I Yukari Ishii: Nothing to Disclose I Satoru Ogane: Nothing to Disclose I Katsuhiko Sakai: Nothing to Disclose | Takeshi Nomura: Nothing to Disclose | Nobuyuki Matsuura: Nothing to Disclose.

Relevant Non-financial Relationships: Junko Nakajima: Nothing to Disclose I Hirokazu Saito: Nothing to Disclose I Yukari Ishii: Nothing to Disclose I Satoru Ogane: Nothing to Disclose I Katsuhiko Sakai: Nothing to Disclose | Takeshi Nomura: Nothing to Disclose I Nobuyuki Matsuura: Nothing to Disclose. 


\section{Scoring the Penetration-Aspiration Scale (PAS) in Two Conditions: A Reliability Study}

\author{
Munirah Alkhuwaiter ${ }^{1}$; Kate Davidson ${ }^{3}$; Theresa Hopkins- \\ Rossabi $^{1}$; Bonnie Martin-Harris ${ }^{1,2}$
}

Purpose: The Penetration-aspiration scale (PAS) is a widely applied metric for identifying the presence and severity of airway invasion during swallowing, yet variability exists in the scoring conditions used by raters across clinical laboratories. The impact of scoring conditions on PAS reliability has not been investigated and may impact conclusions drawn from study results. This project examined differences in PAS rater reliability and score accuracy between two scoring conditions in student and clinician raters.

Method(s): Seven students and 4 experienced ( $M=8$ yrs) SLP clinicians participated as raters. Raters viewed a videofluoroscopic recording demonstrating definitions and applied PAS scoring. Two clinical researcher SLPs served as "gold standard" raters.15 MBSS records were selected and represented the full range of PAS scores. The 15 MBSS (126 swallow tasks) were trimmed and randomized for rater distribution. To assess inter-rater reliability, raters were divided into: Contextual scoring $(N=6)$ when raters scored each swallow task within the context of a MBSS of the same patient, and Randomized $(\mathrm{N}=5)$ when raters scored single swallow tasks from multiple patient MBSS and blinded to surrounding swallows. Raters switched scoring condition after a two-week break. For intra-rater reliability, raters rescored $25 \%$ of swallow tasks from each condition. Score accuracy was measured by comparing rater scores with consensus scores between the gold standard raters.

Result(s): Student inter-, intra-rater reliability and accuracy were higher in the Randomized condition (ICC $=0.67,0.76,0.74$ ) compared to the Contextual $(\mathrm{ICC}=0.55,0.73,0.69)$. Clinician inter-rater reliability were equivalent in the two conditions $(\mathrm{ICC}=70)$. Intrarater reliability was higher in the Randomized $(\mathrm{ICC}=0.86$ ) compared to the Contextual (ICC $=0.80$ ). Accuracy was higher in the Contextual (ICC $=0.78$ ) compared to the Randomized (ICC $=0.76$ ). None of the differences between conditions were statistically significant based on $95 \%$ confidence intervals.

Conclusions (Including Clinical Relevance): In this preliminary investigation, rater reliability and score accuracy were comparable between the contextual and randomized conditions. If findings are reproduced in larger samples, splicing and randomizing MBSSs may reduce laboratory efficiency without significant improvement in PAS reliability and accuracy.

Relevant Financial Relationships: Munirah Alkhuwaiter: Nothing to Disclose I Kate Davidson: Has affiliations to disclose;NIH/NIDCD:Grant:Other Activities I Theresa Hopkins-Rossabi: Nothing to Disclose I Bonnie Martin-Harris: Has affiliations to disclose;NIH/NIDCD: Grant:Other Activities;Northwestern University:Salary/Stipend: Employment;Veteran's Administration:Salary/Stipend:Employment.

Relevant Non-financial Relationships: Munirah Alkhuwaiter: Nothing to Disclose I Kate Davidson: Nothing to Disclose I Theresa Hopkins-Rossabi: Nothing to Disclose I Bonnie Martin-Harris: Has a Non-Financial Disclosure Affliation;Dysphagia Research Society:Professional:Membership.

\section{Neurodevelopmental and Feeding Routes at 24 Months in Children with Oropharyngeal Dysphagia}

\section{Deborah S. Levy ${ }^{1}$}

Purpose: To analyze and describe clinical diagnosis of neurodevelopmental and feeding routes in children at 24 months of age diagnosed with oropharyngeal dysphagia.
Method(s): Retrospective review performed using the medical records of the patients that were the children underwent Videofluoroscopic Swallowing Study (VFSS) and were followed up at a hospital Outpatient Clinic for Pediatric Dysphagia. Those children whose medical records were incomplete were excluded. The variables were described using median and interquartile range Md (IIQ25IIQ75) for continuous variables, and absolute and relative frequencies $N(\%)$ for categorical variables. The non-parametric statistical tests used to compare the medians between groups were Mann-Whitney $U$ and Kruskal-Wallis.

Result(s): The final study group was composed of 55 children, with median age of $8(4-15)$ months. There was a predominance of females $(30,54.5 \%)$ and children under the age of 12 months $(36,65.5 \%)$ at the time of VFSS. The results showed penetration $(16,29.09 \%)$ or aspiration $(14,25.45 \%)$, silent aspiration in the majority of cases (12, $85.71 \%)$. At 24 months, $42(76.36 \%)$ children were classified as ageappropriate and $13(23.63 \%)$ as delay for their neurodevelopment according to clinical diagnosis of pediatrician or pediatric neurologist. The oral nutrition exclusively was predominantly, observed in 34 $(61.81 \%)$ children, while $10(18.18 \%)$ received exclusive enteral nutrition and $11(20 \%)$ oral and enteral concomitantly. Among children with enteral nutrition, $15(71.42 \%)$ were gastrostomized. Older children at the time of the VFSS, 10.5 (5-15.25) months, were receiving exclusive enteral nutrition at 24 months, regarding those children with oral and enteral, 4 (2-6.50) months, and those with exclusive oral nutrition, $6(2-11.50)$ months. The difference was $p=0.034$. Older children at the time of the VFSS, $7.5(4.25-12)$ months, were diagnosed with neurodevelopmental disorders at 24 months, compared to the younger ones, $4(2-5)$ months, in whom prevailed the normal diagnosis. The difference was $p=0.014$.

Conclusions (Including Clinical Relevance): Late diagnosis by VFSS was related to exclusive enteral nutrition and neurodevelopment disorders at 24 months. For children with neurodevelopment disorders and dysphagia, growth may not improve swallowing biomechanics as expected. The data suggest a severe and adverse diagnosis for effective oral feeding, often with the adoption of gastrostomy.

Relevant Financial Relationships: Deborah Levy: Nothing to Disclose.

Relevant Non-financial Relationships: Deborah Levy: Nothing to Disclose.

\section{Determining the Validity of the Tongueometer in Comparison to the Iowa Oral Performance Instrument}

Nazia Mohsin ${ }^{1}$, Rebekah Guastella ${ }^{3}$, Stefania Oppedisano $^{3}$, Ashwini M. Namasivayam-MacDonald ${ }^{2}$

Purpose: Measuring tongue strength supports the objective assessment and treatment of oral motor impairments, such as dysphagia and dysarthria. As new tongue pressure manometers come to market it is important that they are validated against the current gold standard prior to being implemented in research and clinical practice. This study investigates the concurrent validity of a new, relatively inexpensive device to measure tongue strength, the Tongueometer, in comparison to the current gold standard tongue pressure manometer (the Iowa Oral Performance Instrument $\left(\mathrm{IOPI}^{\circledR}\right)$ ).

Method(s): Three different tongue pressure measurements were conducted on each of the 45 participants ( 34 female, $M=46.9$ years) using the two devices: (1) maximum anterior pressure; (2) maximum posterior pressure; and (3) regular effort saliva swallows. The maximum value across three trials was calculated for each task for each participant. Concurrent validity was assessed by determining the 
Pearson's correlation coefficient for the relationship between the two devices for each of the three tasks. Paired $t$ tests were also used to determine if the measures taken by the two devices were similar.

Result(s): There were significant $(p<0.001)$, strong, positive correlations $(r=0.73-0.89)$ between the two devices across all tasks, confirming concurrent validity. However, $t$ test results revealed that there were no significant differences in anterior tongue pressure measures, but there were significant differences in posterior tongue and swallowing pressure measures $(\mathrm{p}<0.05)$. Both instruments demonstrated similar variability in the tongue pressure measures across trials for each task.

Conclusions (Including Clinical Relevance): Overall, the Tongueometer is a low-cost tool that demonstrates strong concurrent validity across anterior pressure, posterior pressure, and swallowing tasks when compared to the IOPI. The two devices took very similar anterior tongue pressure measurements but measured posterior and swallow pressures differently. This could be due to differences in bulb shape, material, and volume. Further analyses of tongue pressure measures between the devices by age group with an increased sample size may shed light on why the devices take certain measures differently.

Relevant Financial Relationships: Nazia Mohsin: Has affiliations to disclose;McMaster University-Aging Swallow Research Lab:Salary/Stipend:Employment I Rebekah Guastella: Has affiliations to disclose;McMaster University-Aging Swallow Research Lab:Salary/Stipend:Employment I Stefania Oppedisano: Has affiliations to disclose;McMaster University-Aging Swallow Research Lab:Salary/Stipend:Employment I Ashwini Namasivayam-MacDonald: Has affiliations to disclose;McMaster University:Salary/ Stipend:Employment.

Relevant Non-financial Relationships: Nazia Mohsin: Nothing to Disclose | Rebekah Guastella: Nothing to Disclose | Stefania Oppedisano: Nothing to Disclose I Ashwini Namasivayam-MacDonald: Has a Non-Financial Disclosure Affliation;Dysphagia Research Society:Professional:Membership;American Speech-Language-Hearing Association:Professional:Membership;Dysphagia Research Society:Professional:Volunteer membership on advisory committee or review panels;E2 Scientific_-Tongueometer:Professional: Other volunteer activities.

\section{Are Hyoid Bone Kinematics Associated with Swallowing Safety?}

\section{Sana Smaoui ${ }^{1,2}$, Melanie Peladeau-Pigeon ${ }^{2}$; Catriona M. Steele ${ }^{2,1}$}

Purpose: Hyoid bone movement is commonly evaluated in videofluoroscopic swallowing studies (VFSS), yet research remains equivocal regarding links between hyoid movement and swallowing safety. Our goal was to determine the association between hyoid kinematics, swallowing safety as measured by the penetration-aspiration Scale (PAS), and parameters measuring laryngeal-vestibuleclosure (LVC) timing and integrity.

Method(s): This study involved secondary analysis of an existing dataset from an industry-funded study of 305 participants (152 males) with a mean age of 72 (range 28-100), who were considered at-risk for non-congenital, nonsurgical, and non-oncological oropharyngeal dysphagia. Participants completed a VFSS, including 6 boluses of thin-liquid barium $(20 \% \mathrm{w} / \mathrm{v})$. Blinded duplicate ratings of swallowing safety, timing and kinematics were completed using the ASPEKT method. Swallowing parameters were categorized as typical (i.e. within the healthy interquartile range) or atypical $(<$ the 25 th\%ile or $>$ the 75 th\%ile) based on recently published reference values (Steele et al. 2019; Smaoui et al. 2020). Cross-tabulations, Chi Square tests and odds ratios were computed to determine the associations between atypical values for hyoid burst duration, hyoid $X Y$ peak position, and hyoid $X Y$ speed, PAS, time-to-LVC and LVC integrity. Result(s): The odds of an unsafe swallow, defined as PAS $\geq 3$, did not differ significantly between swallows with typical versus prolonged hyoid burst duration. However, strong associations were found between swallowing safety, hyoid $X Y$ peak position and hyoid $X Y$ speed. Reduced hyoid $X Y$ peak position $(<$ the 25 th \%ile reference threshold) was associated with significantly increased odds of PAS $\geq 3$ (2.2-fold), incomplete LVC (3.8-fold) and prolonged time-toLVC (1.5-fold). Similarly, reduced hyoid $X Y$ speed was associated increased odds of PAS $\geq 3$ (1.92-fold), incomplete LVC (2.9-fold) and prolonged time-to-LVC (1.5-fold). The combination of reduced hyoid $X Y$ peak position and speed with incomplete $\mathrm{LVC}$ and atypical prolonged time-to-LVC, increased the odds of PAS $\geq 3$ by 10.9-fold. Conclusions (Including Clinical Relevance): These results corroborate previous studies suggesting links between hyoid movement and swallowing safety. In particular, reductions in hyoid XY peak position and speed were highly predictive of PAS $\geq 3$ in this heterogeneous sample of adults with risk for oropharyngeal dysphagia.

Relevant Financial Relationships: Sana Smaoui: Has affiliations to disclose;KITE-Toronto Rehabilitation Institute, University Health Network:Salary/Stipend:Employment I Melanie Peladeau-Pigeon: Has affiliations to disclose;KITE-Toronto Rehabilitation Institute, University Health Network:Salary/Stipend:Employment I Catriona Steele: Has affiliations to disclose;Nestle Health Science:Grant:Independent contractor (Including contracted research);KITE-Toronto Rehabilitation Institute, University Health Network:Salary/ Stipend:Employment;National Institute of Deafness and Other Communication Disorders:Grant:Independent contractor (Including contracted research);Nestle Health Science:Speaking fee:Teaching and speaking.

Relevant Non-financial Relationships: Sana Smaoui: Nothing to Disclose I Melanie Peladeau-Pigeon: Nothing to Disclose I Catriona Steele: Has a Non-Financial Disclosure Affliation;International Dysphagia Diet Standardisation Initiative:Professional:Board membership.

\section{Psychometric Properties of Patient-Reported Outcome Measures for Dysphagia in Head and Neck Cancer: A Scoping Review}

Beatrice Manduchi ${ }^{1,2,3}$, Zhiyao Che ${ }^{1,2,3}$, Margaret I. Fitch ${ }^{4}$, Jolie Ringash $^{5}$, Doris Howell ${ }^{4,6}$, Rosemary Martino ${ }^{1,2,3,7,8}$

Purpose: Dysphagia is one of the main complaints for patients with head and neck cancer (HNC). Dysphagia-related patient-reported outcome measures (PROMs) are critical to provide patient information about the impact of disease and treatment, tailor interventions, and improve communication between patients and healthcare providers. Presently, there is no inventory specific to PROMs for dysphagia in $\mathrm{HNC}$ and their psychometric properties. We performed a scoping review to identify PROMs and their psychometric properties, specific to dysphagia symptoms, functional status, swallowing-related health, and quality of life (QoL) in HNC.

Method(s): We searched 5 electronic databases from inception to May 2019 for all primary studies in any language and design detailing PROM development, psychometric testing, interpretability, and crosscultural adaptation. Eligibility criteria targeted PROMs for patients with $\mathrm{HNC}$ ( $\geq 90 \%$ of the study sample) with $\geq 20 \%$ of their items pertaining to swallowing. Two independent raters screened all citations and a third rater resolved discrepancies. Accepted full-text articles were screened for eligibility by one rater. PROMs and their psychometric properties were summarized descriptively according to the COSMIN taxonomy. 
Result(s): Of the 8838 unique citations retrieved, 1161 moved to fulltext screening and 83 met the inclusion criteria. The final yield consisted of 20 PROMs focused on the following dysphagia domains: symptoms $(n=6)$, functional status $(n=7)$, health status $(n=2)$, and QoL $(n=5)$. Across all PROMs, nine were specific to swallowing, and five were translated into multiple languages. All PROMs were tested for internal consistency, test-retest reliability, and construct validity, but only select PROMs reported: content $(n=5)$, face $(n=2)$, and criterion $(n=9)$ validity; feasibility $(n=5)$; responsiveness $(n=5)$; and minimal important difference $(n=5)$.

Conclusions (Including Clinical Relevance): This study identified 20 PROMs targeting dysphagia in HNC. Of these, all included psychometric testing for reliability and construct validity, but only few assessed for other properties related to change. Future testing is required to ascertain whether existing tools can accurately detect longitudinal changes and clinically significant differences, both of which are measures essential for clinical and research purposes.

Relevant Financial Relationships: Beatrice Manduchi: Nothing to Disclose I Zhiyao Che: Nothing to Disclose I Margaret Fitch: Nothing to Disclose I Jolie Ringash: Nothing to Disclose I Doris Howell: Nothing to Disclose I Rosemary Martino: Nothing to Disclose.

Relevant Non-financial Relationships: Beatrice Manduchi: Nothing to Disclose I Zhiyao Che: Nothing to Disclose I Margaret Fitch: Nothing to Disclose I Jolie Ringash: Nothing to Disclose | Doris Howell: Nothing to Disclose I Rosemary Martino: Has a Non-Financial Disclosure Affliation;DRS membership:Professional:Membership.

\section{The Impact of Automated Milk Delivery on Infant Feeding Performance and Kinematics}

\section{Kendall E. Steer ${ }^{1}$, Chloe E. Edmonds ${ }^{2}$, Francois D. Gould ${ }^{5}$, Khaled Adjerid $^{3}$, Laura E. Bond ${ }^{1}$, Rebecca Z. German', Christopher J. Mayerl ${ }^{4}$}

Purpose: Infant feeding is a complex sensorimotor process that involves the acquisition, transport, and swallowing of food using multiple structures. Disruptions in performance in any of these activities are potentially detrimental for swallow physiology. Infants actively acquire food by depressing the mandible and tongue to draw milk from the breast into the oral cavity and then transport food posteriorly to the valleculae by moving the tongue in a wave. Swallowing is then triggered by sensory stimulation in the valleculae. We used a validated animal model (infant pigs) to test whether the automated delivery of milk into the oral cavity would impact infant feeding performance, with the hypothesis that automated milk delivery would alter sucking physiology and also have downstream impacts on swallow performance.

Method(s): We surgically implanted radiopaque markers into three points on the tongue midline, as well as on the mental process, hyoid, and soft palate. We filmed infant pigs using biplanar videofluoroscopy (100 fps) when feeding on a bottle and at two different automated volume delivery rates and tracked. Following data collection, we tracked radiopaque markers in 3D and measured swallow safety using the Infant Mammalian Penetration Aspiration Scale (IMPAS).

Result(s): We found that the anterior tongue moved significantly more when sucking during bottle feeding than during automated milk delivery. Furthermore, during swallowing, the posterior tongue also moved significantly more when bottle feeding than during automated milk delivery. Despite these differences in kinematics, we observed no changes in IMPAS scores.

Conclusions (Including Clinical Relevance): These results suggest that automated milk delivery results in reduced tongue movement, which impacts both the parts of the tongue generating suction (anterior) and swallowing (posterior). By delivering milk using an automated system, the tongue is likely producing less suction, which has downstream impacts on feeding kinematics by altering tongue movements during the swallow. Our work indicates that interventions designed to treat dysphagia in infants should account for the complex sensorimotor integration involved in infant feeding, and that altering the sensory stimulation associated with food acquisition and transport may be a powerful tool to improving feeding outcomes in infants with feeding problems.

Relevant Financial Relationships: Kendall Steer: Nothing to Disclose I Chloe Edmonds: Nothing to Disclose I Francois Gould: Nothing to Disclose I Khaled Adjerid: Nothing to Disclose I Laura Bond: Nothing to Disclose I Rebecca German: Nothing to Disclose I Christopher Mayerl: Nothing to Disclose.

Relevant Non-financial Relationships: Kendall Steer: Nothing to Disclose I Chloe Edmonds: Nothing to Disclose I Francois Gould: Nothing to Disclose I Khaled Adjerid: Nothing to Disclose I Laura Bond: Nothing to Disclose I Rebecca German: Nothing to Disclose I Christopher Mayerl: Nothing to Disclose.

\section{The Trajectory of Head and Neck Lymphedema Following Chemoradiotherapy for Head and Neck Cancer: Prevalence, Improvement Trends and the Association with Dysphagia}

\section{Claire Jeans $^{1,2}$, Bena Brown ${ }^{1,3,4}$, Elizabeth Ward ${ }^{1,3}$, Anne Vertigan $^{5,6,7}$, Amanda Pigott ${ }^{1,3,8}$, Jodie Nixon ${ }^{1,8}$, Chris Wratten', May Boggess ${ }^{10}$}

Purpose: Head and neck lymphedema (HNL) is prevalent following head and neck cancer (HNC) management. Patients with more severe internal HNL (I-HNL) and external HNL (E-HNL) are known to experience dysphagia. However, there are limited data on the pattern of HNL over time and how this relates to dysphagia. A prospective longitudinal cohort study was conducted to examine (a) the trajectory of I-HNL and E-HNL in HNC patients up to 12 months postchemoradiotherapy (CRT), and (b) explore the relationship between HNL and swallowing.

Method(s): Thirty-three patients $(91 \%$ male, $91 \%$ oropharyngeal tumours, and $67 \% \mathrm{~T} 1-2)$ were assessed at 3, 6, and 12 months postCRT. I-HNL was rated with Patterson's Radiotherapy Edema Rating Scale, and E-HNL with the MD Anderson Cancer Centre Lymphoedema Rating Scale. Swallowing was assessed via instrumental, clinical, and patient-reported outcome measures (Penetration-aspiration Scale [PAS], Mann Assessment of Swallowing Ability-Cancer [MASA-C], Functional Oral Intake Scale [FOIS], Vanderbilt Head and Neck Symptom Survey [VHNSS]). Associations between HNL and swallowing were examined with multivariable regression models. Result(s): The majority had moderate/severe I-HNL at 3 months (96\%) and 6 months (84\%), with some reduction to $65 \%$ at 12 months $(p=0.006)$. E-HNL was most prevalent at 3 months (71\%), some improvement at 6 months $(58 \%)$, and in contrast the I-HNL, had largely resolved by 12 months $(10 \%, p<0.001)$. At 6 months, higher severities of I-HNL and E-HNL, and a higher number of internal sites with HNL were associated with worse MASA-C scores ( $p=0.01,<0.001,0.002$, respectively), and worse PAS scores $(p=0.001,0.012,0.011$, respectively). A higher severity of E-HNL and a higher number of internal sites with HNL were also associated with worse FOIS scores ( $p=0.005,0.021$, respectively), and worse swallow solid scores on the VHNSS $(p=0.039,0.015$, respectively).

Conclusions (Including Clinical Relevance): I-HNL and E-HNL were prevalent at 3 months and small improvements were seen at 
6 months. While E-HNL largely resolves in most by 12 months, two thirds continue to have moderate/severe I-HNL. Strong associations were observed between HNL and dysphagia at 6 months, with patients with higher severities of I-HNL and E-HNL and more diffuse I-HNL more likely to have more severe penetration and/or aspiration, increased diet modification, and more difficulty eating solid foods.

Relevant Financial Relationships: Claire Jeans: Has affiliations to disclose;NSW Health:Salary/Stipend:Employment;GenesisCare Newcastle:Consulting fee:Independent contractor (Including contracted research);Hunter ENT:Consulting fee:Independent contractor (Including contracted research);Hunter Cancer Research Alliance:Scholarship:Independent contractor (Including contracted research);James Lawrie Head and Neck Grant Fund, Calvary Mater Newcastle:Grant:Independent contractor (Including contracted research) I Bena Brown: Has affiliations to disclose;QLD Health:Salary/Stipend:Employment I Elizabeth Ward: Has affiliations to disclose;QLD Health:Salary/Stipend:Employment I Anne Vertigan: Has affiliations to disclose;NSW Health:Salary/Stipend:Employment I Amanda Pigott: Has affiliations to disclose;QLD Health:Salary/ Stipend:Employment I Jodie Nixon: Has affiliations to disclose;QLD Health:Salary/Stipend:Employment I Chris Wratten: Has affiliations to disclose;NSW Health:Salary/Stipend:Employment I May Boggess: Has affiliations to disclose;Arizona State University:Salary/ Stipend:Employment.

Relevant Non-financial Relationships: Claire Jeans: Has a Non-Financial Disclosure Affliation;Speech Pathology Australia membership:Professional:Membership;Australia and New Zealand Head and Neck Cancer Society:Professional:Membership I Bena Brown: Has a Non-Financial Disclosure Affliation;Australia and New Zealand Head and Neck Cancer Society:Professional:Membership I Elizabeth Ward: Has a Non-Financial Disclosure Affliation;Speech Pathology Australia:Professional:Membership;Dysphagia Research Society:Professional:Membership | Anne Vertigan: Nothing to Disclose I Amanda Pigott: Has a Non-Financial Disclosure Affliation;University of Queensland:Professional: Other volunteer activities;Australasian Lymphology Association:Professional:Membership;Occupational Therapy Australia:Professional:Membership । Jodie Nixon: Has a Non-Financial Disclosure Affliation;Child Occupational Self-Assessment:Professional:Membership I Chris Wratten: Nothing to Disclose I May Boggess: Nothing to Disclose.

\section{Statistical Power and Swallowing Rehabilitation Research: Current Landscape and Next Steps}

\section{James C. Borders $^{1}$, Michelle S. Troche ${ }^{1}$}

Purpose: Statistical power is the probability of rejecting the null when an effect exists and is an important construct to consider when designing treatment studies. A recent review found that only $9 \%$ of deglutition research reported a power analysis, suggesting that studies may be underpowered to detect a true effect. In fact, studies testing dysphagia rehabilitation paradigms often yield mixed results with non-significant changes to the penetration-aspiration scale (PAS). This study aimed to examine the current landscape of statistical power in swallowing rehabilitation research, specifically as it is related to the PAS. This was accomplished by reviewing available dysphagia treatment studies that utilized the PAS as an outcome of interest and calculating the minimum effect size that could be reliably detected with $80 \%$ statistical power for each study.

Method(s): Databases were searched for five treatments (expiratory muscle strength training, neuromuscular electrical stimulation, lingual strengthening, repetitive transcranial magnetic stimulation, head lift) using instrumental evaluations of swallowing and the PAS as an outcome. Sensitivity power analyses were performed based on the statistical test and sample size in order to determine the minimum effect size detectable with $80 \%$ power.

Result(s): Twenty-five studies were included. The median detectable effect size with $80 \%$ power was $d=1.05$ (range 0.51-2.54). No studies were powered to detect "small" effect sizes $(d<0.5)$ and only $29 \%$ were powered to detect a "moderate" effect size $(d<0.8)$.

Conclusions (Including Clinical Relevance): Dysphagia treatments examining the PAS were largely underpowered to reliably detect "small-to-moderate" effect sizes, suggesting that non-significant findings may be related to low statistical power. These findings highlight the need for collaborative, well-powered intervention studies that can detect smaller changes in swallowing function which are regarded as clinically significant. Other considerations include performing post-hoc sensitivity power analyses, determining the smallest effect size of interest, and using statistical tests that incorporate multiple trials to increase power.

Relevant Financial Relationships: James Borders: Nothing to Disclose I Michelle Troche: Has affiliations to disclose;Dysphagia Research Society:Honoraria:Other Activities;MedBridge Inc:Royalty:Other Activities;Teachers College, Columbia University:Salary/ Stipend:Employment;Michael J Fox Foundation:Grant:Independent contractor (Including contracted research);National Ataxia Foundation:Grant:Independent contractor (Including contracted research).

Relevant Non-financial Relationships: James Borders: Has a NonFinancial Disclosure Affliation;Dysphagia Research Society:Professional:Membership | Michelle Troche: Has a Non-Financial Disclosure Affliation;Dysphagia Research Society:Professional:Membership.

\section{Dysphagia Patient-Reported Outcomes in Multiple Sclerosis}

\section{Madeline Sheppard-Marvin ${ }^{1}$, Corinne A. Jones ${ }^{1}$}

Purpose: Dysphagia in multiple sclerosis (MS) is poorly characterized. The Dysphagia in Multiple Sclerosis (DYMUS) is a dysphagia questionnaire developed specifically for MS, but it lacks validation and recent research has questioned its accuracy as a screening tool. The Sydney Swallow Questionnaire (SSQ) is a validated visual analogue scale but has not been used in persons with MS. We aimed to capture patient reported swallowing measures in a large group of persons with MS and compare dysphagia complaints with other MS symptoms.

Method(s): Five hundred and thirteen adults with MS (mean age = $51.92 \pm 12.5$ years; 411 female) completed an online survey consisting of the DYMUS, SSQ, Modified Fatigue Impact Scale (MFIS), and Multiple Sclerosis Impact Scale (MSIS-29). Spearman's rank correlation and logistic regression were performed. We hypothesized there would significant relationships between the SSQ and DYMUS, and that disease duration, MFIS, and MSIS-29 would predict abnormal scores on the SSQ and DYMUS.

Result(s): Average total scores were DYMUS: 3.12/10 (SD = 2.98), SSQ: $280.13 / 1700$ (SD = 285.49), MFIS: $42 / 84(\mathrm{SD}=19.92)$, and MSIS: $75.06 / 145$ (SD = 24.94). $49.7 \%$ of respondents had an abnormal DYMUS ( $>3$ ) and $38.4 \%$ of respondents had an abnormal SSQ ( $>234) .76 .9 \%$ of respondents with abnormal DYMUS scores and $64.2 \%$ of respondents with abnormal SSQ scores reported never having received an instrumented swallowing evaluation. DYMUS and SSQ scores were significantly correlated $(r=0.83, p<0.001)$.

Abnormal DYMUS score was best described by total MFIS (OR $1.0546, p<0.001)$. Abnormal SSQ score was best described by total MFIS (OR 1.033, $p<0.001$ ), total MSIS (OR 1.02, $p<0.01$ ), and sex (OR (male) $0.44, p<0.01$ ). 
Conclusions (Including Clinical Relevance): A high proportion of respondents with complaints of dysphagia had not undergone an instrumented swallow study, suggesting that swallowing concerns are not being evaluated and treated. Furthermore, the DYMUS showed strong convergent validity to the SSQ. The DYMUS is shorter and easier to complete than the SSQ and may be suited as a screener, but further validation is warranted. Abnormal DYMUS and SSQ scores were best described using a combination of questionnaires including the MFIS, suggesting that fatigue should be evaluated and considered in assessment and management of dysphagia. More widespread use of dysphagia questionnaires, including the introduction of electronic versions, is recommended.

Relevant Financial Relationships: Madeline Sheppard-Marvin: Nothing to Disclose I Corinne Jones: Nothing to Disclose.

Relevant Non-financial Relationships: Madeline Sheppard-Marvin: Nothing to Disclose I Corinne Jones: Has a Non-Financial Disclosure Affliation;Dysphagia Research Society:Professional:Membership.

\section{Functional Outcomes Following Surgical Intervention for Osteoradionecrosis of the Mandible Following Radiation for Head and Neck Cancer}

\section{Grainne Brady ${ }^{1}$, Lauren Leigh-Doyle ${ }^{1}$, Francesco Riva ${ }^{2}$, Cyrus Kerawala $^{2}$, Justin Roe ${ }^{1,3,4}$}

Purpose: Despite recent advances in the radiation techniques used for the treatment of head and neck cancer (HNC) including intensitymodulated radiotherapy (IMRT), mandibular osteoradionecrosis (ORN) remains a significant complication. ORN can cause significant pain. Functional decline with regard to speech/swallowing may also occur on background of already compromised function from previous treatments. Pathologic fractures and oro-cutaneous fistula formations can also occur. Advanced stage ORN is managed surgically with segmental resection and immediate microvascular reconstruction. An evaluation of the functional speech and swallowing outcomes was undertaken for patients undergoing surgical management of advanced ORN

Method(s): We retrospectively reviewed 10 consecutive patients, at a single, tertiary cancer centre, who underwent surgical resection management of advanced ORN with free flap reconstruction between January 2014 and December 2019. Data include the type of flap reconstruction, duration of tracheostomy, duration of hospital stay, and Performance Status Scale for Head and Neck Cancer Normalcy of Diet (PSS-NOD) and speech intelligibility (PSS-speech) scores at baseline and 3 months following surgery.

Result(s): The sample of patients included 6 males and 4 females. Average age was 68.5 years (range 56-81). Flap reconstructions included anterolateral thigh $(n=1)$, fibular $(n=8)$, deep circumflex iliac artery (DCIA) flap $(n=1)$. Tracheostomy was required for 2 patients with median time to decannulation at 4 days (range 3-5 days). All patients were able to tolerate oral fluids on day 1 postsurgery. Two patients required supplemental nutrition via a gastrostomy at three months postsurgery. Median length of hospital stay was 9 days (range 7-15). Mean PSS-NOD score at baseline was 55 (95\% CI 44-66) and 56 at three months postoperatively (95\% CI 34.5-77.4). Eight patients had PSS-Speech at 100 and 2 patients had PSS-Speech: 75 at baseline. At 3 months postsurgery, only 6 patients had PSS-Speech: 100, whereas 4 were had PSS-Speech: 75.

Conclusions (Including Clinical Relevance): Following surgery for advanced ORN, a decline in speech and increased gastrostomy use was noted. Given the risk of baseline dysphagia in the setting of previous radiation treatment, detailed, multidimensional pre-operative assessment is required. Proactive postoperative rehabilitation should be implemented to optimise function.
Relevant Financial Relationships: Grainne Brady: Nothing to Disclose I Lauren Leigh-Doyle: Nothing to Disclose I Francesco Riva: Nothing to Disclose I Cyrus Kerawala: Nothing to Disclose I Justin Roe: Nothing to Disclose.

Relevant Non-financial Relationships: Grainne Brady: Nothing to Disclose I Lauren Leigh-Doyle: Nothing to Disclose I Francesco Riva: Nothing to Disclose I Cyrus Kerawala: Nothing to Disclose I Justin Roe: Nothing to Disclose.

\section{Swallowing Impairment Profiles in Individuals with ALS}

Lauren DiBiase $^{1,5}$, Justine Dallal York ${ }^{1,5}$, Shelby Lawrence ${ }^{2,5}$, Kayla Croft $^{2,5}$, Tara Segalewitz ${ }^{2,5}$, Shauna Mackey ${ }^{2,5}$, Grace Vernon $^{2,5}$, Krista Boesing ${ }^{2,5}$, Kelly Leonard ${ }^{2,5}$, Amber Anderson $^{1,5}$, Nicole E. Herndon ${ }^{3,2}$, Emily K. Plowman ${ }^{4,5}$

Purpose: Although it is well known that people with ALS (pALS) develop dysphagia, underlying pathophysiologic impairment profiles have not been comprehensively studied. We therefore aimed to (1) delineate oropharyngeal swallowing impairment profiles and (2) examine relationships between age, disease duration, and ALS onsettype with swallowing impairment in a large group of pALS.

Method(s): 108 pALS attended a single-assessment session and underwent a standardized videofluoroscopic swallowing exam and completed the ALS Functional Rating Scale (ALSFRS-R). Blinded duplicate ratings of the MBSImP ${ }^{\mathrm{TM}}$ were completed $(100 \%$ agreement required). Descriptives, Spearman's Rho, and ANOVA analyses were performed.

Result(s): Mean age was 65 years (SD 10.4), 50\% were male, and mean ALSFRS-R was 34 (SD 7.8). Prevalence of oral component impairment included (in rank order): lingual motion (99.9\%), swallow initiation $(97 \%)$, oral residue $(93 \%)$, bolus hold $(91 \%)$, mastication $(87 \%)$, and lip closure $(38 \%)$. Pharyngeal component impairments included laryngeal elevation (100\%), anterior hyoid excursion (98\%), pharyngeal stripping wave $(98 \%)$, pharyngeal residue $(90 \%)$, PES opening $(89 \%)$, tongue base retraction $(84 \%)$, laryngeal vestibule closure (81\%), epiglottic movement (54\%), and soft palate elevation (9\%). Mean Oral Impairment Total (OT) and Pharyngeal Impairment Total (PT) scores were 11.4/22 (SD 2.9) and 9.9/26 (SD 3.1), respectively. Age was associated with both OT and PT scores $(r=0.2, p<0.05)$. No associations were revealed between swallowing impairment and disease duration or global disease progression $(p>0.05)$. Significant positive associations were, however, noted between OT and PT scores with bulbar disease progression $(p<0.001)$. Finally, both OT and PT were significantly higher (worse) for patients with a bulbar-onset disease type $(p<0.005)$.

Conclusions (Including Clinical Relevance): In this cohort of 108 pALS, oropharyngeal swallowing impairment was associated with older age, greater bulbar disease progression, and bulbar-onset disease. While both oral and pharyngeal impairments occurred, oral phase swallowing deficits were more prevalent and more severe.

Relevant Financial Relationships: Lauren DiBiase: Has affiliations to disclose;University of Florida:Salary/Stipend:Employment I Justine Dallal York: Has affiliations to disclose;University of Florida:Salary/ Stipend:Employment I Shelby Lawrence: Has affiliations to disclose;University of Florida:Salary/Stipend:Employment | Kayla Croft: Nothing to Disclose I Tara Segalewitz: Nothing to Disclose I Shauna Mackey: Nothing to Disclose I Grace Vernon: Nothing to Disclose I Krista Boesing: Nothing to Disclose I Kelly Leonard: Has affiliations to disclose;University of Florida:Salary/Stipend:Employment I Amber Anderson: Has affiliations to disclose;University of Florida:Salary/Stipend:Employment I Nicole Herndon: Has affiliations to disclose;University of Florida Health:Salary/ 
Stipend:Employment | Emily Plowman: Has affiliations to disclose;University of Florida:Salary/ Stipend:Employment;Biohaven:Consulting fee:Consulting;ALS Association:Grant:Other Activities;National Institutes of Health:Grant:Other Activities.

Relevant Non-financial Relationships: Lauren DiBiase: Has a NonFinancial Disclosure Affliation;American Speech-Language-Hearing Association:Professional:Membership | Justine Dallal York: Has a Non-Financial Disclosure Affliation;Dysphagia Research Society:Professional:Membership;American Speech-Language-Hearing Association:Professional:Membership I Shelby Lawrence: Has a NonFinancial Disclosure Affliation;American Speech-Language-Hearing Association:Professional:Membership | Kayla Croft: Nothing to Disclose I Tara Segalewitz: Has a Non-Financial Disclosure Affliation;American Speech-Language-Hearing Association:Professional:Membership I Shauna Mackey: Has a Non-Financial Disclosure Affliation;American Speech-Language-Hearing Association:Professional:Membership I Grace Vernon: Has a Non-Financial Disclosure Affliation;American Speech-Language-Hearing Association:Professional:Membership I Krista Boesing: Has a Non-Financial Disclosure Affliation;American Speech-Language-Hearing Association:Professional:Membership I Kelly Leonard: Has a Non-Financial Disclosure Affliation;American Speech-Language-Hearing Association:Professional:Membership I Amber Anderson: Has a Non-Financial Disclosure Affliation;American Speech-Language-Hearing Association:Professional:Membership I Nicole Herndon: Has a Non-Financial Disclosure Affliation;American Speech-Language-Hearing Association:Professional:Membership I Emily Plowman: Has a Non-Financial Disclosure Affliation;Dysphagia Research Society:Professional:Membership;American Speech-Language-Hearing Association:Professional:Membership;Northeast ALS Consortium (NEALS):Professional:Volunteer membership on advisory committee or review panels.

\section{Effects of Physiochemical Properties of Food and Liquids on Swallowing Physiology in Adults: A Systematic Review}

Rodolfo E. Pena ${ }^{1,2,3,8}$, Nicole Schaen-Heacock ${ }^{4}$, Mary Hitchcock ${ }^{5}$, Atsuko Kurosu $^{2,6}$, Michelle R. Ciucci ${ }^{1,6,9}$, Nicole Rogus-Pulia ${ }^{2,7,8}$

Purpose: Physiochemical properties of food and liquids provide sensory input that can modify swallowing physiology in both healthy adults and patients with dysphagia. The purpose of this systematic review was to understand which specific properties of food, and liquids are associated with changes in swallowing physiology in adults with and without dysphagia.

Method(s): Six databases were systematically searched by two reviewers from inception through January 15th of 2020 to identify relevant studies that included only human subjects, instrumental assessment of the swallowing, and both healthy adults and patients with dysphagia. Full-text reviews of abstracts that met inclusion criteria were completed, and relevant data were extracted independently. Result(s): The search yielded 3344 unique abstracts. Of the 160 abstracts that underwent full-text review, 75 were accepted. 3 of these studies were randomized controlled trials and 72 were non-randomized including cohort, case-control, cross-sectional, and longitudinal studies. Results of 69 studies showed an influence of physiochemical bolus properties on swallowing physiology. Specific properties found to be associated with changes in various aspects of swallowing physiology included viscosity (150-3931cp; $37 / 41$ studies); taste (sour, sweet, and salty; 22/26 of studies); texture (hardness; 8/8 studies); and chemesthesis (natural capsaicin and carbonation; 11/11 studies). Study population, study inclusion criteria, and method for quantifying change in swallowing physiology varied among studies. 48 studies focused on healthy adults and 27 on patients with dysphagia. For swallowing assessment, the following instrumental methods were used in the included studies: videofluoroscopy (29), surface electromyography (15), tongue pressure measurement (11), fiberoptic endoscopic evaluation of swallowing (8), pharyngeal highresolution manometry (3), scintigraphy (5), computerized tomography (1), electromagnetic midsagittal articulography (2), and ultrasonography (1).

Conclusions (Including Clinical Relevance): Variations in the viscosity, taste, texture, and chemesthetic properties of solids and liquids were found to modify swallowing physiology in both healthy adults and patients with dysphagia. These data will be critical as a foundation for scientists and clinicians to collaboratively develop beverages and foods that optimize swallowing function for patients with dysphagia.

Relevant Financial Relationships: Rodolfo Pena: Has affiliations to disclose;Department of Medicine, UW-Madison:Salary/Stipend:Employment I Nicole Schaen-Heacock: Has affiliations to disclose;University of Wisconsin-Madison:Salary/Stipend:Employment;National Institute of Health:Salary/Stipend:Employment I Mary Hitchcock: Has affiliations to disclose;University of WisconsinMadison:Salary/Stipend:Employment I Atsuko Kurosu: Has affiliations to disclose;University of Wisconsin-Madison:Salary/ Stipend:Employment;National Institute of Health:Salary/ Stipend:Employment | Michelle Ciucci: Has affiliations to disclose;University of Wisconsin-Madison:Salary/ Stipend:Employment;National Institute of Health:Grant:Employment I Nicole Rogus-Pulia: Has affiliations to disclose;University of Wisconsin-Madison:Salary/Stipend:Employment;National Institute of Health:Grant:Employment;William S. Middleton Memorial Veterans Hospital:Salary/Stipend:Employment;William S. Middleton Memorial Veterans Hospital:Grant:Employment;University of Wisconsin Medical Foundation:Salary/Stipend:Employment.

Relevant Non-financial Relationships: Rodolfo Pena: Has a NonFinancial Disclosure Affliation;Dysphagia Research Society:Professional:Membership | Nicole Schaen-Heacock: Has a Non-Financial Disclosure Affliation;Dysphagia Research Society:Professional:Membership I Mary Hitchcock: Nothing to Disclose I Atsuko Kurosu: Has a Non-Financial Disclosure Affliation;Dysphagia Research Society:Professional:Membership | Michelle Ciucci: Has a Non-Financial Disclosure Affliation;National Foundation of Swallowing Disorders:Professional:Board membership;Dysphagia Research Society:Professional:Board membership | Nicole RogusPulia: Has a Non-Financial Disclosure Affliation;Dysphagia Research Society:Professional:Board membership.

\section{Exploring Patient Experiences with a TeleHealth Approach for the PRO-ACTIVE Trial Intervention in Head and Neck Cancer Patients}

Maisha M. Khan ${ }^{1,2}$, Beatrice Manduchi ${ }^{1,3}$, Veronica Rodriguez ${ }^{1,2}$, $\overline{\text { Margaret I. Fitch }}{ }^{4}$, Katherine A. Hutcheson ${ }^{5}$, Rosemary Martino $^{1,2,3,6}$

Purpose: PRO-ACTIVE Trial patients receive bi-weekly in-person SLP intervention regarding dysphagia management during radiotherapy for head and neck cancer. Following the COVID-19 directive to cease non-essential services, the trial rapidly shifted to provide dysphagia management via telehealth $(\mathrm{TH})$. This study explored patient experiences with the TH modality for PRO-ACTIVE intervention.

Method(s): Patients who experienced the TH approach participated in a one-time semi-structured interview. Interviews were audiotaped 
using MS teams, transcribed, and subjected to a standard qualitative content/theme analysis. Trained reviewers coded all interview transcripts using the framework developed from initial review of $20 \%$ of the data. Coded response categories were independently reviewed and key messages identified through consensus discussion.

Result(s): Seven of ten patients using the TH approach were interviewed. All experienced sessions where an SLP observed their swallowing, discussed the EAT staircase and, depending on treatment arm, trained swallowing exercises. Overall, patients considered the use of TH as great but some were not sure what to expect before the session. Most felt sessions worked well, and reported receiving clear instruction and guidance. A few patients expressed concerns regarding internet connections. Most reported it was helpful to have a caregiver present, an engaging clinician lead the sessions and have previous experience using teleconferencing even if it was not related to healthcare. Only a few patients reported not having access to optimal equipment, technical knowledge, or caregiver support. No barriers were identified preventing participation. Overall patients reported $\mathrm{TH}$ as beneficial as it saved commuting time and allowed caregiver participation. During COVID-19, they reported the added benefit of continued research participation while reducing risk of exposure. Most patients found TH sessions just as effective as inperson sessions. The only drawback identified was that teleconferencing did not allow a close physical examination. Patients indicated that video allows SLPs to demonstrate exercises and provide feedback and is, therefore, better than audio alone.

Conclusions (Including Clinical Relevance): Patients reported $\mathrm{TH}$ sessions did not compromise with what they would have learnt in inperson sessions. Patient feedback about TH will inform best practices in utilizing this approach for SLP care delivery.

Relevant Financial Relationships: Maisha Khan: Nothing to Disclose I Beatrice Manduchi: Nothing to Disclose I Veronica Rodriguez: Nothing to Disclose I Margaret Fitch: Nothing to Disclose I Katherine Hutcheson: Nothing to Disclose I Rosemary Martino: Nothing to Disclose.

Relevant Non-financial Relationships: Maisha Khan: Nothing to Disclose | Beatrice Manduchi: Nothing to Disclose | Veronica Rodriguez: Nothing to Disclose I Margaret Fitch: Nothing to Disclose I Katherine Hutcheson: Nothing to Disclose I Rosemary Martino: Nothing to Disclose.

\section{Dysphagia in Lung Transplant Recipients: Prevalence, Risk Factors, and Health-Related Outcomes}

\section{Justine Dallal York ${ }^{1,5}$, Jennifer Colsky ${ }^{2}$, Kayla Croft ${ }^{3}$, Tara Segalewitz ${ }^{3}$, Tiago Machuca ${ }^{4}$, Lauren DiBiase ${ }^{1,5}$, Amber Anderson ${ }^{1,5}$, Emily K. Plowman ${ }^{6,5}$}

Purpose: Although lung transplantation (LT) remains the definitive therapy for end-stage respiratory disease, current 5-year survival rates of $\sim 56 \%^{1-3}$ highlight the need for improved postoperative care. ${ }^{4}$ Given the high risk for postoperative dysphagia, we aimed to determine the prevalence, risk factors, and associated outcomes of dysphagia in postoperative LT patients.

Method(s): A retrospective review of consecutive patients undergoing LT and postoperative videofluoroscopic swallowing evaluations (VFSS) at UF Health between 11/2017 and 6/2020 was conducted. Relevant outcomes were extracted from electronic medical records (EPIC) and entered into a central database (REDCap). VFSS notes were examined and Penetration Aspiration Scale (PAS) scores extracted. Descriptives, t tests, one-way ANOVA's, Chi squares, and odds ratios were derived in SPSS

Result(s): 205 LT recipients met inclusion criteria and were included. Mean age was 58.6 years and $51 \%$ were male. Safe swallowing was confirmed in $20 \%(n=41)$, laryngeal penetration in $40 \%(n=82)$, and tracheal aspiration in $40 \%(n=82)$. Aspiration response profiles included $50 \%$ no cough response $(n=41), 31.7 \%$ ejection of aspirate material $(n=26)$, and $18.3 \%$ ineffective cough response $(n=15)$. Aspiration in LT patients was associated with (1) venous-venous extracorporeal membrane oxygenation (OR 2.0, $X_{2}=3.7, p=0.05$ ); (2) cardiopulmonary bypass (OR $2.3, X_{2}=5.7, p=0.02$ ); (3) $\geq 3$ intubations (OR 7.5, $\left.X_{2}=5.2, p=0.02\right)$; (4) presence of tracheotomy (OR 4.8, $\left.X_{2}=8.3, \mathrm{p}=0.004\right)$; and (5) longer intubation duration (70 vs. $44 \mathrm{~h}, p=0.002$ ). LT patients who aspirated waited an average of $240 \mathrm{~h}$ longer to resume a regular diet $(p=0.004)$ and demonstrated a 2.4 and 2.0 higher odds of pneumonia and discharge to a non-home setting, respectively. One-year mortality rate was $2.9 \%(n=6)$, with $100 \%$ classified as unsafe swallowers.

Conclusions (Including Clinical Relevance): Dysphagia was prevalent and associated with reduced patient outcomes. The observation that $\sim 70 \%$ of aspirators could not clear tracheal aspirate may explain, in part, the increased odds for development of pneumonia. Mortality data suggest that presence of dysphagia may impact longterm survival and will be further examined in ongoing prospective studies.

Relevant Financial Relationships: Justine Dallal York: Nothing to Disclose I Jennifer Colsky: Nothing to Disclose I Kayla Croft: Nothing to Disclose I Tara Segalewitz: Nothing to Disclose I Tiago Machuca: Nothing to Disclose I Lauren DiBiase: Nothing to Disclose I Amber Anderson: Nothing to Disclose I Emily Plowman: Has affiliations to disclose;University of Florida:Salary/Stipend:Employment;Biohaven:Consulting fee:Consulting;ALS Association:Grant:Other Activities;National Institute of Health:Grant:Other Activities.

Relevant Non-financial Relationships: Justine Dallal York: Has a Non-Financial Disclosure Affliation;DRS:Professional:Membership I Jennifer Colsky: Nothing to Disclose I Kayla Croft: Nothing to Disclose I Tara Segalewitz: Has a Non-Financial Disclosure Affliation;ASHA:Professional:Membership I Tiago Machuca: Nothing to Disclose | Lauren DiBiase: Nothing to Disclose I Amber Anderson: Nothing to Disclose I Emily Plowman: Has a Non-Financial Disclosure Affliation;DRS:Professional:Membership.

\section{Diversity of Feeding Diet and Its Relation to Communicative Development at 18 Months}

\section{Alaina Martens $^{1}$, Emily Zimmerman ${ }^{1}$}

Purpose: The goal of the proposed research is to determine the association between diversity of feeding diet and communicative development in full-term infants at 18 months of age. This comparison will help guide clinicians' use of feeding therapies and mealtimes as opportunites for speech and language development. Additionally, it will encourage caregivers to use feeding as an opportunity for rich language exposure.

Method(s): This study examines a cohort of full-term infants $(n=20)$ at 18 months of age. These data are taken from a larger ongoing study examining the relation between sucking, feeding and vocal development. Participants were recruited through flyers, word of mouth and by posting in parent Facebook groups. Caregivers completed a Qualtrics survey to examine feeding and communication development beyond the first year of life. This survey included a Food Inventory, which consisted of a list of potential foods the infant had been exposed to, which were separated into six categories (protein, carbs/grains, fruits/vegetables, dairy/eggs, legumes/nuts, finger foods). Additional space was provided for caregivers to add foods not previously mentioned. Parents also completed the MacArthur-Bates Communication Development Inventory (CDI). Participants received a $\$ 10$ Amazon gift card for completion of the survey. 
Result(s): Twenty full-term infants at 18 -months of age were included. CDI data were not normalized; therefore, Spearman Correlations were calculated (see data below, CDI variables on left).

Phrases Understood 0.326

Comprehension of Words \& Phrases +0.441 .

Words Understood 0.212 .

Words Produced 0.036

Early Gestures 0.296 .

Late Gestures 0.113.

Total Gestures 0.189 .

Conclusions (Including Clinical Relevance): Overall, these correlations show a positive interaction between Food Inventory and the CDI scales. Comprehension of Words and Phrases had a nearly significant strong interaction with food inventory ( $\mathrm{rs}=0.441, p=0.05$ ), indicating that children who comprehended more words and phrases showed a strong trend of exposure to a wider variety of foods. Similar medium trends were seen between phrases understood and words understood.

Findings from this work highlight mealtime and feeding therapies as a prime context to encourage speech and language development. The proposed study is likely to increase evidence for developmental specialists in early intervention to target communication and feeding goals in tandem.

Relevant Financial Relationships: Alaina Martens: Nothing to Disclose I Emily Zimmerman: Has affiliations to disclose;Northeastern University:Salary/Stipend:Employment.

Relevant Non-financial Relationships: Alaina Martens: Has a NonFinancial Disclosure Affliation;DRS:Professional:Membership | Emily Zimmerman: Has a Non-Financial Disclosure Affliation;DRS:Professional:Membership.

\section{Relationship Between Scores on the Child Oral and Motor Proficiency Scale at 12 months and Non- nutritive Sucking at 3 Months}

\section{Morgan E. Hines $^{1}$, Alaina Martens ${ }^{1}$, Emily Zimmerman ${ }^{1}$}

Purpose: The goal of this study was to characterize the relationship between infants' Non-Nutritive Sucking (NNS) patterns at 3 months and scores on the Child Oral and Motor Proficiency Scale (ChOMPS). Given that the ChOMPS examines more complex oral motor movements, we hypothesized that infants' NNS at 3 months would be significantly associated with oral and motor profiency at 12 months. Method(s): To measure NNS, infants sucked on our custom research pacifier for $\sim 5 \mathrm{~min}$ at 3 months of age, yielding the following NNS dependent measures: duration (s), amplitude $\left(\mathrm{cmH}_{2} \mathrm{O}\right)$, frequency $(\mathrm{Hz})$, bursts per minute, cycles per burst, and cycles per minute.

Result(s): Thus far, 52 full-term infants (30 males) have NNS sampled and ChOMPS completed in this ongoing study. The Spearman correlations revealed significant associations between scores for the ChOMPS Oral Motor Coordination domain and NNS duration ( $\mathrm{rs}=$ $-0.324, p=0.020$ ), Complex Movement domain and NNS bursts ( $\mathrm{rs}=-0.385, p=0.005)$ and cycles per minute ( $\mathrm{rs}=-0.297$, $p=0.032$ ). The ChOMPS total Score and NNS bursts ( $\mathrm{rs}=-0.301$, $p=0.030)$ and cycles per minute $(\mathrm{rs}=-0.293, p=0.035)$ at 3 months.

Conclusions (Including Clinical Relevance): These data show that better Oral Motor Coordination at 12 months was related to shorter NNS durations at 3 months. More complex movement functions were associated with fewer bursts and suck cycles per minute. Higher total oral and motor proficiency scores were related to fewer NNS bursts and suck cycles per minute. We will continue to enroll infants into this ongoing study. These preliminary findings indicate that infant suck patterns may be indicative of future oral motor coordination.
Further analyses are needed with more participants and across development in an effort to better undertstand the relation beween infant suck and subsequent oral motor development (i.e., chewing and early speech).

Relevant Financial Relationships: Morgan Hines: Has affiliations to disclose;Northeastern University:Salary/Stipend:Employment । Alaina Martens: Has affiliations to disclose;Northeastern University:Salary/Stipend:Employment I Emily Zimmerman: Has affiliations to disclose;Northeastern University:Salary/Stipend:Employment.

Relevant Non-financial Relationships: Morgan Hines: Nothing to Disclose I Alaina Martens: Nothing to Disclose I Emily Zimmerman: Nothing to Disclose.

\section{Construction of a Pediatrics Dysphagia Screening Tool}

\section{Azza Aziz ${ }^{1}$, Dalia Osman ${ }^{2}$, Asmaa Abdelhameed ${ }^{3}$, Ahmed Nagy Abdelrahman $^{5,7}$, Rasha Asheery ${ }^{4,6}$}

Purpose: The aim of this study is the early detection of swallowing and feeding problems in children by using a non-invasive screening tool to allow early intervention, rehabilitation, and prevention of dysphagia complications. The goal is to diversify the pediatric study cohort in terms of the array of disorders causing Dysphagia and to develop a comprehensive tool applicable to a wide range of patients. Method(s): This study included sixty children aged 2 to 18 years, diagnosed with a primary disorder carrying an additional risk of having Dysphagia. The initial step was general history taking from parents/caretakers to monitor the eligibility for inclusion, following this was a thorough feeding history. The next step was administering the pediatric dysphagia screening questionnaire (PDSQ). The PDSQ consists of twelve screening questions. All questions are yes or no questions. Yes is marked as 1 , and No as 0 . Subsequently, all subjects underwent a Clinical feeding assessment and a Fiberoptic Endoscopic Evaluation of Swallowing (FEES).

Result(s): All items of the PDSQ had a statistically significant higher Yes rating in dysphagic patients compared to the non Dysphagic group. We performed the Receive Operating Characteristics curve to determine the discrimination value of the total questionnaire scores for dysphagia as diagnosed with Clinical observation, Penetration \&/ or Aspiration, Penetration only, and Aspiration only as diagnosed by FEES. We plotted the cut-off points for different levels of sensitivity and specificity. A cut-off point of 2.5 revealed to have adequate sensitivity of $97.9 \%$ and specificity of $100 \%$ to detect dysphagia diagnosed by clinical observation. 6.5 is the cut off to detect Penetration \&/or aspiration in FEES with a sensitivity of $85.2 \%$ and specificity of $81.8 \%$. Also, a 6.5 cut off revealed to have a sensitivity of $83.3 \%$ and specificity of $80.6 \%$ to detect Penetration in FEES, and 8.5 is the most acceptable cut-off point to predict aspiration in FEES with a sensitivity of 84.2 and a specificity of 85.4.

Conclusions (Including Clinical Relevance): The analysis shows that the tool could be a potentially valuable contribution to clinical practice.The broadness of the study population and the simplicity of using the tool advocate for its value as a possible screening alternative for use in children with disorders associated with dysphagia as comorbidity.

Relevant Financial Relationships: Azza Aziz: Has affiliations to disclose;Cairo University:Salary/Stipend:Employment I Dalia Osman: Has affiliations to disclose;Cairo University:Salary/Stipend:Employment I Asmaa Abdelhameed: Has affiliations to disclose;Cairo University:Salary/Stipend:Employment I Ahmed Nagy Abdelrahman: Has affiliations to disclose;University at Buffalo:Salary/Stipend:Employment I Rasha Asheery: Has affiliations to disclose;Fayoum University:Salary/Stipend:Employment. 
Relevant Non-financial Relationships: Azza Aziz: Nothing to Disclose I Dalia Osman: Nothing to Disclose I Asmaa Abdelhameed: Nothing to Disclose I Ahmed Nagy Abdelrahman: Nothing to Disclose I Rasha Asheery: Nothing to Disclose.

\section{Incomplete Glottic Closure and Post-Swallow Residue are Associated with Aspiration in Cardiovascular Surgical Patients}

\section{Emily K. Plowman ${ }^{1,2}$, Justine Dallal York ${ }^{2,3}$, Amber Anderson ${ }^{2,3}$, Lauren DiBiase $^{2,3}$, Tara Segalewitz ${ }^{2,4}$, Kayla Croft ${ }^{2,4}$, Eric Jeng ${ }^{5}$, Neil Chheda ${ }^{6}$}

Purpose: We have previously reported a high rate of aspiration in postoperative cardiovascular patients that is associated with increased length of hospital stay, cost of care, pneumonia, reintubation, and death. ${ }^{1}$ Currently, contributing physiologic mechanisms of unsafe swallowing are not known, limiting the development of targeted interventions. Given this gap in knowledge, we sought to examine contributing physiologic factors of aspiration in postoperative cardiovascular patients.

Method(s): A prospective single-center study was conducted in postoperative adult cardiovascular patients between February 2019 and January 2020. Consenting patients underwent a fiberoptic endoscopic evaluation of swallowing within $72 \mathrm{~h}$ of extubation. Two independent and blinded raters assessed swallowing safety and efficiency using the penetration aspiration scale (PAS) and Yale Residue Rating Scale, respectively. Blinded ratings of vocal fold mobility impairment were assessed by a laryngologist. Independent $t$ tests, Chi squares, and odds ratios (OR) were performed with alpha set at 0.05 . Result(s): 200 individuals participated in this study and $56(28 \%)$ were confirmed aspirators. Vocal fold paralysis was not associated with aspiration $\left(X^{2}=0.79, p=0.37\right)$, with noted compensation of the intact vocal fold to protect the airway. Glottic closure competency was associated with aspiration status $\left(X^{2}=6.3, p=0.01\right)$. Patients with incomplete glottic closure demonstrated a 3.8 (95\% CI 1.3, 11.6) higher odds of aspiration. Post swallow residue was also associated with aspiration $\left(X^{2}=29.4, p<0.001\right)$. Patients with pharyngeal residue demonstrated a $6.7(95 \%$ CI $3.2,14.1)$ higher odds of aspiration.

Conclusions (Including Clinical Relevance): The inability to achieve complete glottic closure and presence of post-swallow pharyngeal residue were associated with aspiration in this group of cardiovascular patients. While the former mechanism relates to the ability to seal the true vocal folds as a critical step for closure of the larynx, we believe the latter represents a secondary contributing mechanism increasing risk of aspiration on subsequent swallows.

Relevant Financial Relationships: Emily Plowman: Has affiliations to disclose;University of Florida:Salary/Stipend:Employment;Biohaven:Salary/Stipend:Consulting;ALS Association:Grant:Other Activities;National Institutes of Health:Grant:Other Activities I Justine Dallal York: Has affiliations to disclose;University of Florida:Salary/Stipend:Employment I Amber Anderson: Has affiliations to disclose;University of Florida:Salary/Stipend:Employment I Lauren DiBiase: Has affiliations to disclose;University of Florida:Salary/Stipend:Employment | Tara Segalewitz: Nothing to Disclose | Kayla Croft: Nothing to Disclose I Eric Jeng: Has affiliations to disclose;University of Florida:Salary/Stipend:Employment; University of Florida Department of Surgery New Investigator Award:Grant:Other Activities I Neil Chheda: Has affiliations to disclose;University of Florida:Salary/Stipend:Employment.

Relevant Non-financial Relationships: Emily Plowman: Has a NonFinancial Disclosure Affliation;Dysphagia Research Society:Professional:Membership;American
Association:Professional:Membership;Northeast ALS Consortium (NEALS):Professional:Volunteer membership on advisory committee or review panels I Justine Dallal York: Has a Non-Financial Disclosure Affliation;Dysphagia

Research Society:Professional:Membership;American Speech-LanguageHearing Association:Professional:Membership | Amber Anderson: Has a Non-Financial Disclosure Affliation;American Speech-Language-Hearing Association:Professional:Membership | Lauren DiBiase: Has a Non-Financial Disclosure Affliation;American Speech-Language-Hearing Association:Professional:Membership Tara Segalewitz: Has a Non-Financial Disclosure Affliation;American Speech-Language-Hearing Association:Professional:Membership | Kayla Croft: Nothing to Disclose I Eric Jeng: Nothing to Disclose I Neil Chheda: Nothing to Disclose.

\section{Sarcopenia and Dysphagia Risk in Parkinson's Disease}

\section{Ana Luisa Capitelli Dornellas ${ }^{1}$, Flávia Pereira da Costa Christianini $^{1}$, Daniella Lima ${ }^{1}$, Natalia Conessa Ortega ${ }^{1}$, Adriana Ponsoni $^{1}$, Rachel Guimarães ${ }^{1}$, Lucia Mourão ${ }^{1}$}

Purpose: Sarcopenia is a condition in aging subjects exacerbated by motor impairment and loss of functional mobility in Parkinson's Disease (PD). Dysphagia, often present in PD, is also worsened by sarcopenia. Therefore, it is crucial to investigate the association between dysphagia and sarcopenia risk in PD patients through distinct risk assessment instruments and to determine their correlation with sociodemographical and clinical variables.

Method(s): Methods: 24 idiopathic PD patients answered two selfevaluation surveys for dysphagia risk: the Eating Assessment Tool (EAT-10) and the Swallowing Disturbance Questionnaire (SDQ-PD), and were subject to risk assessment instruments for sarcopenia: SARC-F, calf circumference (CC), and hand grip strength (HGS). To ascertain whether there was an increase in dysphagia risk in the group with sarcopenia risk, we used the Mann-Whitney test and the Spearman Correlation Test was used to find a possible association between age and disease duration and sarcopenia and dysphagia indicators. Significance level was set at $p<0.05$.

Result(s): Results: Mean participant age was $66.37 \pm 12.71$ years and mean disease duration was $9.7 \pm 6.56$ years. Median $\mathrm{H} \& \mathrm{Y}=2.5$ and UPDRS $=40$. Dysphagia risk was positively correlated with disease duration only in EAT-10. There was a correlation between higher UPDRS total scores and its motor subscale scores with higher SARC-F scores and lower CC and HGS measurements. When analyzing sarcopenia and dysphagia risk in elderly with PD, worse SDQPD scores were related with lower $\mathrm{CC}$ values.

Conclusions (Including Clinical Relevance): Conclusion: This study uncovered an association between dysphagia risk and sarcopenia in PD. Lower UPDRS scores correlated with higher sarcopenia risk for all risk assessment instruments. Dysphagia risk presented variable results depending on the instrument and likelihood of sarcopenia in PD patients, calling for new studies to explore further how sarcopenia impacts swallowing in the PD population.

Relevant Financial Relationships: Ana Luisa Capitelli Dornellas: Nothing to Disclose I Flávia Pereira da Costa Christianini: Nothing to Disclose | Daniella Lima: Nothing to Disclose | Natalia Conessa Ortega: Nothing to Disclose I Adriana Ponsoni: Nothing to Disclose I Rachel Guimarães: Nothing to Disclose I Lucia Mourão: Nothing to Disclose.

Relevant Non-financial Relationships: Ana Luisa Capitelli Dornellas: Nothing to Disclose I Flávia Pereira da Costa Christianini: Has a Non-Financial Disclosure Affliation;DRS:Professional:Membership | Daniella Lima: Nothing to Disclose | Natalia Conessa Ortega: 
Nothing to Disclose I Adriana Ponsoni: Nothing to Disclose I Rachel Guimarães: Nothing to Disclose I Lucia Mourão: Has a Non-Financial Disclosure Affliation;DRS:Professional:Membership.

\section{Swallowing Disorders in Patients with Chronic Obstructive Pulmonary Disease: A Systematic Review and Meta-Analysis}

\author{
Cagla Kantarcigil ${ }^{1}$, Victoria S. McKenna ${ }^{4}$, Linda C. O'Dwyer ${ }^{2}$, \\ Lutfiyya Muhammad $^{3}$, Bonnie Martin-Harris ${ }^{1,5}$
}

Purpose: Dysphagia has been reported in up to $85 \%$ of individuals with Chronic Obstructive Pulmonary Disease (COPD). Despite the high prevalence, little attention has been given to examining dysphagia in patients with COPD. The aims of this systematic review and meta-analysis were to summarize the current evidence and critically appraise studies that examined swallowing outcomes in patients with COPD.

Method(s): A search was conducted across PubMed, MEDLINE, Embase, CINAHL, Web of Science, Scopus, ProQuest, and clinicaltrials.gov with no date limits. Studies were included if they enrolled adult patients with COPD and used instrumental assessment to examine swallowing. Studies were excluded if they were not written in English and used a single-subject research design. A mixed-effects meta-analysis of penetration/aspiration scores was conducted to compute an overall proportion of airway invasion. A modified version of the NIH Quality Assessment Tool was used to assess risk of bias. Result(s): The search identified 8949 articles, of which 18 studies met all inclusion criteria. The majority of studies used Modified Barium Swallow Study (13/18), whereas Fiberoptic Endoscopic Evaluation of Swallowing was used in 4 studies, with one study employing both methods. Results consistently showed poor bolus preparation/mastication, delayed swallow initiation, decreased laryngeal elevation and tongue base retraction, and increased oral/ pharyngeal residue in patients with COPD. Longer oral/pharyngeal transit times were also reported compared to healthy controls. Of the 15 studies that examined airway invasion, penetration/aspiration was observed in 10, with high occurrence of silent aspiration. Metaanalysis results showed a pooled aspiration rate of $34 \%(95 \% \mathrm{CI}$ $21-47 \%$ ) across 560 subjects. All studies demonstrated risk of bias, with a noted lack of blinding and reliability testing in approximately $75 \%$ of studies.

Conclusions (Including Clinical Relevance): Impairments in the swallowing mechanism, airway invasion, and residue were reported; however, studies often lacked valid and reliable metrics to quantify these impairments. The majority of studies did not account for COPD state/severity and confounding factors. Only half of the studies included a control group. Thus, a lack of rigorous research persists. We recommend future research using objective and reproducible methods in homogeneous groups to characterize impairments in those with COPD.

Relevant Financial Relationships: Cagla Kantarcigil: Has affiliations to disclose;Northwestern University:Salary/ Stipend:Employment I Victoria McKenna: Has affiliations to disclose;University of Cincinnati:Salary/Stipend:Employment I Linda O'Dwyer: Has affiliations to disclose;Northwestern University:Salary/Stipend:Employment | Lutfiyya Muhammad: Has affiliations to disclose;Northwestern University:Salary/Stipend:Employment | Bonnie Martin-Harris: Has affiliations to disclose;Northwestern University:Salary/Stipend:Employment;NIH:Grant:Other Activities;VA:Grant:Other Activities;Northern Speech Services:Royalty:Other Activities;MUSC Research Development Fund:Royalty:Other Activities.
Relevant Non-financial Relationships: Cagla Kantarcigil: Has a Non-Financial Disclosure Affliation;Dysphagia Research Society:Professional:Membership | Victoria McKenna: Nothing to Disclose I Linda O'Dwyer: Nothing to Disclose I Lutfiyya Muhammad: Nothing to Disclose I Bonnie Martin-Harris: Has a NonFinancial Disclosure Affliation;Dysphagia Research Society:Professional:Membership;American Speech-Language and Hearing Association:Professional:

Membership;ABSSD:Professional:Membership.

\section{Relationship Between Oropharyngeal Swallowing Impairment, Clinical Bulbar Metrics, and Patient Report in Individuals with ALS}

\author{
Lauren DiBiase $^{1,5}$, Justine Dallal York ${ }^{1,5}$, Kayla Croft ${ }^{2,5}$, Tara \\ Segalewitz $^{2,5}$, Shelby Lawrence ${ }^{2,5}$, Grace Vernon ${ }^{2,5}$, Krista \\ Boesing $^{2,5}$, Shauna Mackey ${ }^{2,5}$, Kelly Leonard ${ }^{2,5}$, Amber \\ Anderson $^{1,5}$, Nicole E. Herndon ${ }^{3,2}$, Emily K. Plowman ${ }^{4,5}$
}

Purpose: Although it is widely known that people with ALS (pALS) develop bulbar impairment in the form of dysphagia and/or dysarthria, little is known regarding the relationship between swallowing impairment profiles and clinical metrics of bulbar function, or patientperceived impairment. We, therefore, aimed to examine relationships between oropharyngeal swallowing impairment with bulbar strength, speech, functional oral intake, and patient self-report profiles.

Method(s): 108 pALS underwent comprehensive testing of lingual strength (IOPI), voluntary cough (PEF), the Sentence Intelligibility Test (SIT), Eating Assessment Tool-10 (EAT-10), Functional Oral Intake Scale (FOIS), and a standardized videofluoroscopic swallowing exam. Blinded duplicate ratings of the MBSImP $^{\mathrm{TM}}$ protocol were completed and Oral (OT) and Pharyngeal Impairment Total (PT) scores were derived (100\% agreement required). Descriptives and Spearman's Rho correlations were performed.

Result(s): Mean age was 65 years (SD 10.4), 50\% were male, and average disease duration was 30 months (SD 23.7). Higher (worse) $\mathrm{OT}$ and PT MBSImP ${ }^{\mathrm{TM}}$ scores were associated with higher (worse) EAT-10 $(p<0.001)$, lower FOIS $(p<0.001)$, and reduced lingual strength $(p<0.003)$. Reduced cough PEF was associated with higher OT scores $(p<0.01)$ but not associated with PT scores. Conversely, reduced speaking rate and intelligibility were associated with higher PT scores $(p<0.05)$ but not OT impairment scores.

Conclusions (Including Clinical Relevance): pALS with increased oropharyngeal swallowing impairment was noted to have reduced lingual strength, to consume a more restrictive diet, and report more advanced swallowing deficits. Reduced cough strength was associated with confirmed oral phase swallowing impairment while increased dysarthria was associated with pharyngeal phase impairment.

Relevant Financial Relationships: Lauren DiBiase: Has affiliations to disclose;University of Florida:Salary/Stipend:Employment I Justine Dallal York: Has affiliations to disclose;University of Florida:Salary/ Stipend:Employment I Kayla Croft: Nothing to Disclose I Tara Segalewitz: Nothing to Disclose I Shelby Lawrence: Has affiliations to disclose;University of Florida:Salary/Stipend:Employment I Grace Vernon: Nothing to Disclose I Krista Boesing: Nothing to Disclose I Shauna Mackey: Nothing to Disclose I Kelly Leonard: Has affiliations to disclose;University of Florida:Salary/Stipend:Employment I Amber Anderson: Has affiliations to disclose;University of Florida:Salary/ Stipend:Employment I Nicole Herndon: Has affiliations to disclose;University of Florida Health:Salary/Stipend:Employment । Emily Plowman: Has affiliations to disclose;University of Florida:Salary/Stipend:Employment;Biohaven:Consulting fee:Consulting;ALS Association:Grant:Other Activities;National Institutes of Health:Grant:Other Activities. 
Relevant Non-financial Relationships: Lauren DiBiase: Has a NonFinancial Disclosure Affliation;American Speech-Language-Hearing Association:Professional:Membership | Justine Dallal York: Has a Non-Financial Disclosure Affliation;American Speech-LanguageHearing Association:Professional:Membership;Dysphagia Research Society:Professional:Membership | Kayla Croft: Nothing to Disclose I Tara Segalewitz: Has a Non-Financial Disclosure Affliation;American

Speech-Language-Hearing Association:Professional:Membership I Shelby Lawrence: Has a NonFinancial Disclosure Affliation;American Speech-Language-Hearing Association:Professional:Membership | Grace Vernon: Has a NonFinancial Disclosure Affliation;American Speech-Language-Hearing Association:Professional:Membership | Krista Boesing: Has a NonFinancial Disclosure Affliation;American Speech-Language-Hearing Association:Professional:Membership I Shauna Mackey: Has a NonFinancial Disclosure Affliation;American Speech-Language-Hearing Association:Professional:Membership I Kelly Leonard: Has a NonFinancial Disclosure Affliation;American Speech-Language-Hearing Association:Professional:Membership | Amber Anderson: Has a NonFinancial Disclosure Affliation;American Speech-Language-Hearing Association:Professional:Membership | Nicole Herndon: Has a NonFinancial Disclosure Affliation;American Speech-Language-Hearing Association:Professional:Membership | Emily Plowman: Has a NonFinancial Disclosure Affliation;American Speech-Language-Hearing Association:Professional:Membership;Dysphagia Research Society:Professional:Membership;Northeast ALS Consortium (NEALS):Professional:Volunteer membership on advisory committee or review panels.

\section{The Neuromuscular Development of Swallowing Continues into the School-Age Years: Evidence from a Preliminary Cross-sectional Study}

\section{Rachel Hahn Arkenberg ${ }^{1}$, Samantha Mitchell², Barb Brown', Lisa Goffman $^{3}$, Georgia Malandraki ${ }^{4}$}

Purpose: There is a gap in our knowledge of the typical development of the neuromuscular control of swallowing throughout childhood. To address this, we compared the neuromuscular control of swallowing in children at two distinct stages of neurodevelopment: 7 years old, when there is continuing cortical thickening, and 11-12 years old, a range that encompasses the peak of gray matter volume for children. Method(s): This was a prospective cross-sectional study. Data were collected from two groups of children aged 7 and 11-12 years. All children were screened for normal development (intelligence and language). Neuromuscular control was evaluated using surface electromyography (sEMG) of the submental muscles during swallowing of self-administered trials of $5 \mathrm{ml}$ water, $10 \mathrm{ml}$ water, cup sip water, sequential cup sip water, $5 \mathrm{cc}$ pudding, and bites of pretzel $(3 \times$ each). Surface EMG outcomes included normalized mean amplitude, bilateral synchrony, and burst duration of the smoothed sEMG signal. Non-parametric analyses (Wilcoxon signed-rank test) were utilized to analyze the data at this stage.

Result(s): Data from 13 children ( 5 male, 8 female; 6 younger, 7 older) have been collected and analyzed. The Wilcoxon signed-rank test revealed statistically significant differences between the age groups in normalized mean amplitude for both $5 \mathrm{~mL}$ and $10 \mathrm{~mL}$ thin liquid $(p<0.05)$. Younger children were observed to have higher mean amplitude values of submental muscle activity during all trials, though the difference in solid and pudding trials did not reach statistical significance. Across all tasks, younger children demonstrated more variability (e.g., submental muscle amplitude during $5 \mathrm{cc}$ pudding bolus, younger $\mathrm{SD}=6.42$, older $\mathrm{SD}=3.62$ )
Conclusions (Including Clinical Relevance): This preliminary study supports that typically developing children continue to demonstrate refinement of neuromuscular control of swallowing between 7 and 11-12 years of age. Although this is a preliminary (and ongoing) study, to our knowledge it is the first to document protracted neuromuscular development of swallowing in older children. Mapping the development of swallowing during later childhood may have significant implications for diagnosis and treatment of clinical populations in the future.

Relevant Financial Relationships: Rachel Hahn Arkenberg: Has affiliations to disclose;Purdue University:Salary/Stipend:Employment;Indiana Lions Speech and Hearing:Grant:Other Activities । Samantha Mitchell: Has affiliations to disclose;Purdue University:Salary/Stipend:Employment;NIH:Salary/Stipend:Other Activities I Barb Brown: Has affiliations to disclose;Purdue University:Salary/ Stipend:Employment;NIH:Salary/Stipend:Employment I Lisa Goffman: Has affiliations to disclose;NIH:Consulting fee:Consulting;University of Texas Dallas:Salary/Stipend:Employment । Georgia Malandraki: Has affiliations to disclose;Purdue University:Salary/Stipend:Employment;NIH:Grant:Other Activities.

Relevant Non-financial Relationships: Rachel Hahn Arkenberg: Has a Non-Financial Disclosure Affliation;American Speech Language Hearing Association:Professional:Membership;Dysphagia Research Society:Professional:Membership | Samantha Mitchell: Has a Non-Financial Disclosure Affliation;Dysphagia Research Society:Professional:Membership | Barb Brown: Has a Non-Financial Disclosure Affliation;American Speech Language Hearing Association:Professional:Membership I Lisa Goffman: Has a Non-Financial Disclosure Affliation;American Speech Language Hearing Association:Professional:Membership I Georgia Malandraki: Has a NonFinancial Disclosure Affliation;Dysphagia Research Society:Professional:Board membership;American Speech Language Hearing Association:Professional:Membership.

\section{Swallowing Function and Its Factors Influencing Oral Feeding in Patients with Persistent Disorder of Consciousness}

Yoko Inamoto $^{1}$, Shoko Kajiura ${ }^{2}$, Keiko Aihara', Seiko Shibata ${ }^{2}$, Marlis Gonzalez-Fernandez ${ }^{3}$, Eiichi Saitoh ${ }^{2}$

Purpose: Dysphagia is common for patients who experience disorders of consciousness (DOC) after severe acquired brain injury. However, there are no large studies investigating chronic swallowing dysfunction in this population. This study aimed to describe swallowing in patients with persistent DOC after severe acquired brain injury using videofluoroscopy (VF) and to understand the factors influencing oral feeding.

Method(s): Seventy-eight patients in persistent vegetative state (37 \pm 17 years old, average length of hospital stay $20 \pm 19$ months $)$ who were hospitalized between January 2001 and October 2018 for performing treatment of DOC using dorsal column stimulation (DCS) and for dysphagia rehabilitation were recruited. All patients were evaluated using VF after DCS. Using VF, we evaluated the following parameters: (1) bolus transport from the oral cavity to pharynx (active use of the tongue vs. gravity), (2) spontaneous swallowing (triggered without facilitation, triggered with facilitation, or no swallowing triggered), and (3) presence of aspiration and cough.

Result(s): Nine percent of patients actively transported the bolus to pharynx using the tongue and $91 \%$ by gravity. Swallowing was triggered without facilitation in $58 \%$ of patients, with facilitation in $39 \%$ of patients, and not triggered in $3 \%$ of patients. Thirty-six percent of patients had aspiration. Aspiration was silent in all cases. Fourty-seven patients $(60 \%)$ were able to start some oral feeding after 
VF using compensatory strategies such as posture and food modification. Of these 47 patients, 32 patients were tube fed before VF. Those who could start some oral feeding were more likely to have active bolus transport using the tongue, swallowing without facilitation, and less aspiration $(p<0.05)$.

Conclusions (Including Clinical Relevance): Bolus transport was mostly due to gravity and tongue propulsion was rare in patients with DOC. Our results indicate that evaluating the ability to trigger a swallow without facilitation and using VF to find the individualized intervention for avoiding aspiration are essential. VF is useful to evaluate risks and to identify the compensating strategies to allow for oral feeding in these patients.

Relevant Financial Relationships: Yoko Inamoto: Nothing to Disclose I Shoko Kajiura: Nothing to Disclose I Keiko Aihara: Nothing to Disclose I Seiko Shibata: Nothing to Disclose I Marlis GonzalezFernandez: Nothing to Disclose I Eiichi Saitoh: Nothing to Disclose. Relevant Non-financial Relationships: Yoko Inamoto: Nothing to Disclose I Shoko Kajiura: Nothing to Disclose I Keiko Aihara: Nothing to Disclose I Seiko Shibata: Nothing to Disclose I Marlis Gonzalez-Fernandez: Nothing to Disclose I Eiichi Saitoh: Nothing to Disclose.

\section{The Neuromuscular Control of Swallowing and Speech in Unilateral CP: Overactivation and Lack of Specificity are Overlapping Traits}

\section{Georgia Malandraki ${ }^{1,2}$, Samantha Mitchell ${ }^{1}$, Rachel Hahn Arkenberg ${ }^{1}$, Barbara Brown ${ }^{1}$, Jennifer Lundine ${ }^{3,4}$, Wendelin Burdo-Hartman $^{4,5}$, Bruce Craig ${ }^{6}$, Lisa Goffman ${ }^{7}$}

Purpose: Swallowing and speech share physiological substrates and are often impacted in cerebral palsy (CP), but have been traditionally treated as distinct functions. To start determining separate and common neuromuscular correlates of swallowing and speech in $\mathrm{CP}$, we completed a cross-sectional study in children with unilateral CP (UCP) and typically developing children (TDC).

Method(s): Sixteen children with UCP (10 male; age range: 7;2-12;2 yoa, GMFCS: I-II) and 16 age and sex-matched TDC participated. Children participated in a large-scale project including standardized swallowing, speech and language assessments, surface electromyography (sEMG), and an MRI scan. This abstract focuses on sEMG data. Surface EMG was recorded from the submental and the superior and inferior orbicularis oris muscles during homologous tasks, increasing in motor complexity, including: $5 \mathrm{ml}$ and $10 \mathrm{ml}$ water and $5 \mathrm{cc}$ pudding swallows; and 2-syllable words, 4-syllable words, and short sentences, all heavy in bilabial sounds. Outcome measures were normalized mean amplitude and zero-lag cross-correlations of bilateral muscle pairs. Differences in group means were assessed via nonparametric bootstrap.

Result(s): Children with UCP presented with consistently higher sEMG amplitude of all muscles across tasks compared to TDC. Amplitude was significantly higher in the UCP group on all muscle pairs for $10 \mathrm{ml}$ water (95\% CI 0.12-11.29 submental; 2.24-13.68 sup. orbicularis oris; $2.07-18.71$; inf. orbicularis oris), on the sup. orbicularis oris for $5 \mathrm{ml}$ water $(95 \%$ CI $0.82-10.17)$, on the inf. orbicularis oris-affected side for $5 \mathrm{ml}$ water and pudding (95\% CI 0.87-23.45), and for the submental pairs and the inf. orbicularis oris-affected side for speech (95\% CI 2.09-21.88 submental 2 syllable; 4.86-32.49 submental 4 syllable; $1.99-27.84$ inf. orbicularis oris-affected 2 syllable; and 8.12-48.95 inf. orbicularis oris 4 syllable). Also, the UCP group exhibited reduced bilateral synchrony of the submental muscles for the $5 \mathrm{ml}$ water and the $5 \mathrm{cc}$ pudding and of the sup. orbicularis oris for the 4-syllable task.
Conclusions (Including Clinical Relevance): These findings reveal that across swallowing and speech, children with UCP exhibit orofacial neuromuscular overactivation. This indicates that neural commands driving these functions are less specific in these children and provides insights on potential neuromuscular treatment targets.

Relevant Financial Relationships: Georgia Malandraki: Has affiliations to disclose;Purdue University:Salary/ Stipend:Employment;NIH:Grant:Other Activities;AACPDM:Grant:Other Activities;Purdue University:Grant:Other Activities I Samantha Mitchell: Has affiliations to disclose;Purdue University:Salary/Stipend:Employment;NIH:Salary/Stipend:Employment I Rachel Hahn Arkenberg: Has affiliations to disclose;Purdue University:Salary/Stipend:Employment | Barbara Brown: Has affiliations to disclose;Purdue University:Salary/ Stipend:Employment;NIH:Salary/Stipend:Employment | Jennifer Lundine: Has affiliations to disclose;Ohio State University:Salary/ Stipend:Employment;AACPDM:Grant:Other Activities I Wendelin Burdo-Hartman: Has affiliations to disclose;Nationwide Children's Hospital:Salary/Stipend:Employment;AACPDM:Grant:Other Activities I Bruce Craig: Has affiliations to disclose;Purdue University:Salary/Stipend:Employment;NIH:Grant:Other Activities I Lisa Goffman: Has affiliations to disclose;University of Texas, Dallas:Salary/Stipend:Employment;NIH:Consulting fee:Consulting.

Relevant Non-financial Relationships: Georgia Malandraki: Has a Non-Financial Disclosure Affliation;Dysphagia Research Society:Professional:Board membership;ASHA:Professional:Membership;ASHA Journals:Professional:Volunteer membership on advisory committee or review panels;VA Merit Grants Panel:Professional:Volunteer membership on advisory committee or review panels;Sheppard Dysphagia Institute:Professional:Vounteer consulting;AACPDM:Professional:Membership I Samantha Mitchell: Has a Non-Financial Disclosure Affliation;Dysphagia Research Society:Professional:Membership;NSSLHA:Professional:Membership | Rachel Hahn Arkenberg: Has a Non-Financial Disclosure Affliation;Dysphagia Research Society:Professional:Membership;ASHA:Professional:Membership | Barbara Brown: Has a Non-Financial Disclosure Affliation;ASHA:Professional:Membership | Jennifer Lundine: Has a NonFinancial Disclosure Affliation;ASHA:Professional:Membership I Wendelin Burdo-Hartman: Has a Non-Financial Disclosure Affliation;Dysphagia Research Society:Professional:Membership;AACPDM:Professional:Membership I Bruce Craig: Nothing to Disclose I Lisa Goffman: Has a NonFinancial Disclosure Affliation;ASHA:Professional:Membership.

\section{An exploration of Clinical Reasoning in Dysphagia Rehabilitation}

\section{Thiani Pillay $^{1}$, Mershen Pillay ${ }^{1}$}

Purpose: Historically, dysphagia rehabilitation has been considered from a largely positivistic and biomedical standpoint. This approach is paramount to guide the management of the impairment-based condition. However, its prescriptive nature often results in the heterogeneous narratives and understandings of clinicians and patients being dismissed. This standpoint is unrealistic in the clinical encounter given the personal nature of eating. This study aimed to challenge this understanding by exploring clinical reasoning from an intersectional standpoint. This was achieved by focusing on the relationship between a clinician's identity and their approach to clinical reasoning within dysphagia rehabilitation. By exploring this nuanced relationship between clinical reasoning and identity, we are able to advocate for a more inclusive approach to dysphagia assessment which shows the value of heterogeneity. 
Method(s): Exploratory interviews were undertaken with seven South African-trained SLPs with experience working in dysphagia. Three alternative qualitative techniques were employed within the interviews namely oral histories, cognitive mapping, and PhotoVoice. An initial thematic analysis followed by a further intersectional ideological analysis was undertaken.

Result(s): The results of the study revealed four core themes with corresponding photographs which showed the impact of knowledge, context, identity and colonization in the use and development of clinical reasoning amongst speech-language therapists working South Africa.

Conclusions (Including Clinical Relevance): By considering clinical reasoning from a different epistemological standpoint, we begin to rethink dysphagia to account for a more inclusive approach which values the input of complexity. This has significant implications for future practice particularly within an Afrocentric context given the diverse and complex nature of our socioeconomic circumstances.

Relevant Financial Relationships: Thiani Pillay: Nothing to Disclose I Mershen Pillay: Has affiliations to disclose;University of KwaZulu-Natal:Salary/Stipend:Employment.

Relevant Non-financial Relationships: Thiani Pillay: Nothing to Disclose I Mershen Pillay: Nothing to Disclose.

\section{Prevalence and Demographics of Dysphagia in COVID- 19-Hospitalized Patients}

\section{Courtney Tipton ${ }^{1}$}

Purpose: The purpose of this study was to better understand the rates, potential risk factors, and complications of dysphagia in patients hospitalized with COVID-19. The overall goal was to better understand what patient populations may be at greatest risk and necessitate early intervention for dysphagia.

Method(s): A retrospective review was conducted of patients hospitalized with COVID-19 across five hospitals affiliated with our Institution from 03/01/2020 to 09/30/2020. Inclusion criteria were age $\geq 18$ and diagnosis of new onset dysphagia during the current admission by a speech-language pathologist (SLP). Exclusion criteria were premorbid diagnosis of dysphagia.

Result(s): 1009 unique medical record numbers were screened. 74 (7.3\%) patients had new-onset dysphagia diagnosed during their COVID-19 admission. 37 patients (50\%) were male and $37(50 \%)$ were female with an average age of 65 years (range 27-99). 42 $(56.8 \%)$ were African American/Black and 23 (31.1\%) White/Caucasian. Common co-morbidities included hypertension (70.2\%), diabetes mellitus $(45.9 \%)$, and a history of stroke prior to admission $(31.1 \%)$. On average, the first SLP evaluation occurred 11.4 days after admission. Only 15 people $(20.3 \%)$ completed a modified barium swallow study due to COVID-19 restriction protocols. Only 1 patient (1.4\%) had a fiberoptic endoscopic evaluation of swallowing, performed after discharge. Only $20(27.0 \%)$ patients resumed normal diet at discharge and $15(20.3 \%)$ required new percutaneous gastric tube insertion. $38(51.4 \%)$ were intubated for an average length of 11.9 days. The average length of stay was 22.9 days with an average of 4.8 consulting services for the admission. The 30-day mortality rate was $18.9 \%$ (14 patients). These data are being compared to COVID19 patients without dysphagia during this time period $(N=771)$.

Conclusions (Including Clinical Relevance): Although many providers focus on the cardiopulmonary effects of COVID-19, dysphagia can significantly complicate the patient's recovery. Early screening and intervention for patients with COVID-19 should be completed to prevent potential sequelae of dysphagia, including the need for longterm enteral access, increased length of stay, and mortality.
Relevant Financial Relationships: Courtney Tipton: Nothing to Disclose.

Relevant Non-financial Relationships: Courtney Tipton: Nothing to Disclose.

\section{Is There a Recency Effect During Visuoperceptual FEES Analysis?}

\section{$\underline{\text { James C. Borders }}^{1}$, James A. Curtis ${ }^{1}$, Michelle S. Troche ${ }^{1}$}

Purpose: Visuoperceptual ratings of the amount of airway invasion on FEES are common practice in research and clinical care. However, it is unknown whether this visuoperceptual analysis is influenced by the severity of prior swallows, and thus, if raters are prone to a recency bias. This study aimed to examine whether exposure to a prior swallow (either mild or severe) affected laryngeal vestibule ratings for the next swallow.

Method(s): Eight FEES-trained speech-language pathology master's students rated the amount of penetrant residue within the laryngeal vestibule using a 100-point visual analog scale (VAS). Ratings consisted of two blocks of 20 FEES video clips, including (1) "clustered" mild-only swallows $\left(M_{\mathrm{VAS}}=11.40\right)$ and $(2)$ "clustered" severe-only swallows $\left(M_{\mathrm{VAS}}=62.25\right)$, as well an additional third block of 40 video clips of "alternating" mild and severe swallows, which were replicated from the clustered blocks. Each block of video clips was rated one week apart, and the order of the blocks was randomized across raters. Linear mixed models and paired equivalence tests were used to determine if VAS ratings for mild and severe swallows were the same or different when rated as clustered or alternating blocks.

Result(s): Average VAS ratings for mild clustered swallows were $6.18(\mathrm{SD}=4.99)$ and mild alternating swallows were $7.49(\mathrm{SD}=$ $6.34)$, whereas severe clustered was $27.10(\mathrm{SD}=19.90)$ and severe alternating swallows were $28.20(\mathrm{SD}=19.80)$. There was no significant recency effect of exposure to a severe $(p=0.72)$ or mild $(p=0.96)$ swallow on subsequent laryngeal vestibule ratings. Both severe $(p<0.001)$ and mild $(p=0.034)$ clustered ratings were statistically equivalent to their alternating counterpart, providing evidence for the null hypothesis of no difference.

Conclusions (Including Clinical Relevance): Visuoperceptual analyses of laryngeal vestibule residue were not affected by prior mild or severe swallows, suggesting that trained raters are not prone to a recency effect when rating laryngeal vestibular residue with a visual analog scale. These findings support recent research on objective fluoroscopy analyses which have demonstrated that randomization of boluses may not be necessary to reduce rater error. Future research should explore the recency bias with expert clinicians, in the context of other anatomic landmarks, and between more subtle differences in rating severities (e.g., mild and moderate).

Relevant Financial Relationships: James Borders: Nothing to Disclose I James Curtis: Nothing to Disclose I Michelle Troche: Has affiliations to disclose;Dysphagia Research Society:Honoraria:Other Activities;MedBridge Inc:Royalty:Other Activities; Teachers College, Columbia University:Salary/Stipend:Employment;Michael J Fox Foundation:Grant:Independent contractor (Including contracted research);National Ataxia Foundation:Grant:Independent contractor (Including contracted research).

Relevant Non-financial Relationships: James Borders: Has a NonFinancial Disclosure Affliation;Dysphagia Research Society:Professional:Membership | James Curtis: Has a Non-Financial Disclosure Affliation;Dysphagia Research Society:Professional:Membership I Michelle Troche: Has a Non-Financial Disclosure Affliation;Dysphagia Research Society:Professional:Membership. 
Impact of Transoral Robotic Surgery vs. Radiation on Swallowing Function in Oropharyngeal Cancer Patients: A Sub-study from a Randomized Trial

Julie Theurer $^{2,4,6}$, David A. Palma ${ }^{1,2,4}$, Rosemary Martino ${ }^{3}$, John De Almeida $^{5}$, Kevin Fung ${ }^{1,2}$, John Yoo ${ }^{1,2}$, S. D. MacNeil ${ }^{1,2}$, Eric Winquist $^{1,2}$, Colleen Dreyer ${ }^{1}$, Sarah Hawkins ${ }^{1}$, Kendra Thouless ${ }^{1}$, Anthony C. Nichols ${ }^{1,2}$

Purpose: Attempts to reduce toxicities and increase quality of life (QOL) in patients treated for oropharyngeal cancer (OPC) have included transoral robotic surgery (TORS) as an alternative to radiation therapy (RT). The ORATOR trial (NCT01590355) provided the first randomized comparison of swallowing QOL following primary RT vs. primary TORS, with reported results based on patient questionnaires only. This sub-study of ORATOR compares physiologic swallowing outcomes between patients treated with RT vs. TORS. Method(s): Patients with early-stage OPC enrolled in ORATOR between July 2014 and February 2017 were eligible for additional swallowing evaluation. Modified barium swallow studies were administered at baseline, and 6- and 12-months post-treatment, and swallow function was analyzed using the Modified Barium Swallow Impairment Profile (MBSImP $\odot^{\mathrm{TM}}$ ) and Penetration-aspiration Scale (PAS). Differences between treatment arms were compared using parametric and nonparametric statistics and linear mixed modeling, as appropriate. Correlation between MBSImP scores and MD Anderson Dysphagia Inventory (MDADI) data and PAS across all time points were evaluated using Pearson correlation coefficients (PCC).

Result(s): Fifteen patients in ORATOR Arm 1 (RT \pm chemotherapy) and six patients in Arm 2 (TORS) (mean [ \pm SD] age, $56.3[ \pm 8.4]$ years) participated in this sub-study. Mean $[ \pm$ SD] MBSImP oral total scores for Arm 1 and Arm 2 patients were $4.8[ \pm 2.1]$ vs. $4.3[ \pm 1.5]$ at baseline, $5.4[ \pm 2.1]$ vs. $4.4[ \pm 2.5]$ at 6 months, and $4.6[ \pm 2.0]$ vs. $4.8[ \pm 2.1]$ at 12 months, respectively. Pharyngeal total scores for Arm 1 and Arm 2 patients at these timepoints were $5.6[ \pm 2.2]$ vs. 4.7 $[ \pm 2.2], 6.2[ \pm 1.2]$ vs. $9.6[ \pm 4.8]$, and $5.9[ \pm 1.8]$ vs. $8.0[ \pm 4.7]$, respectively. Highest PAS scores were observed at 6 months posttreatment. MBSImP oral total scores were not significantly correlated with any MDADI scale scores or PAS scores, while MBSImP pharyngeal total scores demonstrated weak correlations with MDADI composite ( $\mathrm{PCC}=-0.257, p=0.049)$, emotional $(\mathrm{PCC}=-0.283$, $p=0.028)$, and physical ( $\mathrm{PCC}=-0.288, p=0.027)$ subscales and PAS scores $(\mathrm{PCC}=0.374, p=0.003)$.

Conclusions (Including Clinical Relevance): Although likely underpowered to capture group differences, examination of physiologic swallowing function after RT vs. TORS for early-stage OPC revealed subtle non-significant differences that correspond to altered swallowing-related QOL as reported in the ORATOR trial.

Relevant Financial Relationships: Julie Theurer: Has affiliations to disclose;Western University:Salary/Stipend:Employment I David Palma: Nothing to Disclose I Rosemary Martino: Nothing to Disclose | John De Almeida: Nothing to Disclose | Kevin Fung: Nothing to Disclose I John Yoo: Nothing to Disclose I S. MacNeil: Nothing to Disclose | Eric Winquist: Nothing to Disclose | Colleen Dreyer: Nothing to Disclose I Sarah Hawkins: Nothing to Disclose I Kendra Thouless: Nothing to Disclose | Anthony Nichols: Nothing to Disclose.

Relevant Non-financial Relationships: Julie Theurer: Has a NonFinancial Disclosure Affliation;DRS:Professional:Membership । David Palma: Nothing to Disclose I Rosemary Martino: Has a NonFinancial Disclosure Affliation;DRS:Professional:Membership | John De Almeida: Nothing to Disclose I Kevin Fung: Nothing to Disclose I John Yoo: Nothing to Disclose I S. MacNeil: Nothing to Disclose I Eric Winquist: Nothing to Disclose | Colleen Dreyer: Nothing to
Disclose | Sarah Hawkins: Nothing to Disclose | Kendra Thouless: Nothing to Disclose I Anthony Nichols: Nothing to Disclose.

\section{Swallowing Symptoms Increase the Risk of Dynapenia in Community-Dwelling Oldest-Old: Retrospective Cohort Study}

\author{
Natalia Conessa Ortega ${ }^{1}$, Cinthia M. Souza ${ }^{1}$, Lucia Mourão ${ }^{1}$
}

Purpose: The aim of the study was to verify if swallowing symptoms, at baseline, may be risk factors for developing dynapenia, after 8 years, in community-dwelling oldest-old adults.

Method(s): It is a retrospective cohort study, based on data from an epidemiologic study on frailty in Brazilian elderly residents in the community, called FIBRA, conducted in two periods: 2008-2009 baseline (T0) and 2016-2017 follow-up (T1). Nutritional status was assessed by body mass index (BMI). In T0, it was evaluated selfreported questions related to swallowing symptoms: dry mouth in past 4 weeks, taste changes, chewing difficulties, swallowing difficulties, sensation of food stuck in throat, reflux of food to mouth and nose, clear throat after eat, choking after eating and drinking, and swallowing liquids after eating food. In the T1, the maximum handgrip strength (HGmax) was performed. The analysis of the principal components analysis (PCA) was performed with the self-reported questions. Univariate and multivariate logistic regression analysis was used with statistical significance $p<0.05$.

Result(s): It was included elderly with no cognitive impairment: 291 women $(69.5 \%)$ and $53.5 \%$ between 70 and 79 years $(53.5 \%)$. Swallowing symptoms were observed in 356 elderly. Dry mouth was reported by $44.7 \%$, chewing difficulties $34.6 \%$, and $6.1 \%$ swallowing difficulties. The PCA identified as component 1: swallowing difficulty, sensation of food stuck in throat, taste changes and chewing difficulties. Among BMI, in the T0, 14.7\% elderly were classified as underweight and, $17.5 \%$ in the $\mathrm{T} 1$, no difference was revealed in the comparison between $\mathrm{T} 0$ and $\mathrm{T} 1$. Univariate logistic regression showed that risk of dynapenia was 2.1 times higher in elderly with underweight ( $p=0.024$, OR 2.13, CI 1.11-4.11) and 3 times higher with swallowing symptoms ( $p=0.012$, OR 3.08.CI 1.28-7.44). In the multivariate regression, elderly males have 3 times higher risk ( $p=0.004$, OR 2.03, CI 1.26-3.28) and the component 1 of PCA showed 0.7 times ( $p=0.003$, [LM1] OR 0.710; CI 0.568-0.888) more risk to dynapenia.

Conclusions (Including Clinical Relevance): The presence of swallowing difficulty, sensation of food stuck in throat, taste changes, and chewing difficulties in the elderly males increase the risk of developing dynapenia after 8 years. The results reinforce the importance of preventive program related to swallowing and nutritional intervention to minimize the effects of dynapenia in the elderly.

Relevant Financial Relationships: Natalia Conessa Ortega: Nothing to Disclose I Cinthia Souza: Nothing to Disclose | Lucia Mourão: Nothing to Disclose.

Relevant Non-financial Relationships: Natalia Conessa Ortega: Nothing to Disclose I Cinthia Souza: Nothing to Disclose I Lucia Mourão: Nothing to Disclose.

\section{Within-Subject Changes to Swallowing Metrics After Anterior Cervical Discectomy and Fusion Surgery}

Sonja M. Molfenter ${ }^{1}$, Nicole Carambot ${ }^{2}$, Anthony FrempongBoadu $^{2}$, Matina Balou ${ }^{3}$ 
Purpose: The reported incidence of dysphagia post-ACDF varies widely (6.5 to $71 \%$ ) which is in part attributable to differences and inadequacies in the methods for capturing dysphagia. Many studies employ non-validated questionnaires about swallowing, fail to capture pre-surgical swallowing function, and/or do not standardize the time post-surgery for measurement. The purpose of this study was to prospectively establish within-subject changes to swallowing function as the result of ACDF at our institution using validated patient-reported outcome (PRO) and diet scale metrics.

Method(s): 35 patients ( 23 male, mean age $=52.7, \mathrm{SD}=10.4$ ) were prospectively enrolled in this study. The most common surgical levels included C5-C7 (16/35), C4-C6 (8/35), and C4-C7 (5/35). Patients completed EAT-10 and SWAL-QOL questionnaires pre-ACDF and 4 weeks post-ACDF. Functional Oral Intake Scale (FOIS) scores were collected pre-ACDF and on discharge from the hospital. McNemar Chi Square tests examined the change in distribution of abnormal EAT-10 scores ( $>3$ ) pre- vs post-ACDF. Paired t tests compared SWAL-QOL scores (overall and individual domains) prevs post-ACDF. Wicoxon Signed Rank Test examined the change in distribution of FOIS scores pre-ACDF to discharge. $p<0.01$ was considered significant to control for multiple comparisons.

Result(s): Median EAT-10 scores increased from 0 pre-ACDF to 7 post-ACDF. Significantly more patients had abnormal $(>3)$ EAT-10 scores post-ACDF $(24 / 35=69 \%)$, compared with pre-ACDF $(6 /$ $35=17 \%)[p>0.001]$. Total SWAL-QOL scores were significantly worse post-ACDF (mean $=83.6, \mathrm{SD}=12.8)$ compared to pre-ACDF (mean $=93.1, \mathrm{SD}=5.8)[t=4.7, p<0.001]$. Specific domains with significant impairment included: burden $(t=4.9, p<0.001)$, eating duration $(t=3.6, \quad p=0.001)$, symptom frequency $(t=4.4$, $p<0.001)$, food selection $(t=3.5, p=0.001)$, and fear $(t=4.0$, $p<0.001)$. FOIS scores were significantly reduced at discharge (median $=6)$ compared with pre-ACDF $($ median $=7)[Z=-4.1$, $p<0.001]$.

Conclusions (Including Clinical Relevance): This prospective, within-subject analysis using validated swallowing PROs and diet scales confirms that a large proportion of patient experience a significant disruption to swallowing function and quality of life postACDF. This work will set the stage for future investigations into the pathophysiology of dysphagia post-ACDF with gold-standard imaging in collaboration with the neurosurgery team at our institution.

Relevant Financial Relationships: Sonja Molfenter: Has affiliations to disclose;NYU:Salary/Stipend:Employment I Nicole Carambot: Nothing to Disclose I Anthony Frempong-Boadu: Nothing to Disclose I Matina Balou: Has affiliations to disclose;NYU Langone Health and NYU Grossman School of Medicine:Salary/Stipend:Employment.

Relevant Non-financial Relationships: Sonja Molfenter: Has a NonFinancial Disclosure Affliation;DRS:Professional: Other volunteer activities I Nicole Carambot: Nothing to Disclose I Anthony Frempong-Boadu: Nothing to Disclose I Matina Balou: Has a NonFinancial Disclosure Affliation;DRS:Professional: Other volunteer activities;NYSSLHA:Professional:Board membership.

\section{Use of Diffusion Tensor Imaging to Examine Microstructural Changes Following Lingual Strengthening Exercise}

\section{Brittany N. Krekeler ${ }^{1,2}$, Hou Jiancheng ${ }^{3}$, Veena Nair ${ }^{3,4}$, Vivek Prabhakaran $^{3}$, Nicole Rogus-Pulia ${ }^{2,5,6}$, JoAnne Robbins ${ }^{5}$}

Purpose: Central effects of lingual strengthening exercise (LSE) as a treatment for dysphagia remain largely unknown. Diffusion tensor imaging (DTI) is a neuroimaging approach used to identify differences in microstructural changes in white matter tracts driving neural connectivity in the brain. Alterations in white matter structure resulting from clinical interventions can indicate central changes to neural signal processing. The purpose of this pilot study was to measure microstructural white matter changes following LSE in a group of patients with dysphagia.

Method(s): Diffusion-weighted images were acquired from 7 participants with dysphagia on videofluoroscopy (avg age $=70$; various medical conditions) before and after a lingual exercise protocol (20 reps, $3 \mathrm{x} /$ day, 3 days/week, 8 weeks) using a 10-min diffusion sequence, 9 b0, 56 directions (with b1000) on GE750 3T scanner. Tract-Based Spatial Statistics were used to evaluate voxel-based group differences for values of fractional anisotropy, mean diffusivity (MD), axial diffusivity (AD), radial diffusivity (RD), and local diffusion homogeneity (LDH). Paired $t$ tests were performed to investigate the difference between pre-treatment and post-treatment on each DTI metric $(p<0.05)$ using IBM SPSS v23.

Result(s): Maximum pressure generation at the front $(\mathrm{avg}=46.41)$ and back (104.33) lingual sensors increased after exercise. MD and $\mathrm{RD}$ in right inferior fronto-occipital fasciculus (IFOF) were increased, as well as increased LDH in the bilateral anterior thalamic radiation (ATR). However, decreases in AD in the bilateral IFOF, MD in the right uncinate fasciculus (UF), and $\mathrm{AD}$ in the left UF were also observed. No significant changes in fractional anisotropy were noted. Conclusions (Including Clinical Relevance): Findings of increased $\mathrm{MD}$ and $\mathrm{RD}$ in the right IFOF may indicate improvement in connectivity between language processing and goal-oriented behavior; while decreases in MD and AD in the UF may point to alterations in episodic memory. Increased LDH in bilateral ATR regions may indicate improved relay of somatosensory information to the cortex. These microstructural changes observed via DTI in this heterogeneous group of patients with dysphagia suggest that LSE may alter critical central neural pathways related to learning and sensorimotor relay. Future investigations should explore these effects in larger groups of homogeneous patients.

Relevant Financial Relationships: Brittany Krekeler: Has affiliations to disclose;Northwestern Univeristy:Salary/ Stipend:Employment I Hou Jiancheng: Has affiliations to disclose;UW-Madison:Salary/Stipend:Employment I Veena Nair: Has affiliations to disclose;UW-Madison:Salary/Stipend:Employment । Vivek Prabhakaran: Has affiliations to disclose;UW-Madison:Salary/ Stipend:Employment I Nicole Rogus-Pulia: Has affiliations to disclose;UW-Madison:Salary/Stipend:Employment I JoAnne Robbins: Has affiliations to disclose;Swallow Solutions:Ownership interest(e.g., stocks, stock options or other ownership interest excluding diversified mutual funds):Ownership.

Relevant Non-financial Relationships: Brittany Krekeler: Has a Non-Financial Disclosure Affliation;DRS:Professional:Membership I Hou Jiancheng: Nothing to Disclose I Veena Nair: Nothing to Disclose I Vivek Prabhakaran: Nothing to Disclose I Nicole Rogus-Pulia: Has a Non-Financial Disclosure Affliation;DRS:Professional:Membership | JoAnne Robbins: Has a Non-Financial Disclosure Affliation;DRS:Professional:Membership.

\section{Is There Evidence of Tongue Fatigue in People with Neurodegenerative Disease After Completion of a 21-Item Swallowing Assessment?}

\section{Pooja Gandhi $^{1,2}$, Emily Plowman ${ }^{3}$, Catriona M. Steele ${ }^{1,4}$}

Purpose: Previous studies have suggested that the act of eating a meal may induce fatigue in healthy older adults. Bulbar muscle weakness is recognized to be a component of dysphagia in people with Amyotrophic Lateral Sclerosis (pwALS) and Parkinson Disease (pwPD). However, little is known about tongue fatigability in these populations. Therefore, we aimed to measure tongue fatigability in 
pwALS and pwPD by comparing lingual pressure generation capacity before and after completing a videofluoroscopy (VFSS). We hypothesized that both patient cohorts would demonstrate post-VFSS reductions in lingual pressure generation capacity.

Method(s): Participants included 17 pwALS (mean age 62.5) and 20 pwPD (mean age 67) who completed a research VFSS involving 21 boluses of $20 \% \mathrm{w} / \mathrm{v}$ barium: 3 boluses each of thin; xanthan-gumthickened slightly thick, mildly thick, moderately thick, and extremely thick liquid; and starch-thickened slightly and moderately thick liquid. Baseline and post-VFSS measures of tongue strength on maximum isometric tasks and saliva swallows were collected using the Iowa Oral Performance Instrument. Outcomes of interest included pre-post VFSS change in maximum anterior isometric pressures (MAIP); maximum posterior anterior isometric pressures (MPIP); regular effort saliva swallows (RESS); and lingual functional reserve (LFR, defined as MAIP-RESS). Between groups analyses of variance were performed with factors of cohort (ALS, PD) and sex, with a $p$ value of 0.05 .

Result(s): Mean pre-post VFSS changes were small for all measures. There were no significant effects of sex or cohort on pre-post changes in MAIP and MPIP, which averaged - $1 \mathrm{kPa}$ overall (95\% confidence interval -4 to $+1 \mathrm{kPa})$. A significant cohort difference $(p=0.03)$ was found for changes in RESS, with a mean change of $-3 \mathrm{kPa}$ in the pwPD $(95 \% \mathrm{CI}-5$ to 0$)$ versus $+1 \mathrm{kPa}(95 \% \mathrm{CI}-1$ to +4$)$ in the pwALS. No significant differences were found in LFR, with an average pre-post change of $+1 \mathrm{kPa}(95 \% \mathrm{CI}-2$ to +3$)$.

Conclusions (Including Clinical Relevance): Despite having neurodegenerative diagnoses, the participants in this study showed no evidence of lingual fatigue after participating in a research videofluoroscopy. Additional research is needed to identify protocols that reliably reveal tongue or swallowing-related fatigue in healthy adults so that the impact of neurodegenerative disease on fatigability can be better understood.

Relevant Financial Relationships: Pooja Gandhi: Has affiliations to disclose;KITE-Toronto Rehabilitation Institute, University Health Network:Scholarship:Other Activities;KITE—-Toronto Rehabilitation Institute, University Health Network:Salary/Stipend:Employment;Rehabilitation Sciences Department, University of Toronto:Scholarship:Other Activities I Emily Plowman: Has affiliations to disclose;University of Florida:Salary/ Stipend:Employment;National Institute of Deafness and Other Communication Disorders:Grant:Independent contractor (Including contracted research) I Catriona Steele: Has affiliations to disclose;KITE-Toronto Rehabilitation Institute, University Health Network:Salary/Stipend:Employment;National Institute of Deafness and Other Communication Disorders:Grant:Independent contractor (Including contracted research).

Relevant Non-financial Relationships: Pooja Gandhi: Nothing to Disclose I Emily Plowman: Has a Non-Financial Disclosure Affliation;Dysphagia Research Society:Professional:Membership I Catriona Steele: Has a Non-Financial Disclosure Affliation;Dysphagia Research Society:Professional:Membership;Journal of Texture Studies:Professional:Board membership;International Dysphagia Diet Standardization Initiative:Professional:Membership;Dysphagia Journal:Professional:Board membership.

\section{Distinct Surgical and Endotracheal Risk Factors are Associated with Aspiration and Vocal Fold Paralysis in Cardiovascular Surgery Patients}

Emily K. Plowman ${ }^{1,2}$, Amber Anderson 2,3, Justine Dallal York ${ }^{2,3}$, Lauren DiBiase $^{2,3}$, Julia Eckart Iszler ${ }^{2,4}$, Shelby Lawrence ${ }^{2,4}$, Eric Jeng $^{5}$, Neil Chheda ${ }^{6}$
Purpose: Risk factors for aspiration and vocal fold mobility impairment (VFMI) in cardiovascular surgical patients are currently unclear. This knowledge gap has hindered implementation of triaged postoperative care pathways and development of preventative best practices guidelines. We, therefore, examined risk factors associated with aspiration and VFMI in postoperative cardiovascular patients.

Method(s): A prospective single-center study was conducted in postoperative adult cardiovascular patients between February 2019 and January 2020. Enrolled patients underwent a fiberoptic endoscopic evaluation of swallowing within $72 \mathrm{~h}$ of extubation. Demographic, surgical, and intubation outcomes were collected. Blinded ratings of the penetration aspiration scale (PAS) and vocal fold mobility impairment were performed. Univariate and multivariable regression analyses were completed with odds ratios (OR) and 95\% confidence intervals $(\mathrm{CI})$.

Result(s): 200 individuals participated in this study. Aspiration (PAS $\geq 6$ ) was confirmed in $28 \%$ of patients. Independent aspiration risk factors included New York Heart Association Score $\geq 3$ (OR 2.9), reoperation (OR 2.0), intubation $>27 \mathrm{~h}$ (OR 2.1), and endotracheal tube (ETT) size $\geq 8.0$ (OR 2.9). VFMI was confirmed in $23 \%$ of patients ( 7 complete, 39 partial VFMI), with left-sided involvement in $83 \%$ of cases. Independent VFMI risk factors for complete VFMI were aortic arch surgical procedure (OR 6.1), body mass index $<25.0$ (OR 7.2), and African American or Hispanic race (OR 6.0).

Conclusions (Including Clinical Relevance): No common risk factors were identified suggesting distinct underlying mechanisms for aspiration and VFMI in cardiovascular surgical patients. Left-sided laterality and VFMI associations with arch-related surgeries suggest that local and direct trauma and/or intraoperative mobilization and traction injury to the left RLN at the level of the aortic arch are the primary contributing processes of VFMI. In contrast, the identified endotracheal risk factors for aspiration are suggestive of mechanical damage to the upper aerodigestive tract and compression injury to the terminal branches of the RLN in the larynx from prolonged intubations and oversized ETTs. Future research utilizing preoperative imaging and intraoperative RLN monitoring techniques are needed to confirm these preliminary findings.

Relevant Financial Relationships: Emily Plowman: Has affiliations to disclose;University of Florida:Salary/Stipend:Employment;Biohaven:Consulting fee:Consulting;ALS Association:Grant:Other Activities;National Institutes of Health:Grant:Other Activities । Amber Anderson: Has affiliations to disclose;University of Florida:Salary/Stipend:Employment I Justine Dallal York: Has affiliations to disclose;University of Florida:Salary/Stipend:Employment I Lauren DiBiase: Has affiliations to disclose;University of Florida:Salary/ Stipend:Employment | Julia Eckart Iszler: Has affiliations to disclose;University of Florida:Salary/Stipend:Employment I Shelby Lawrence: Has affiliations to disclose;University of Florida:Salary/ Stipend:Employment I Eric Jeng: Has affiliations to disclose;University of Florida:Salary/Stipend:Employment;University of Florida Department of Surgery New Investigator Award:Grant:Other Activities I Neil Chheda: Has affiliations to disclose;University of Florida:Salary/Stipend:Employment.

Relevant Non-financial Relationships: Emily Plowman: Has a NonFinancial Disclosure Affliation;Dysphagia Research Society:Professional:Membership;American Speech-Language-Hearing Association:Professional:Membership;Northeast ALS Consortium (NEALS):Professional:Volunteer membership on advisory committee or review panels I Amber Anderson: Has a Non-Financial Disclosure Affliation;American Speech-Language-Hearing Association:Professional:Membership | Justine Dallal York: Has a Non-Financial Disclosure Affliation;Dysphagia Research Society:Professional:Membership;American Speech-Language-Hearing Association:Professional:Membership | Lauren DiBiase: Has a NonFinancial Disclosure Affliation;American Speech-Language-Hearing 
Association:Professional:Membership | Julia Eckart Iszler: Has a Non-Financial Disclosure Affliation;American Speech-LanguageHearing Association:Professional:Membership I Shelby Lawrence: Has a Non-Financial Disclosure Affliation;American Speech-Language-Hearing Association:Professional:Membership | Eric Jeng: Nothing to Disclose I Neil Chheda: Nothing to Disclose.

\section{Comparison of Lingual Pressure Generation Capacity in Amyotrophic Lateral Sclerosis, Parkinson Disease and Healthy Aging}

\section{Pooja Gandhi ${ }^{1,3}$, Emily K. Plowman ${ }^{2}$, Catriona M. Steele ${ }^{1,3}$}

Purpose: The tongue plays a key role in bolus propulsion in swallowing, with reduced tongue pressures thought to represent a risk for impaired swallowing safety and efficiency. Measures of maximum anterior isometric pressure ("MAIP") and regular effort saliva swallows ("RESS") can be used to quantify tongue strength and lingual functional reserve ("LFR", i.e., MAIP-RESS). We aimed to profile and compare lingual pressure generation capacity in patients with Amyotrophic Lateral Sclerosis (ALS), Parkinson Disease (PD) and in healthy adults over the age of 60 . We hypothesized that both patient cohorts would demonstrate reduced MAIP and RESS compared to the healthy control group, with the greatest reductions expected in the ALS cohort.

Method(s): Data were collected in 17 people with ALS (pwALS) (mean age 62.5), 20 people with PD (pwPD) (mean age 67) and in 20 healthy controls (mean age 70). MAIP and RESS measures were collected in $\mathrm{kPa}$ using the Iowa Oral Performance Instrument, in the anterior position. Three repetitions of each task were collected with rest between trials. Outcomes of interest included mean MAIP, mean RESS, and mean LFR. Between groups univariate analyses of variance were performed, with alpha set at 0.05 , and post-hoc Sidak tests of pairwise comparisons.

Result(s): Mean MAIP values were significantly lower $(p<0.01)$ in pwALS (34 kPa) than in healthy controls $(47 \mathrm{kPa})$ and pwPD $(55 \mathrm{kPa})$. MAIP values in pwPD showed a non-significant trend to being higher than those in healthy adults. RESS values did not differ significantly across groups (pwALS: $19 \mathrm{kPa}$, Healthy: $24 \mathrm{kPa}$; pwPD: $17 \mathrm{kPa})$. LFR was significantly higher $(p<0.01)$ in pwPD $(37 \mathrm{kPa})$ versus healthy control $(24 \mathrm{kPa})$ and pwALS $(15 \mathrm{kPa})$.

Conclusions (Including Clinical Relevance): In this study, lingual pressure generation capacity was reduced in pwALS, but not in pwPD, beyond reductions expected with healthy aging. Both patient cohorts displayed preservation lingual pressure during saliva swallows. Future studies exploring longitudinal changes in tongue pressure generation on isometric and saliva swallowing tasks will be needed to determine whether tongue pressure measures serve as biomarkers of swallowing impairment.

Relevant Financial Relationships: Pooja Gandhi: Has affiliations to disclose;KITE-Toronto Rehabilitation Institute, University Health Network:Scholarship:Other Activities;KITE—-Toronto Rehabilitation Institute, University Health Network:Salary/Stipend:Employment;Rehabilitation Sciences Department, University of Toronto:Scholarship:Other Activities I Emily Plowman: Has affiliations to disclose;University of Florida:Salary/ Stipend:Employment;National Institute of Deafness and Other Communication Disorders:Grant:Independent contractor (Including contracted research) I Catriona Steele: Has affiliations to disclose; NNational Institute of Deafness and Other Communication Disorders:Grant:Independent contractor (Including contracted research);KITE - Toronto Rehabilitation Institute, University Health Network:Salary/Stipend:Employment.
Relevant Non-financial Relationships: Pooja Gandhi: Nothing to Disclose I Emily Plowman: Has a Non-Financial Disclosure Affliation;Dysphagia Research Society:Professional:Membership | Catriona Steele: Has a Non-Financial Disclosure Affliation;Dysphagia Research Society:Professional:Membership;Journal of Texture Studies:Professional:Board membership;International Dysphagia Diet Standardization Initiative:Professional:Membership;Dysphagia Journal:Professional:Board membership.

\section{Differences in Thickened Formula Attributes: Influence of Cereal Type and Cereal Pulverization}

\author{
Abbey Sterkowitz ${ }^{3}$, Abigail M. Spoden ${ }^{3}$, Harrison Frisk ${ }^{1}$, Michael \\ Beckstrand $^{2}$, Katlyn E. McGrattan ${ }^{3}$
}

Purpose: Background: Infant rice and oatmeal cereals are commonly used thickening agents among young infants. Difficulties in fully dissolving these cereals in infant formula often lead clinicians to recommend cereal pulverization to break down flake size prior to mixing. However, little is known regarding how cereal type and its particle size influence formula thickness. This investigation elucidated these attributes by testing differences in thickening attributes between rice and oatmeal cereals in their off-the-shelf and pulverized formulation.

Method(s): Methods: Similac Advance $(20 \mathrm{kcal} / \mathrm{oz})$ was thickened with graduated volumes $(2,3,4,5$ tsp cereal/2 oz formula) of off-theshelf and pulverized Beech-Nut rice and oatmeal cereal. Pulverization was completed using mortar and pestle. Thickened formulas underwent $30 \mathrm{~min}$ of IDDSI Flow Testing. Differences in dry cereal particle size in off-the-shelf and pulverized formulation were measured using an Olympus-IX73 microscope. Differences in conditions were compared using repeated-measures ANOVA.

Result(s): Results: Oatmeal increased in thickness in off-the-shelf and pulverized formulations throughout the $30 \mathrm{~min}$ testing period $(p=0.01)$, while rice remained relatively constant in thickness. Examination of off-the-shelf rice cereal revealed an average grain radius of $21.9 \mu \mathrm{m}$. Upon thickening there was a stepwise increase in IDDSI thickness level with every additional tsp of cereal added, ranging from thin designation at $2 \mathrm{tsp} / 2 \mathrm{oz}$ formulation to moderately thick at $5 \mathrm{tsp} / 2 \mathrm{oz}$. In contrast, oatmeal cereal had an average grain radius of $225.5 \mu \mathrm{m}$ and generated thicker formula than rice cereal at $3 \mathrm{tsp} / 2 \mathrm{oz}$ (mildly thick) and $4 \mathrm{tsp} / 2 \mathrm{oz}$ (moderately thick) $(p<0.001)$. Pulverization of the cereals prior to mixing resulted in smaller average grain radius for oatmeal $(155.8 \mu \mathrm{m})$, and generated thicker formulations than when the cereals were mixed in their offthe-shelf form $(p=0.000)$.

Conclusions (Including Clinical Relevance): Conclusions: Infant fo,rmula thickness is influenced by cereal type and cereal particle size. Utilization of standard cereal thickening recipes across all cereal types and mixing instructions may have deleterious treatment effects. Relevant Financial Relationships: Abbey Sterkowitz: Has affiliations to disclose;Undergraduate Research Opportunities Program, University of Minnesota-Twin Cities:Grant:Other Activities I Abigail Spoden: Has affiliations to disclose;Undergraduate Research Opportunities Program, University of Minnesota-Twin Cities:Grant:Other Activities | Harrison Frisk: Has affiliations to disclose;University of Minnesota:Salary/Stipend:Employment । Michael Beckstrand: Has affiliations to disclose;University of Minnesota:Salary/Stipend:Employment | Katlyn McGrattan: Has affiliations to disclose;University of Minnesota:Salary/Stipend:Employment;Masonic Children's Hospital:Salary/ Stipend:Employment;Dr. Brown's Medical:Speaking fee:Other Activities;Biogen:Consulting fee:Consulting;Avexis:Consulting fee:Consulting;Biogen,
US-SPN-19-11520:Grant:Other 
Activities;NIH, NICHD, 1R41HD104305-01:Grant:Other Activities;nuBorn Medical:Ownership interest (e.g., stocks, stock options or other ownership interest excluding diversified mutual funds):Ownership;Science Stand:Ownership interest (e.g., stocks, stock options or other ownership interest excluding diversified mutual funds):Ownership.

Relevant Non-financial Relationships: Abbey Sterkowitz: Nothing to Disclose I Abigail Spoden: Nothing to Disclose I Harrison Frisk: Nothing to Disclose I Michael Beckstrand: Nothing to Disclose |
Katlyn McGrattan: Has a Non-Financial Disclosure Affliation;DRS Membership Committee:Professional:Membership;ASHA Pediatric Normal and Disordered Feeding \& Swallowing Topic Committee:Professional:Membership.

Publisher's Note Springer Nature remains neutral with regard to jurisdictional claims in published maps and institutional affiliations. 